\title{
DEVELOPMENT AND CHARACTERIZATION OF FLUORESCENT MANNOSE- AND SIALIC ACID-BASED CHEMICAL PROBES FOR LECTIN DISCOVERY AND ENZYME STUDIES
}

by

\author{
Tasnim Abukar \\ B.Sc., Ryerson University, 2017 \\ A thesis presented to Ryerson University \\ in partial fulfillment of the requirements for the degree of \\ Master of Science in the program of \\ Molecular Science
}

Toronto, Ontario, Canada, 2019

(C) Tasnim Abukar 2019 


\section{AUTHOR'S DECLARATION FOR ELECTRONIC SUBMISSION OF A THESIS}

I hereby declare that I am the sole author of this thesis. This is a true copy of the thesis, including any required final revisions, as accepted by my examiners.

I authorize Ryerson University to lend this thesis to other institutions or individuals for the purpose of scholarly research

I further authorize Ryerson University to reproduce this thesis by photocopying or by other means, in total or in part, at the request of other institutions or individuals for the purpose of scholarly research.

I understand that my thesis may be made electronically available to the public. 


\begin{abstract}
DEVELOPMENT AND CHARACTERIZATION OF FLUORESCENT MANNOSE- AND SIALIC ACID BASED CHEMICAL PROBES FOR LECTIN DISCOVERY AND ENZYME STUDIES
\end{abstract}

Master of Science, 2019

Tasnim Abukar

Molecular Science

Ryerson University

BODIPY (4,4-Difluoro-4-boro-3a,4a-diaza-s-indacene) dyes are relatively nonpolar fluorescent molecules used as biological labeling reagents. The advantages of using BODIPY dyes as tags include their unique photochemical properties, allowing for an easy and sensitive monitoring of target components. This thesis will discuss the synthesis of novel fluorescent "BODIPY"-labeled carbohydrate-based chemical probes and highlight their uses in different biological applications. In chapter one, we have focused on the development of BODIPY-mannose-based chemical probes for the discovery and characterization of mannose-binding lectins on surfaces of Actinobacteria. The synthesis of these probes and their efficiency to detect mannose-binding lectins using different techniques such as fluorescence microscopy and flow cytometry are discussed. In chapter two, we have focused on the chemo-enzymatic synthesis of BODIPY-sialic acid based chemical probes for enzymatic detection of specific glycoconjugates on proteins. The use of these probes as donor substrates to probe the enzymes involved in glycan sialylation are discussed. 


\section{ACKNOWLEDGMENT}

First, I would like to express my sincere gratitude to my supervisors Dr. Warren Wakarchuk and Dr. Bryan Koivisto for taking me as a graduate student and for their continuous support, and guidance throughout my time as a student at Ryerson University. They have been great mentors and it is an honour and privilege to work with them, as they helped me grow as a scientist. I would also like to thank my research committee Dr. Mark Nitz and Dr. Stefania Impellizzeri for their guidance and valuable feedback on my research. I am thankful to Ryerson University and The Department of Chemistry and Biology for this opportunity to study in the molecular science graduate program and learn from the knowledgeable faculty members.

I would also like to thank all my lab colleagues past and present, as well as my friends who have helped me during my graduate study and made my research experience enjoyable and unforgettable.

Last but not least, I would like to thank my entire family, especially my parents Dahaba Nur and Abdirashid Hussein for their unconditional love and support throughout my life. 


\section{TABLE OF CONTENTS}

AUTHOR'S DECLARATION ii

ABSTRACT iii

ACKNOWLEGMENT iv

LIST OF TABLES viii

LIST OF FIGURES viii

LIST OF ABBREVIATIONS xiv

Chapter 1: Development of Mannose-based Fluorescent Probes for Mannose-binding Lectin Discovery

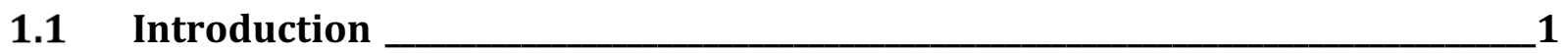

1.1.1 Overview of Lectins____ 1

1.1.2 Detection methods of Lectins and Their Shortcomings___ 4

1.1.3 Small-Molecule Probes ___ 5

1.1.3.1 Recognition Element___ 6

1.1.3.2 Fluorescent tags___ 7

1.1.3.3 Linker __ 9

1.1.4 Carbohydrate-based Probes and its Components____ 11

1.1.5 Project Objectives__ 12

1.1.5.1 Control Mannose-binding Lectin - FimH 15

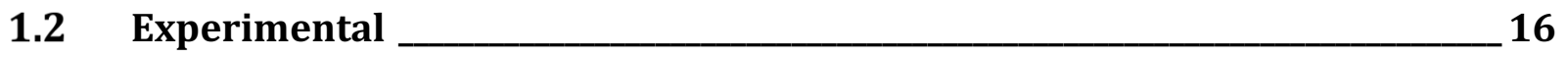

1.2.1 Materials__ 16

1.2.2 General method for compound characterizations ___ 17

1.2.3 Purification technique using C-18 silica columns ___ 17

1.2.4 Purification using preparative glass-backed TLC plates___ 18

1.2.5 Synthesis and Purification of Mannose-based Probes __ 19

1.2.5.1 Synthesis of BODIPY-PEG-Mannose _ 19

1.2.5.2 Synthesis of BODIPY-Mannobiose _ 20 
1.2.5.3 Synthesis of Coumarin-Mannobiose 21

1.2.5.4 Synthesis of BODIPY-PEG-amine __ 22

1.2.6 Growth of Bacteria___ 23

1.2.6.1 Growth conditions for C. fimi and C. flavigina __ 23

1.2.6.2 Growth conditions for FimH E.coli and FimH mutant E.coli __ 23

1.2.7 Flow Cytometry Analysis___ 24

1.2.7.1 Direct \& Inhibition Binding Preparation with PFA fixation 24

1.2.7.2 Direct \& Inhibition Binding Preparation with $\mathrm{NaN}_{3}$ treatment___ 25

1.2.8 Fluorescence Microscopy ___ 26

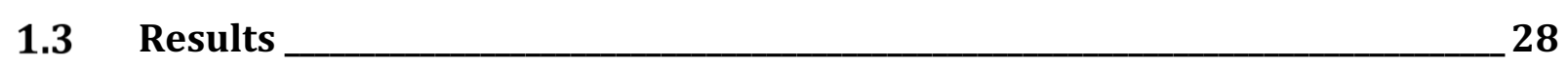

1.3.1 Development of Mannose-based Probes___ 28

1.3.1.1 Synthesis of BODIPY-PEG-Mannose Probes___ 28

1.3.1.2 Synthesis of BODIPY-Mannobiose probe__ 29

1.3.1.3 Synthesis of Coumarin-Mannobiose __ 30

1.3.2 Flow Cytometry Analysis___ 33

1.3.2.1 Direct binding and inhibition binding of mannose probes on FimH E.coli _ 33

1.3.2.2 Direct binding and inhibition binding of mannose probes on C. fimi ___ 41

1.3.2.3 Direct binding and inhibition binding of mannose probes on C. flavigena _ 45

1.3.3 Fluorescence Microscopy ___ 47

1.4 Discussion__ 50

1.4.1 Development of the mannose-based probes __ 50

1.4.2 Functionality of mannose-based probes on FimH E.coli__ 51

1.4.3 Discovery of mannose-binding lectins on C. fimi and C. flavigena ___ 53

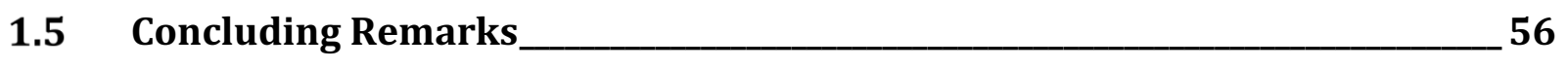

1.5.1 Future Work 56

\section{Chapter 2: $\quad$ Development of Sialic Acid-based Fluorescent Probes for Enzyme Studies}

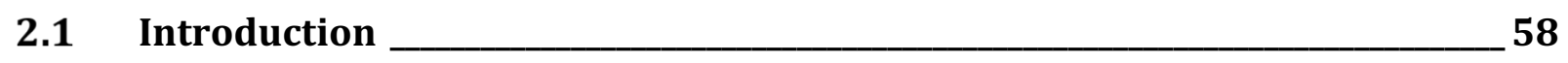

2.1.1 Overview of Sialoglycans___ 58

2.1.2 Types of Sialoglycans _ 59 
2.1.3 Sialyltransferases _ 62

2.1.4 ST3Gal1 vs. ST6Gal1__ 63

2.1.5 Probing Sialylation __ 64

2.1.6 Project objectives__ 66

2.1.6.1 Acceptor Proteins for Modification___ 67

2.2 Experimental __ 68

2.2.1 Materials__ 68

2.2.2 Analysis of compounds by MALDI-TOF mass spectroscopy___ 68

2.2.3 Synthesis and Purification of BODIPY-Sialic acid Probes___ 69

2.2.3.1 Synthesis and Purification of SiaNAz _ 69

2.2.3.2 Synthesis of activated azido-sialic acid-CMP substrates _ 70

2.2.3.3 Purification of azido-sialic acid-CMP substrates__ 70

2.2.3.4 BODIPY-labelling of Neu5Ac-CMP substrates__ 71

2.2.4 Human ST3Gal1 Modification of Interferon $\alpha-2 b \_72$

2.2.5 Human ST6Gal1 Modification of Asialo-A1AT and BuChE _ 72

2.3 Results___ 74

2.3.1 Development of BODIPY-Neu5Ac probes _ 74

2.3.1.1 Synthesis of SiaNAz 74

2.3.1.2 Activation of azido-Neu5Ac-CMP substrates__ 76

2.3.1.3 BODIPY-labelled Neu5Ac-CMP derivatives _ 78

2.3.2 Functional testing of BODIPY-Neu5Ac probes on proteins _ 80

2.3.2.1 Sialylation of IFN $\alpha-2 b$ using Human ST3Gal1 _ 80

2.3.2.2 Sialylation of BuChe and AS- $\alpha 1$ AT Human ST6Gal1___ 82

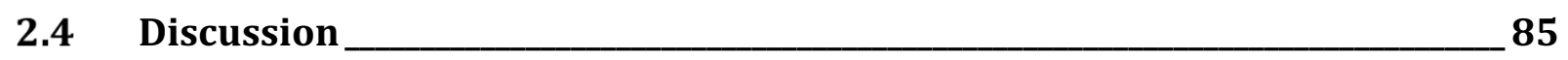

2.5 Concluding Remarks_____ 87

2.5.1 Future Work__ 87

Appendices — 89

References — 93 


\section{LIST OF TABLES}

Table 1: Properties of common bound fluorescent dyes used in biological studies 9

\section{LIST OF FIGURES}

Figure 1: Arrangements of carbohydrate-binding domains (CRDs) of lectins. A) Lectins comprising of just CRDs; B) Lectins consisting of CRDs associated with other functional domains; C) Lectins consisting of CRDs associated with membrane anchors; and D) Lectins consisting of CRDs associated with oligomerization domains. ${ }^{2}$

Figure 2: "Jelly-roll fold" structure of L-type lectins. A) Simple illustration of the "jelly-roll fold" configuration. B) "Jelly-roll fold" structure represented in monomeric concanavalin A (Con A).4, 10

Figure 3: Schematic representation of a small-molecule biological probe. 6

Figure 4: Core structures of common bound fluorescent dyes. 8

Figure 5: General reaction scheme for copper-catalyzed azide-alkyne cycloaddition (CuAAC).

Figure 6: General conditions of a succinimidyl ester reaction 11

Figure 7: Chemical structures of proposed BODIPY-mannose-based probes. Recognition element (red); linker (purple); and fluorescent tag (green). 14

Figure 8: Synthesis of BODIPY-PEG-Mannose Probes. A) Chemical structures of the starting materials used in the CuAAc reaction. B) TLC analysis of BODIPY-PEG-mannose (1.4) synthesis. Lane 1: starting material, BODIPY-alkyne; Lanes 2: CuAAc reaction mixture, time 
point: 2h. C) TLC analysis of Planar-BODIPY-PEG-mannose (1.5) synthesis. Lane 1: starting material, planar BODIPY-alkyne; Lanes 2: CuAAc reaction mixture, time point: 10h. 29

Figure 9: Synthesis of BODIPY-Mannobiose. A) Succinimidyl ester reaction scheme of BODIPY-mannobiose and B) TLC analysis of BODIPY-mannobiose synthesis. Lane 1: starting material, BODIPY-NHS; Lanes 2: reaction mixture, time point: $3 \mathrm{~h}$; Lane 3: purified BODIPY-mannobiose fraction. 30

Figure 10: Succinimidyl ester reaction scheme of coumarin-mannobiose 31

Figure 11: Synthesis of Coumarin-Mannobiose. A) Purification of coumarin-mannobiose by size-exclusion chromatography and B) TLC analysis of coumarin-mannobiose reaction mixture and fractions from purification. Lane 1: starting material, Coumarin-NHS; Lanes 2: reaction mixture, time point: $22 \mathrm{~h}$; Lanes 3-5: fractions from purification. Lane 3: fraction a (purified coumarin-mannobiose); Lane 4: fraction b; and Lane 5: fraction c 32

Figure 12: Investigating the binding specificity of BODIPY-PEG-Mannose probe for FimH lectins by flow cytometry. Direct binding analyses of probes binding to A) FimH E.coli cells $\left(\mathrm{OD}_{600}=1\right)$ and B) FimH mutant E.coli cells $\left(\mathrm{OD}_{600}=1\right)$. Negative control, probe absent (red); cells incubated with $25 \mu \mathrm{M}$ BODIPY-PEG-Mannose amine (orange); and cells incubated with $25 \mu \mathrm{M}$ BODIPY-PEG-Mannose probe (blue). MFI: median fluorescence intensity

Figure 13: Inhibition binding studies to investigate the selectivity of BODIPY-PEGMannose probe binding to FimH E.coli cells by flow cytometry. Negative control, probe absent (red). Non-specific binding of $25 \mu \mathrm{M}$ control BODIPY-PEG-amine probe to FimH E.coli cells (green). Selective binding of $25 \mu \mathrm{M}$ BODIPY-PEG-mannose probe to FimH E.coli cells (orange). Inhibition of this selective binding by the competitive ligand: 4-nitrophenyl $\alpha$-D-mannopyranoside (blue). Analyses were all done with FimH E.coli cells at $\mathrm{OD}_{600}=1$. For each sample the analysis of 100,000 events were done. MFI: median fluorescence intensity. 36 
Figure 14: Inhibition binding studies to investigate the non-specific binding of BODIPYPEG-Mannose probe to FimH mutant E.coli cells by flow cytometry. Negative control, probe absent (red). Non-specific binding of $25 \mu \mathrm{M}$ control BODIPY-PEG-amine probe to FimH mutant E.coli cells (blue). Non-specific binding of $25 \mu \mathrm{M}$ BODIPY-PEG-mannose probe to FimH mutant E.coli cells (orange). Inhibition studies with competitive ligand: 4-nitrophenyl $\alpha$-D-mannopyranoside (green). Analyses were all done with FimH mutant E.coli cells at $\mathrm{OD}_{600}=1$. For each sample the analysis of 100,000 events were done. MFI: median fluorescence intensity.

Figure 15: Binding comparison of BODIPY-PEG-Mannose and BODIPY-Mannobiose probes to FimH E.coli by flow cytometry. Negative control, probe absent (red). Binding of $0.1 \mathrm{mM}$ BODIPY-PEG-mannose probe to FimH E.coli cells (blue). Binding of $0.1 \mathrm{mM}$ BODIPYmannobiose probe to FimH E.coli cells (orange). Analyses were all done with FimH E.coli cells at $\mathrm{OD}_{600}=1$. For each sample the analysis of 100,000 events were done. MFI: median fluorescence intensity. 38

Figure 16: Chemical structure of BODIPY-Mannobiose and Coumarin-Mannobiose 39

Figure 17: Inhibition binding studies to investigate the selectivity of BODIPY-Mannobiose probe binding to FimH E.coli cells by flow cytometry. Negative control, probe absent (red). Non-specific binding of $0.1 \mu \mathrm{M}$ control BODIPY-PEG-amine probe to FimH E.coli cells (blue). Selective binding of $0.1 \mu \mathrm{M}$ BODIPY-PEG-mannose probe to FimH E.coli cells (orange). Inhibition of this selective binding by the competitive ligand: coumarin-mannobiose (green). Analyses were all done with FimH E.coli cells at $\mathrm{OD}_{600}=1$. For each sample the analysis of 100,000 events were done. MFI: median fluorescence intensity. 40

Figure 18: Binding performance of BODIPY-PEG-amine and BODIPY-PEG-Mannose probe to $C$.fimi cells by flow cytometry. The following $\mathrm{OD}_{600}$ values were tested: $\left.\mathrm{A}\right) \mathrm{OD}_{600} \approx 1.0, \mathrm{~B}$ ) $\mathrm{OD}_{600} \approx 0.6$ and $\mathrm{C}$ ) $\mathrm{OD}_{600} \approx 0.3$. Negative control, probe absent (red). Non-specific binding of $25 \mu \mathrm{M}$ control BODIPY-PEG-amine probe to C.fimi cells (blue). Direct binding studies of 25 $\mu \mathrm{M}$ BODIPY-PEG-mannose probe binding to $C$.fimi cells. For each sample the analysis of 100,000 events were done. MFI: median fluorescence intensity 
Figure 19: Investigating the selectivity of BODIPY-PEG-Mannose probe binding to C.fimi cells by flow cytometry. Negative control, probe absent (red). Non-specific binding of 0.1 $\mathrm{mM}$ control BODIPY-PEG-amine probe to $C$.fimi cells (blue). Selective binding of $0.1 \mathrm{mM}$ BODIPY-PEG-mannose probe to C.fimi cells (orange). Inhibition of this selective binding by the competitive ligand: 4-nitrophenyl $\alpha$-D-mannopyranoside (green). For each sample the analysis of 100,000 events were done. MFI: median fluorescence intensity. 43

Figure 20: Binding comparison of BODIPY-PEG-Mannose and BODIPY-Mannobiose probes to $C$.fimi by flow cytometry. Negative control, probe absent (red). Non-specific binding of $0.1 \mathrm{mM}$ control BODIPY-PEG-amine probe to C.fimi cells (blue). Binding of 0.1 mM BODIPYPEG-mannose probe to C.fimi cells (orange). Binding of $0.1 \mathrm{mM}$ BODIPY-mannobiose probe to $C$.fimi cells (green). Analyses were all done with cells at $\mathrm{OD}_{600}=0.231$. For each sample the analysis of 100,000 events were done. MFI: median fluorescence intensity.

Figure 21: Investigating the selectivity of BODIPY-PEG-Mannose probe binding to C.flavigena cells by flow cytometry. Negative control, probe absent (red). Non-specific binding of $0.1 \mathrm{mM}$ control BODIPY-PEG-amine probe to C.flavigena cells (blue). Selective binding of $0.1 \mathrm{mM}$ BODIPY-PEG-mannose probe to C.flavigena cells (orange). Inhibition of this selective binding by the competitive ligand: 4-nitrophenyl $\alpha$-D-mannopyranoside (green). For each sample the analysis of 100,000 events were done. MFI: median fluorescence intensity. 46

Figure 22: Binding comparison of BODIPY-PEG-Mannose and BODIPY-Mannobiose probes to C.flavigena by flow cytometry. Negative control, probe absent (red). Non-specific binding of $0.1 \mathrm{mM}$ control BODIPY-PEG-amine probe to C.flavigena cells (blue). Binding of $0.1 \mathrm{mM}$ BODIPY-PEG-mannose probe to C.flavigena cells (orange). Binding of 0.1 mM BODIPYmannobiose probe to C.flavigena cells (green). Analyses were all done with cells at $0_{600}=$ 0.234. For each sample the analysis of 100,000 events were done. MFI: median fluorescence intensity. 47

Figure 23: FimH E.coli Microscopy images for co-localization investigation with BODIPYPEG-mannose probe. FimH E.coli (NRG857c) at $\mathrm{OD}_{600}=1.00$ incubated with $6 \times 10^{-3} \mathrm{mM}$ of 
BODIPY-PEG-mannose probe. Channels: 1) Bright field, 2) DAPI, 3) BODIPY and 4) Merged.

Figure 24: C.fimi Microscopy images for co-localization investigation. A) Cells incubated with $25 \times 10^{-2} \mathrm{mM}$ of BODIPY-PEG-mannose probe. B) Cells incubated with $25 \times 10^{-2} \mathrm{mM}$ of BODIPY-mannobiose probe. Studies were done with cells at $\mathrm{OD}_{600}=0.231$. Channels: 1 ) Bright field, 2) DAPI, 3) BODIPY and 4) Merged. 49

Figure 25: The general chemical structure of sialic acid. Various structural of modifications can occur at the R positions. 59

Figure 26: Common core carbohydrate sequences of N-linked glycan structures. ${ }^{40}$ 61

Figure 27: Biosynthesis of O-glycan chains. Illustration of Core-1 (Tn antigen), Core-2 ( $\mathrm{T}$ antigen) and Core 3 subtype formations. Modifications of $\mathrm{Tn}$ antigen and $\mathrm{T}$ antigens by ST6GalNAc1-2 and ST3Gal1 generating sialyl-Tn antigen and sialyl-T antigens. ${ }^{48}$ 64

Figure 28: Chemical structures of C-5 and C-9 azido modified sialic acids. 67

Figure 29: Synthesis of SiaNAz. A) Aldol condensation reaction scheme of siaNAz and B) TLC analysis of the siaNAz synthesis. Lane 1: starting material, ManNAz; Lanes 2: aldol condensation reaction, time point: $72 \mathrm{~h}$; Lane 3: starting material, sodium pyruvate.

Figure 30: Activation of azido-Neu5Ac substrates via CMP- $\beta$-sialic acid synthetase (NSY05) A) Reaction scheme of the azido-Neu5Ac-CMP substrates. C-5 modified Neu5Ac $\left(R_{1}=\right.$ $\left.\mathrm{N}_{3}, \mathrm{R}_{2}=\mathrm{OH}\right)$ and C-9 modified Neu5Ac $\left(\mathrm{R}_{1}=\mathrm{H}, \mathrm{R}_{2}=\mathrm{N}_{3}\right]$. B) TLC analysis of CMP-conjugate synthesis. I) Synthesis of siaNAz-CMP and; II) Synthesis of 9-azido-Neu5Ac-CMP. Lane 1: starting material, CTP; Lane 2: Negative control, NSY-05 absent; Lanes 3-4, NSY-05 present, time points: $2 \mathrm{~h}$ and $3 \mathrm{~h}$.

Figure 31: Activation of BODIPY-9-Neu5Ac via CMP- $\beta$-sialic acid synthetase (NSY-05). A) Reaction scheme of the BODIPY-9-Neu5Ac-CMP substrates. B) TLC analysis of BODIPY-9- 
Neu5Ac-CMP synthesis. Lane 1: Negative control, NSY-05 absent. Lanes 2: NSY-05 present, time point: $3 \mathrm{~h}$ 78

Figure 32: Synthesis of BODIPY-labelled Neu5Ac-CMP derivatives using CuAAc reaction. A) BODIPY-9-Neu5Ac-CMP: I) chemical structure and; II) TLC analysis of BODIPY-9-Neu5AcCMP synthesis, Lane 1: starting material, BODIPY-alkyne; and Lane 2: CuAAc reaction mixture, time point 1hr. B) BODIPY-5-Neu5Ac-CMP: I) chemical structure and; II) TLC analysis of BODIPY-5-Neu5Ac-CMP synthesis, Lane 1: starting material, BODIPY-alkyne; and Lane 2: CuAAc reaction, time point $1 \mathrm{hr}$.

Figure 33: Comparison of BODIPY-Neu5Ac-CMP probes in the modification of IFN $\alpha-2 b$ using human ST3Gal1. A) SDS-PAGE comparison of the probes (0.05mM): I) BODIPY-9Neu5Ac-CMP and ii) BODIPY-5-Neu5Ac-CMP. B) Densitometric analysis: a quantitative study to determine which probe is efficiently transferred onto $1 \mathrm{mg} / \mathrm{mL}$ protein using 0.1 $\mathrm{mg} / \mathrm{mL}$ ST3Gal1. Coomassie blue staining (CBB). Lane 1: Negative control; ST3Gal1 absent. Lanes 2-7: ST3Gal1 present, time points 15, 30, 60, 120, 180 min and overnight. 81

Figure 34: Comparison of BODIPY-Neu5Ac-CMP probes in the modification of BuChE using human ST6Gal1. A) SDS-PAGE comparison of the probes (0.1mM) BODIPY-9-Neu5Ac-CMP and BODIPY-5-Neu5Ac-CMP. B) Densitometric analysis: a quantitative study to determine which probe is efficiently transferred onto $2 \mathrm{mg} / \mathrm{mL}$ protein using $0.1 \mathrm{mg} / \mathrm{mL}$ ST6Gal1. Coomassie blue staining (CBB). Lane 1: Negative control; ST6Gal1 absent. Lanes 2-7: ST6Gal1 present, time points 0, 30, 60, 120, 240 min and overnight. 83

Figure 35: Comparison of BODIPY-Neu5Ac-CMP probes in the modification of AS- $\alpha 1 \mathrm{AT}$ using human ST6Gal1. A) SDS-PAGE comparison of the probes (0.1 mM) BODIPY-9-Neu5AcCMP and BODIPY-5-Neu5Ac-CMP. B) Densitometric analysis: a quantitative study to determine which probe is efficiently transferred onto $2 \mathrm{mg} / \mathrm{mL}$ protein using $0.1 \mathrm{mg} / \mathrm{mL}$ ST6Gal1. Coomassie blue staining (CBB). Lane 1: Negative control; ST6Gal1 absent. Lanes 2-7: ST6Gal1 present, time points 0, 30, 60, 120, 240 min and overnight. 84 


\section{LIST OF ABBREVIATIONS}

\begin{tabular}{|c|c|}
\hline 9-AA & 9-aminoacridine \\
\hline Asialo- $\alpha 1 A T$ & Asialo-alpha 1 anti-trypsin \\
\hline Asn & Asparagine \\
\hline$\beta$-GIcNAc & $\beta$-N-acetylglucosamine \\
\hline BODIPY & 4,4 -Difluoro-4-boro-3a, 4a-diaza-s-indacene \\
\hline BuChE & Butyrylcholinesterase \\
\hline C.fimi & Cellulomonas fimi \\
\hline C.flavigena & Cellulomonas flavigena \\
\hline CMP & Cytidine 5'-monophosphate \\
\hline CRD & Carbohydrate-recognition domains \\
\hline CTP & Cytidine 5'-triphosphate \\
\hline CuAAC & Copper-Catalyzed Azide-Alkyne Cycloaddition \\
\hline DAPI & 4, 6-diamidino-2-phenylindole \\
\hline DMF & Dimethylformamide \\
\hline E.coli & Escherichia coli \\
\hline EtOAc & Ethyl acetate \\
\hline ESI & Electrospray ionization \\
\hline Gal & Galactose \\
\hline GlcNAc & $\mathrm{N}$-acetylglucosamine \\
\hline HOAC & Acetic acid \\
\hline IFN $\alpha-2 b$ & Interferon alpha- $2 b$ \\
\hline
\end{tabular}




\begin{tabular}{|c|c|}
\hline KDN & 2-keto-3-deoxynononic acid \\
\hline $\mathbf{m} / \mathbf{z}$ & Mass to charge ratio \\
\hline MALDI-TOF & Matrix assisted laser desorption/ionization - Time of flight \\
\hline Man & Mannose \\
\hline $\mathrm{NaN}_{3}$ & Sodium azide \\
\hline Neu5Ac & $\mathrm{N}$-acetylneuraminic acid \\
\hline NeuGc & $\mathrm{N}$-glycolylneuraminic acid \\
\hline NHS & N-hydroxysuccinimide \\
\hline $\mathrm{NH}_{4} \mathrm{OH}$ & Ammonium hydroxide \\
\hline NMR & Nuclear magnetic resonance \\
\hline NSY-05 & Neisseria meningitides CMP-sialic acid synthetase \\
\hline $\mathrm{OD}_{600}$ & Optical density at $\lambda 600 \mathrm{~nm}$ \\
\hline PAA & Polyacrylamide \\
\hline PBS & Phosphate-buffered saline \\
\hline PEG & Polyethylene glycol \\
\hline PFA & Paraformaldehyde \\
\hline PPE & Poly ( $\rho$-phenylene ethynylene) \\
\hline $\mathbf{R}_{\mathbf{f}}$ & Retention factor \\
\hline Ser & Serine \\
\hline ST3Gal & ST3 $\beta$-galactoside $\alpha$-2,3-sialyltransferases \\
\hline ST6Gal & ST6 $\beta$-galactoside $\alpha$-2,6-sialyltransferase \\
\hline THPTA & Tris 3-hydroxypropyltriazolylmethylamine \\
\hline Thr & Threonine \\
\hline
\end{tabular}




\section{Chapter 1: Development of Mannose-based Fluorescent Probes for Mannose-binding Lectin Discovery}

\subsection{Introduction}

\subsubsection{Overview of Lectins}

Lectins are carbohydrate-binding proteins that are ubiquitous in nature and have the common ability to recognize and bind to specific carbohydrate structural epitopes without altering them. ${ }^{1}$ The distinctive carbohydrates decorated on the surfaces of cells make them targets for lectins. Lectins are typically comprised of two or more binding sites specific for carbohydrate molecules, referred to as the carbohydrate-recognition domains (CRDs). ${ }^{2}$ These domains mediate lectin activity and can exist in four central arrangements. CRDs alone can constitute the entire lectin (Figure 1A), which results in its function being reliant on multivalency. ${ }^{2}$ This arrangement explains the ability of specific plant lectins to cluster glycoproteins on cell surfaces, which results in their ability to agglutinate cells. ${ }^{2}$ CRDs associated with extra functional domains permit the protein to carry out several biological functions including enzymatic reactions, ligand-binding and anchoring proteins to cell membranes (Figure 1B). ${ }^{2}$ CRDs associated with membrane anchors connected to the cytoplasmic regions facilitate transfer of information (Figure 1C). ${ }^{2}$ CRDs associated with oligomerization domains enable multivalent binding to increase the binding strength and direct the geometric position of binding sites. ${ }^{2}$ (Figure 1D). 


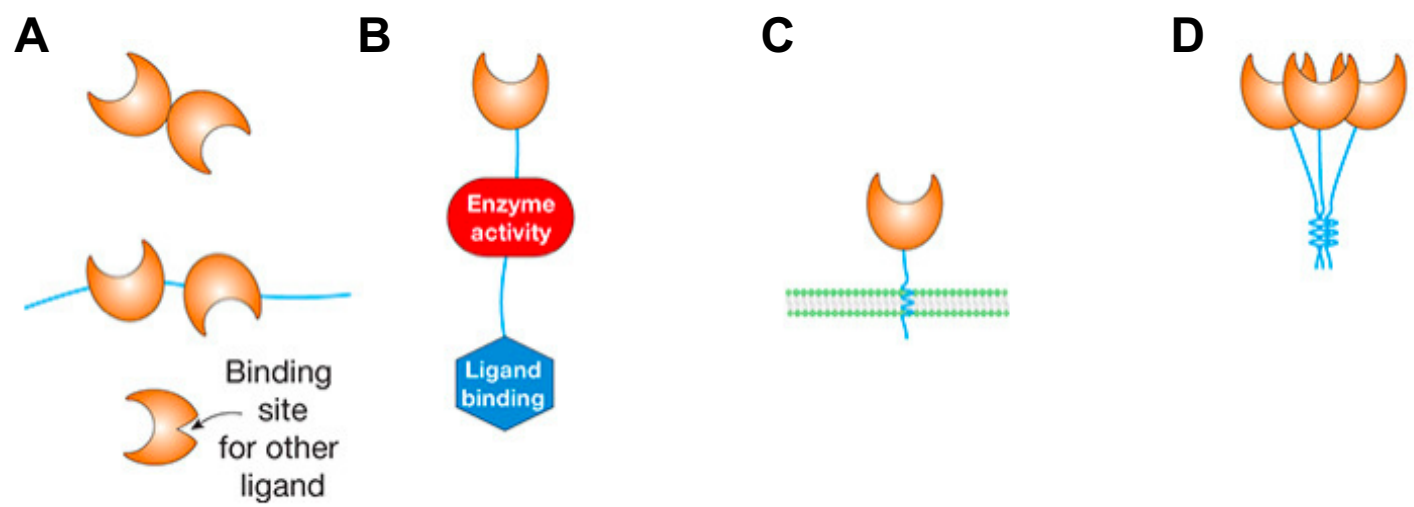

Figure 1: Arrangements of carbohydrate-binding domains (CRDs) of lectins. A) Lectins comprising of just CRDs; B) Lectins consisting of CRDs associated with other functional domains; C) Lectins consisting of CRDs associated with membrane anchors; and D) Lectins consisting of CRDs associated with oligomerization domains. ${ }^{2}$

Besides the arrangements of CRDs, lectins are also classified by their structural homology and evolutionary similarities. Their structure can range from flat surfaces to deeply buried pockets depending on the type of lectin. ${ }^{3}$ Typical structural folds of lectin CRDs tabulated from numerous crystallographic and spectroscopic studies are associated to specific lectin groups. For example, L-type lectins, which are a large group of lectins found in plants and animals, have a common tertiary structural motif known as the "jelly-roll fold" illustrated in Figure 2. 2,4 
A

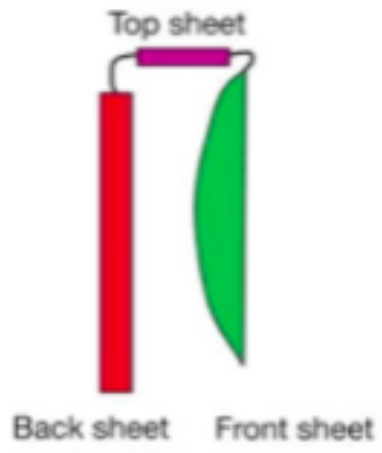

B

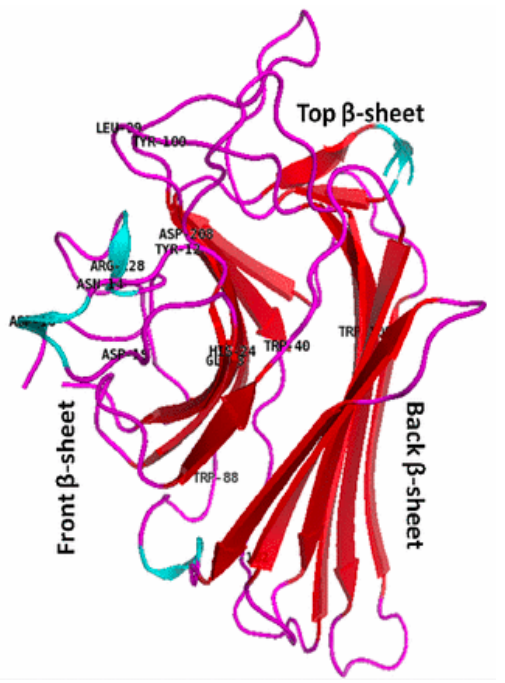

Figure 2: "Jelly-roll fold" structure of L-type lectins. A) Simple illustration of the "jellyroll fold" configuration. B) "Jelly-roll fold" structure represented in monomeric concanavalin A (Con A).4,10

At the CRD, lectin-carbohydrate binding is reversible and occurs through the following weak interactions: hydrogen bonding, van der Waals, hydrophobic interactions and metal ions (i.e. $\mathrm{Ca}^{2+}, \mathrm{Mg}^{2+}$ and $\mathrm{Mn}^{2+}$ ). Lectin-carbohydrate interactions lead to conformational changes that mediate specific interactions in living organisms like cell-cell contact, cell-cell communication, cell signaling, and cell development. 5, 6 These interactions also play an essential role in various pathological processes such as infection, cancer cell metastasis, and inflammation. ${ }^{6}$ For instance, galectins a family of lectins with a similar binding affinity for $\beta$ galactoside sugars are involved in immune and inflammatory responses. ${ }^{4}$ Hepatic lectins, a group of membrane receptor proteins which specifically bind $\beta$ - $\mathrm{N}$ acetylglucosamine ( $\beta$-GlcNAc) moieties have been reported to be capable of mediating endocytosis. ${ }^{7}$ Cell surface glycoproteins bearing terminal mannoside 
residues can act as high affinity ligands for different fimbriated pathogens, enabling pathogen-host recognition. ${ }^{8}$

\subsubsection{Detection methods of Lectins and Their Shortcomings}

As lectins play an important role in a number of diverse biological processes, the search for the development of effective techniques to probe them are continuing. An essential aspect of investigating their biological functions is the identification and evaluation of their quantity in cells. Current methods that exist are practical in detecting lectins though they are accompanied with a few shortcomings. For instance, one method is a carbohydrate affinity chromatography, which involves the use of carbohydrate ligands immobilized to a solid support allowing for the separation of lectins based on their specific interaction with the solid support. The disadvantages of this technique is that there is no definitive proof that all the lectins have eluted from the column or even bound to the solid support. ${ }^{9}$ Another method is a hemagglutination inhibition assays, which determines the specificity of lectins by measuring the amount of carbohydrates required to prevent the agglutination of red blood cells. ${ }^{10}$ Although, this technique is effective at determining the specificity of lectins, the disadvantages is that they do require the use of red blood cells and large amounts of lectin source. ${ }^{10}$ Surface plasmon resonance (SPR) and quartz crystal microbalance (QCM) are methods that have the common ability to detect changes in mass upon lectin-carbohydrate binding. ${ }^{11}$ In both of these methods, the carbohydrate ligand is immobilized onto the surface of a sensor chip and subsequently the lectin solution is flowed over the chip surface, allowing for 
interaction to occur. ${ }^{11,12} \mathrm{~A}$ few disadvantages of these methods are that it cannot easily differentiate specific and non-specific interactions, there are difficulties in the detection of low molecular weight compounds, and in obtaining uniformly functionalized surfaces when using sensor chips. ${ }^{11,}{ }^{12}$ Moreover, current in vitro protein studies do not always adequately elucidate their biological functions. ${ }^{14}$ For this reason, the development of nondestructive analysis tools for use in biological research is highly necessary.

\subsubsection{Small-Molecule Probes}

Contrary to the physical methods described above, small-molecule probes also referred to as molecular probes represent a nondestructive method that has widely been used in biological applications. ${ }^{13,14}$ These probes are tags that have been used to visualize, characterize and quantify biological processes occurring in living systems. ${ }^{14}$ Generally, a chemical probe is comprised of three main structural components: i) the recognition element; ii) tag moiety and; iii) the linker illustrated in Figure 3. ${ }^{27}$ The recognition element in the probe design permits specific interaction with a target. Several types of targeting ligands can be considered for the recognition element like carbohydrates, peptides, proteins, antibody, nucleic acids, nanoparticles and other small molecules. In addition to targeting, the selection of an optimal tag moiety is also important in probe design, as it is the component that

produces signal for imaging purposes. ${ }^{27}$ The tag moiety can range from being a radionuclides (positron-emission tomography), magnetic molecules (magnetic 
resonance imaging), microbubbles (ultrasound), bioluminescence or fluorescent molecules. ${ }^{27}$ Photochemical properties, physical and chemical properties are all key considerations when choosing an ideal tag. For instance, since small molecule biological probes are relatively low mass, the attachment of large dyes can substantially impact the biological function of the recognition element i.e. cell permeability, steric hindrance, hydrophobicity, and non-specific binding properties. The final component of the probe, which is the linker, connects the recognition element with the dye, minimizing the interaction between them. ${ }^{15,27}$ Varying the linker can influence the overall property of the probe such that it improves solubility, flexibility, cell permeability etc.

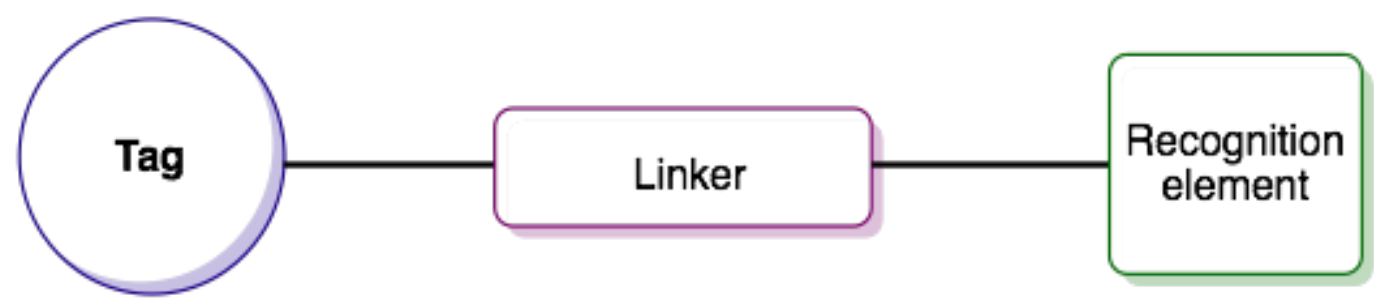

Figure 3: Schematic representation of a small-molecule biological probe.

\subsubsection{Recognition Element}

The recognition element is the key aspect of specificity for small-molecule biological probe technologies. In the design of carbohydrate-based probes for lectin studies, the recognition element must comprise of a specific carbohydrate ligand, which would interact with the target lectins. This carbohydrate ligand can range from monosaccharides, oligosaccharides and polysaccharides, depending on the desired mode of action. It has been reported that carbohydrate-lectin interactions typically 
occur with low binding affinities, though it has been shown that multivalency significantly enhances binding. ${ }^{17,18,19}$ Binding affinities of divalent and trivalent carbohydrate ligands were observed to being up to $10^{3}$ - and $10^{6}$ - fold higher, respectively, than monovalent carbohydrate ligands. ${ }^{17}$ Lee et al., (2006) investigated monovalent and trivalent carbohydrate probes that consisted of a photoreactive benzophenone group, which upon irradiation covalently bound weakly bound lectins. ${ }^{18}$ Within $30 \mathrm{~min}$ of irradiation, strong binding of probes to lectins were observed; however greater binding affinities were seen with the trivalent carbohydrate probes ${ }^{18}$. Similar results were also seen with the carbohydrate-functionalized poly ( $\rho$-phenylene ethynylene) (PPE) probe developed by Disney et al., (2004), where it was displayed that multivalent interactions greatly enhanced the binding affinity and selectivity of the probe to FimH E.coli. ${ }^{19}$

\subsubsection{Fluorescent tags}

Among the number of available methods of detection, fluorescence detection has several distinct advantages, being highly sensitive to sample concentration, less sensitive to instrument variability and allowing for an easy and sensitive monitoring of target components and even their interactions in vivo. Assessment of fluorescence signals is one of the most sensitive methods amongst other analytical techniques. ${ }^{20}$ In order to be used for biological applications the fluorescent dye component should exhibit the following desirable properties, such as; high molar extinction coefficients, low toxicity, high quantum yield $\left(\Phi_{\mathrm{F}}\right)$, large Stokes shift, long 
fluorescence lifetime $\left(\tau_{F}\right)$, and photostability. ${ }^{62}$ Fluorescent dyes used for biological labelling can be categorized with regards to the labelling modalities between fluorescent dye and the labelled biomolecule. ${ }^{21}$ For instance, the fluorescent dye can be bound to the biomolecule via an active group (i.e. azide, alkyne or NHSester); or it can also be inserted into the DNA structure of an organism via transfection (i.e. GFP or RFP) or transiently expressed i.e. GFP-fusion proteins from plasmids. ${ }^{21}$

Among the numerous types of fluorescent dyes used in biological applications, BODIPY (4,4 -Difluoro-4-boro-3a,4a-diaza-s-indacene) dyes show significant promise for this body of work (Figure 4 and Table 1). Being a photostable substitute for fluorescein, it is strongly absorbing and emits relatively sharp fluorescence maxima in the visible and near infrared region with high quantum yields up to $\Phi_{\mathrm{F}}=0.97 .{ }^{22,23,24}$ Due to its relative insensitivity to the $\mathrm{pH}$, solvent and polarity of its environment it makes a preferred choice for the labelling of biomolecules. ${ }^{25}$ Structural modifications of these dyes allow the tuning of their fluorescence characteristics as well as easy labelling to various biomolecules.

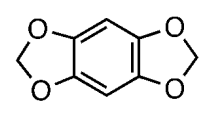

DBD

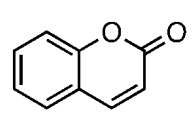

Coumarin

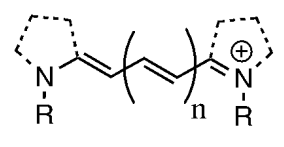

Cyanine

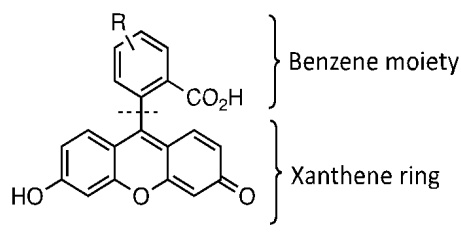

Fluorescein

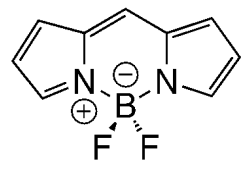

BODIPY

Figure 4: Core structures of common bound fluorescent dyes. 


\begin{tabular}{|c|c|c|c|c|c|c|}
\hline $\begin{array}{l}\text { Fluorescent } \\
\text { Dyes }\end{array}$ & $\begin{array}{c}\text { Absorbance } \\
\lambda_{\text {abs }}(\mathrm{nm})\end{array}$ & $\begin{array}{l}\text { Emission } \\
\lambda_{\text {em }}(\mathrm{nm})\end{array}$ & $\begin{array}{l}\text { Quantum } \\
\text { yield }\left(\Phi_{F}\right)\end{array}$ & Pros & Cons & Reference \\
\hline DBD & $420-440$ & $520-630$ & $0.12-0.57$ & - Large stokes shift & - Photo-bleaching & $20,32,61$ \\
\hline Coumarins & $350-400$ & $430-450$ & $\sim 0.63$ & $\begin{array}{c}\text { - Good water } \\
\text { solubility }\end{array}$ & $\begin{array}{l}\text { - Sensitive to } \mathrm{pH} \\
\text { - Not as bright } \\
\text { - Autofluorescence }\end{array}$ & $68,69,70$ \\
\hline Cyanines & $548-684$ & $570-710$ & $\sim 0.15-0.3$ & - Large stokes shift & $\begin{array}{c}\text { - Sensitive to solvent } \\
\text { changes } \\
\text { - Not as bright }\end{array}$ & $71,72,73$ \\
\hline Fluoresceins & $\sim 492$ & $\sim 515$ & $\sim 0.6-0.78$ & $\begin{array}{c}\text { - Good water } \\
\text { solubility }\end{array}$ & - $\mathrm{pH}$ sensitive & $\begin{array}{c}74,75,76, \\
77\end{array}$ \\
\hline BODIPY & $502-504$ & $509-512$ & $\sim 0.80-0.97$ & $\begin{array}{l}\text { - Insensitive to } \mathrm{pH} \\
\text { - Very bright } \\
\text { - Great photostability }\end{array}$ & Low stokes shift & $\begin{array}{c}22,23,24, \\
25\end{array}$ \\
\hline
\end{tabular}

Table 1: Properties of common bound fluorescent dyes used in biological studies

\subsubsection{Linker}

The overall property of the carbohydrate-based probes can also be optimized in the linker portion, as it joins the recognition element and the tag moiety. The linker component can be varied to control the overall size and property of the probe. However, it should minimize the interactions between the recognition element and the fluorescent tag so that there is no interference between their separate and significant functions. ${ }^{26}$ Therefore, the linker length is an essential parameter as it can influence the functionality of the probe, i.e. allow simultaneous binding of recognition elements to lectins without creating unfavourable enthalpy strains. ${ }^{3} \mathrm{~A}$ really short spacer can result in steric hindrance, whereas, a very long spacer can increase the risk of non-specific binding. Linkers used in carbohydrate-based probes can provide an overall hydrophilic or hydrophobic property, i.e. improving permeability and solubility. For instance, polyethylene glycol (PEG) linkers, are 
biologically inert and nonionic molecules consisting of repeated oxyethylene (-CH2$\mathrm{CH} 2-\mathrm{O}-$ ) subunits that have a molecular weight ranging from $200 \mathrm{~g} \mathrm{~mol}^{-1}$ to $40,000,000 \mathrm{~g} \mathrm{~mol}^{-1} .{ }^{27}$ The popularity of these linkers are primarily due to their ability to enhance the aqueous solubility of hydrophobic molecules, which has previously been demonstrated by Bader et al., (2016) with his developed DBD-PEGmannose probe. ${ }^{28,29}$ Polyacrylamide (PAA) carriers can also be selected as linkers particularly for their hydrophilic and flexibility properties seen in fluorescein-PAA carbohydrate probes developed by Galanina et al., (2001). ${ }^{16}$ These PAA carriers have also exhibited no interference with binding of probe to cellular lectins. ${ }^{16}$ Furthermore, in some cases the linker component can support the recognition element by covalently binding weakly bound lectins to the probe via UV illumination, shown by Lauc et al., (2000), with his developed photoaffinity glycoprobes. ${ }^{30}$

For the development of mannose-based probes, bound fluorescent dyes are the choice of tag moiety due to the ease of conjugation to the probe. Perhaps some of the best linking methods that can be employed for the conjugation of the dye to the probe includes the copper-catalyzed azide-alkyne cycloaddition (CuAAc) reaction or succinimidyl ester reaction, due to their simple reaction conditions, stereospecificity, production of high yields and minimal byproducts that can be simply removed. CuAAc reactions involve the use of a copper (I) catalyst to 'click' a terminal alkyne group to an azide group forming a stable disubstituted triazole linked product illustrated in Figure 5. Succinimidyl ester reaction involves an 
amine-reactive group such as NHS esters reacting with primary amines under alkaline conditions to form a stable amide linked product as illustrated in Figure 6.

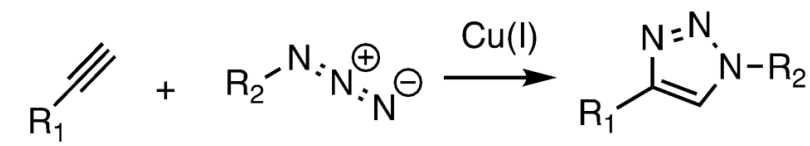

$\begin{array}{lll}\text { Alkyne } & \text { Azide } & \text { Triazole }\end{array}$

Figure 5: General reaction scheme for copper-catalyzed azide-alkyne cycloaddition (CUAAC).

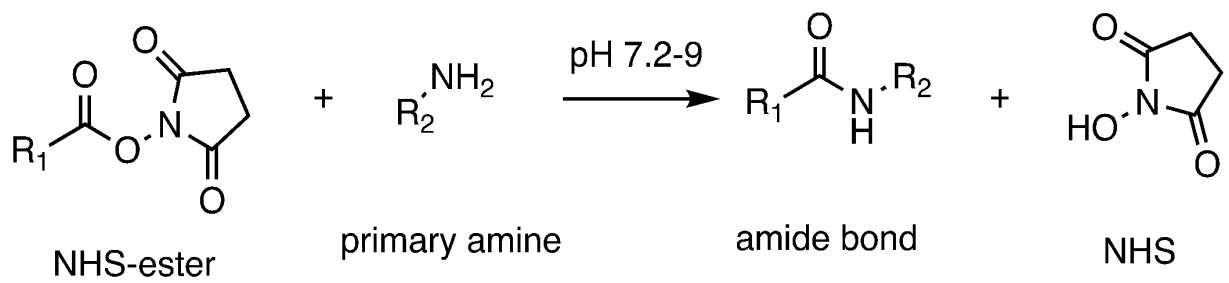

Figure 6: General conditions of a succinimidyl ester reaction.

\subsubsection{Carbohydrate-based Probes and its Components}

Research has shown that the main advantage of using carbohydrate-based chemical probes is that it allows for the detection and identification of new lectins possessing identical or similar carbohydrate-binding properties, even if their sequence homology is unknown. ${ }^{16}$ Carbohydrate-based chemical probes are small molecules that must consist of a carbohydrate moiety as the recognition element for the specific interaction with the target lectin. Whereas, the tag and linker components can vary, depending on the desired molecular property and detection method. Development of effective carbohydrate probe design should provide minimal non- 
specific binding with cellular components. Thus, the tag and linker components must insignificantly affect the specific carbohydrate-lectin interaction.

To date, a few carbohydrate-based chemical probes have been developed to study carbohydrate-lectin interactions. These probes have consisted of recognition elements including monovalent and multivalent carbohydrate ligands; tags ranging from phosphorescent and fluorescent dyes; and linkers varied by size, length, rigidity, and hydrophilic and hydrophobic properties. Although the majority of the carbohydrate-based chemical probes that exist in the literature have been shown to recognize and bind to their target lectins, however, they are accompanied with a number of limitations i.e. non-specific binding, photo bleaching, dimerization and oligomerization of labelled lectins. To date the development of carbohydrate-based chemical probes that efficiently detect lectins still requires investigation.

\subsubsection{Project Objectives}

The overall goal of this project is to develop efficient mannose-based chemical probes for use in the detection of mannose-binding lectins in various microorganisms. In particular, Actinobacteria will be examined. The Wakarchuk lab as well as others have shown there to be cell surface mannosylated proteins present in these microorganisms. ${ }^{63,64,65}$ These distinctive mannosylated proteins presented on their surfaces make them likely targets for lectins. To date, only a few lectincarbohydrate interactions have been elucidated for this group of bacteria. A few of its members such as Cellulomonas fimi (C.fimi) and Cellulomonas flavigena 
(C.flavigena) have, through genome sequencing, recently been suggested to containing a putative mannose-binding lectin, identified through sequence homology. ${ }^{66}$ Therefore, with the proposed mannose-based chemical probes this work explores whether mannose-binding lectins exist on the surfaces of these cells. This project is broken down into three aims:

1. Development and characterization of mannose-based chemical probes.

2. Investigation of the functionality of the mannose-based chemical probes on known mannose-binding lectins using flow cytometry and fluorescence microscopy techniques.

3. Using mannose-based chemical probes to discover the presence of mannose binding lectins on microorganisms.

For the development of mannose-based chemical probes, the recognition element of choice must consist of a mannose moiety (i.e. monosaccharides vs. disaccharides), for the specific interaction with target mannose-binding lectins. BODIPY fluorescent dyes will be the choice of tag owing to the properties previously discussed. For the linker component, PEG-based linkers will be used, as it should offset the hydrophobic properties of the BODIPY fluorescent tag (Figure 7). 

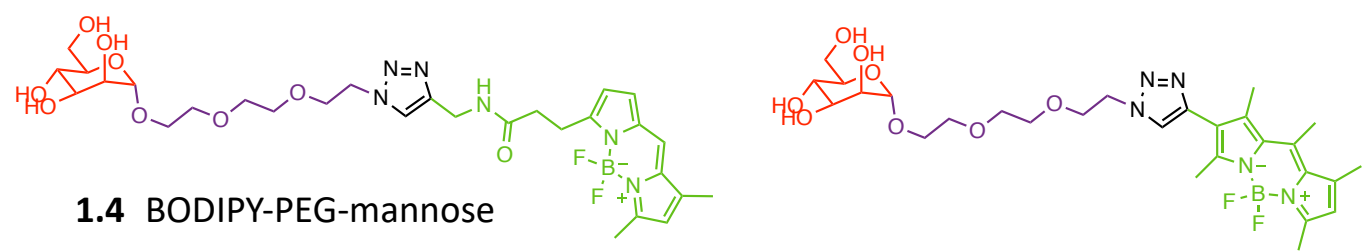

1.5 Planar-BODIPY-PEG-mannose

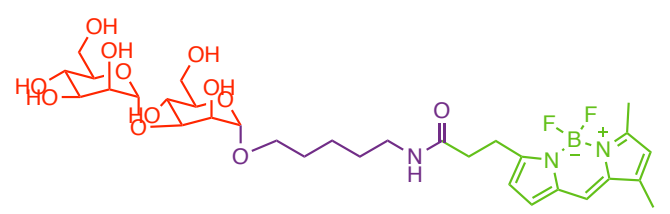

1.8 BODIPY-Mannobiose

Figure 7: Chemical structures of proposed BODIPY-mannose-based probes. Recognition element (red); linker (purple); and fluorescent tag (green).

It is hypothesized that the composition (tag, linker, and recognition element) of the developed mannose-based chemical probes will have an effect on its overall efficiency to specifically target and bind mannose-binding lectins. The fluorescent tag on the chemical probe will influence whether background labelling and nonspecific binding is observed. To investigate the functionality of these probes, tests will be done on Escherichia coli expressing FimH a known mannose binding surface lectin ${ }^{31,67}$ and examined through flow cytometry and fluorescence microscopy techniques.

Flow cytometry is a laser-based technology that uses light to count and sort cells based on the fluorescent characteristics of each cell in the fluid mixture. It will be used to study the binding selectivity of the mannose-based probes to mannosebinding lectin containing cells. Two sets of binding analyses will be conducted; i) a direct binding analysis, where the probe is directly incubated with cells and ii) an inhibition binding analysis, where a competitive ligand is introduced to inhibit the 
binding of the probe to cells. In theory, if the direct binding analysis is successful the data that is generated will show a peak shift to a higher fluorescence intensity for the population of cells bound to the probe in comparison to the unstained cells (negative control). Furthermore, if the inhibition binding analysis is successful the data that is generated will show a peak shift to lower fluorescence intensity since the binding of the probe to cells is inhibited, ultimately showing selectivity of the probe.

Fluorescence microscopy will be used to visually investigate the binding specificity of probes to cells. The key component of this method is the fluorescent dye as this is the molecule that is illuminated resulting in the excited fluorescent molecule to emit light that can be collected and visualized. Images produced will show the localization of the dye molecules, which provides information regarding specificity.

\subsubsection{Control Mannose-binding Lectin - FimH}

FimH are $30 \mathrm{kDa}$ mannose-binding lectins found on the organelle tip of Type 1 fimbriae of E.coli cells. ${ }^{31} \mathrm{FimH}$ is a well-studied lectin, produced as a 300 amino acid precursor and subsequently processed into a 279 amino acid mature form. ${ }^{67}$ These proteins are surface adhesion proteins of $E$. coli that are responsible for the specific binding of D-mannose containing host surfaces. ${ }^{32}$ Since, FimH lectins are known to specifically bind to mannose residues, they will be used as control lectins in this study to examine the functionality and efficiency of these mannose-based chemical 
probes, prior to using them to discover mannose-binding lectins on C.fimi and C.flavigena.

\subsection{Experimental}

\subsubsection{Materials}

Copper (II) sulfate pentahydrate, (+)-sodium L-ascorbate, tris(3hydroxypropyltriazolylmethyl)amine (THPTA), aminoguanidine hydrochloride, 7hydroxycoumarin-3-carboxylic acid N-succinimidyl ester (1.9) were all supplied by Sigma-Aldrich. C-18 silica gel columns were self-packed with silica powder also obtained from Sigma-Aldrich. Plastic-backed TLC silica gel 60 and glass-backed TLC plate (20 cm x $20 \mathrm{~cm} \times 1 \mathrm{~mm}$ thick) were purchased from EDM Millipore. All water used was obtained from the Milli-Q system also purchased from EDM Millipore. BODIPY FL alkyne (1.1) was purchased from Lumiprobe and planar BODIPY-alkyne (1.2) was supplied by the Koivisto Lab in the Department of Chemistry and Biology at Ryerson University. BODIPY-NHS (1.6) was supplied by the Withers Lab in the Department of Chemistry at University of British Columbia. $\alpha$-D-mannopyranosidePEG3-azide (1.3) was obtained from Sussex Research and D-mannose- $\alpha$-1,3mannosyl- $\alpha$-pentylamine (1.7) was supplied by GlycoNet Core Services. The prepacked Superdex peptide 10/300 GL column was purchased from GE Healthcare. The EZDOC System was purchased from BioRad. The vacuum concentrator and freeze drier equipment were purchased from Labconco. 


\subsubsection{General method for compound characterizations}

${ }^{1} \mathrm{H}$ NMR spectra were recorded at $400 \mathrm{MHz}$ on a Bruker Avance III 400 instrument at ambient temperature. Chemical shifts $(\delta)$ are reported in parts per million (ppm) from low to high field and references to a residual nondeuterated solvent $\left(\mathrm{H}_{2} \mathrm{O}\right)$ for ${ }^{1} \mathrm{H}$ nuclei. Standard abbreviations indicating multiplicity are used as follows: $\mathrm{s}=$ singlet; $\mathrm{d}=$ doublet; $\mathrm{m}=$ multiplet; $\mathrm{br}=$ broad. Mass spectra were obtained from an Advion mass spectroscopy instrument. Samples were introduced onto instrument via TLC/MS technique and run on an ESI probe. Methanol was used as the extracting solvent with flow rate of $0.15 \mathrm{~mL} / \mathrm{min}$. Full scan mass spectra were recorded in the positive-ion mode in a mass range of 100 to $2000 \mathrm{Da}$ applying the following parameters: detector gain 1250 , ESI voltage $3500 \mathrm{~V}$, capillary voltage $180 \mathrm{~V}$, source voltage offset $20 \mathrm{~V}$, source voltage span $30 \mathrm{~V}$, capillary temperature $250^{\circ} \mathrm{C}$, source gas temperature $200^{\circ} \mathrm{C}$.

\subsubsection{Purification technique using C-18 silica columns}

Excess reactant components were removed from the reaction mixture on C-18 silica columns. Prior to loading reaction mixture onto column, it was washed with $20 \mathrm{~mL}$ of methanol (MeOH) and $20 \mathrm{~mL}$ of water, successively. Subsequently, the reaction mixture was slowly loaded onto C-18 column and BODIPY-labelled probes were retained on the column. Un-bound components were eluted out the column during the wash step with $10 \mathrm{~mL}$ of water. While, the BODIPY-labelled probes were eluted with $\sim 4 \mathrm{~mL}$ of $\mathrm{MeOH}$. 


\subsubsection{Purification using preparative glass-backed TLC plates}

Total volume of reaction mixture was reduced to approximately $80 \mu \mathrm{L}$ using a vacuum concentrator. The concentrated reaction mixture was then layered onto a preparative glass-backed TLC plate $(20 \mathrm{~cm} \times 20 \mathrm{~cm} \times 1 \mathrm{~mm}$ thick $)$ and was developed using the solvent mix 4:2:1:0.1 (EtOAc:MeOH: $\mathrm{H}_{2} \mathrm{O}: \mathrm{HOAc}$ ). Developed TLC plate were allowed to completely dry in the dark at room temperature (RT) for 10-24 h. Once completely dry, band of interest were scraped off the (the mobility of probes are slightly different based on it being a monosaccharide or disaccharide) carefully with a Scoopula and transferred into a $50 \mathrm{~mL}$ conical tube. Probes were separated from the silica by washing the silica-bound probes with warm water $\left(40^{\circ} \mathrm{C}\right)$ until silica was completely clear and centrifuged at $1751 \mathrm{x} g$ at RT for $5 \mathrm{~min}$. Supernatant was gently decanted without including silica and subsequently purified following method outlined in section 2.3. The absorbance at $504 \mathrm{~nm}\left(\mathrm{ABS}_{504 \mathrm{~nm}}\right)$ was determined for the BODIPY-labelled product. $\mathrm{MeOH}$ was used as a blank and was used to dilute product. Obtained $\mathrm{ABS}_{504 \mathrm{~nm}}$ was then used to calculate the final concentration of the product (extinction coefficient $=80,000 \mathrm{~cm}^{-1} \mathrm{M}^{-1}$ ). Product was then completely dried down using a vacuum concentrator and stored at $-20^{\circ} \mathrm{C}$. 


\subsubsection{Synthesis and Purification of Mannose-based Probes}

\subsubsection{Synthesis of BODIPY-PEG-Mannose}

A 4 mM BODIPY-alkyne stock solution was prepared by dissolving BODIPY-alkyne (0.66 mg, $2 \mu \mathrm{mol}$ ) in $300 \mu \mathrm{L}$ of DMF and $200 \mu \mathrm{L}$ of water. A $20 \mathrm{mM} \alpha-\mathrm{D}-$ mannopyranoside-PEG3-azide (1.3) stock solution was prepared by dissolving $\alpha$-Dmannopyranoside-PEG3-azide (1.3) $(2.67 \mathrm{mg}, 8 \mu \mathrm{mol})$ in $400 \mu \mathrm{L}$ of water. A $2 \mathrm{mM}$ $\mathrm{CuSO}_{4}$ stock solution was prepared by dissolving $\mathrm{CuSO}_{4}(0.50 \mathrm{mg}, 2 \mu \mathrm{mol}) \mathrm{in} 1 \mathrm{ml}$ of water. A $50 \mathrm{mM}$ ligand THPTA stock solution was prepared by dissolving ligand THPTA (35.7 mg, $82.2 \mu \mathrm{mol}$ ) in $2 \mathrm{ml}$ of water. A $100 \mathrm{mM}$ aminoguanidine stock solution was prepared by dissolving aminoguanidine (13.1 $\mathrm{mg}, 0.12 \mathrm{mmol}$ ) in $1 \mathrm{ml}$ of water. And $100 \mathrm{mM}$ sodium ascorbate stock solution was prepared by dissolving sodium ascorbate ( $24.9 \mathrm{mg}, 0.13 \mathrm{mmol})$ in $1 \mathrm{ml}$ of water. The BODIPY-PEG-mannose probe was synthesized via a CuAAc reaction under the following assay conditions: 2 mM BODIPY alkyne, potassium phosphate buffer $\mathrm{pH} 7$ to make up the total volume, $4 \mathrm{mM}$ Man-PEG-azide, $0.25 \mathrm{mM} \mathrm{CuSO}_{4}, 1.25 \mathrm{mM}$ ligand, $5 \mathrm{mM}$ aminoguanidine and 5 mM sodium ascorbate. Once all reagents were combined, the reaction was incubated at $37^{\circ} \mathrm{C}$ in the dark. Progress of the reaction was monitored on a plastic-backed TLC plate (silica 60) until full conversion of product formation was observed. $0.5 \mu \mathrm{L}$ of the reaction was spotted on a TLC plate and was developed using a 4:2:1:0.1 mix of EtOAc:MeOH: $\mathrm{H}_{2} \mathrm{O}: \mathrm{HOAc}$ solvent. The developed TLC plate was placed on a blue light tray $\left(\lambda_{\mathrm{ex}}=460 \mathrm{~nm}\right)$ and was then inserted into the EZDOC imaging system for band quantitation. The resulting reaction mixture was then purified using the method 
outlined in section 2.4. From a $1 \mathrm{~mL}$ reaction $\sim 5 \mathrm{mg}$ of product was obtained. ${ }^{1} \mathrm{H}$ NMR (400 MHz, $\left.\mathrm{D}_{2} \mathrm{O}\right): \delta=7.94(\mathrm{~s}, 1 \mathrm{H}), 7.56(\mathrm{~s}, 1 \mathrm{H}), 7.42(\mathrm{~s}, 1 \mathrm{H}), 7.01(\mathrm{~s}, 1 \mathrm{H}), 6.31(\mathrm{~s}$, 1H), $4.90(\mathrm{~s}, 1 \mathrm{H}), 4.83(\mathrm{~s}, 2 \mathrm{H}), 4.53-4.41(\mathrm{~m}, 5 \mathrm{H}), 3.98-3.51(\mathrm{~m}, 17 \mathrm{H}), 3.20(\mathrm{br}$, 2H), $2.72(\mathrm{br}, 2 \mathrm{H}), 2.51(\mathrm{~s}, 3 \mathrm{H}), 2.27(\mathrm{~s}, 3 \mathrm{H})$.

TLC-MS (ESI): $\mathrm{C}_{29} \mathrm{H}_{41} \mathrm{BF}_{2} \mathrm{~N}_{6} \mathrm{O}_{9}[\mathrm{M}+\mathrm{Na}]^{+}$, calculated $m / z=689.48$, found $m / z$ 689.4.

\subsubsection{Synthesis of BODIPY-Mannobiose}

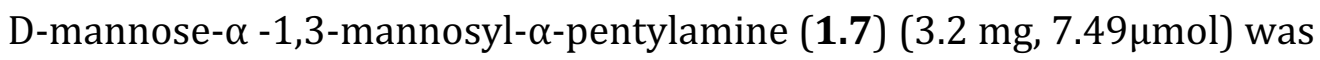

resuspended in $150 \mu \mathrm{L}$ of sodium borate ( $25 \mathrm{mM}, \mathrm{pH}$ 8.5). In a separate microcentrifuge tube BODIPY-NHS (1.6) (3 mg, 7.7 $\mu \mathrm{mol}$ ) was dissolved in $154 \mu \mathrm{L}$ of dimethylformamide (DMF). The two components were mixed together in a single microcentrifuge tube and incubated at room temperature with constant rotating for 3-24h. The progress of the reaction was monitored on a plastic-backed TLC plate until full conversion of product formation was observed. $0.5 \mu \mathrm{L}$ of the reaction was spotted on a TLC plate and was developed using a 4:3:2:0.1 mix of EtOAc:MeOH: $\mathrm{H}_{2} \mathrm{O}: \mathrm{HOAc}$ solvent. Quantification of the developed TLC plate was done on a blue light tray $\left(\lambda_{\mathrm{ex}}=460 \mathrm{~nm}\right)$, which was inserted into the EZDOC imaging system. The reaction mixture was then purified using the method outlined in section 2.4. From a $1 \mathrm{~mL}$ reaction $\sim 4.3 \mathrm{mg}$ of product was obtained. ${ }^{1} \mathrm{H}$ NMR $(400 \mathrm{MHz}$, $\left.\mathrm{D}_{2} 0\right): \delta=7.32(\mathrm{~s}, 1 \mathrm{H}), 6.97(\mathrm{~s}, 1 \mathrm{H}), 6.32(\mathrm{~s}, 1 \mathrm{H}), 6.18(\mathrm{~s}, 1 \mathrm{H}), 5.12(\mathrm{br}, 2 \mathrm{H}), 4.08-$ $4.05(\mathrm{~m}, 2 \mathrm{H}), 3.88-3.75(\mathrm{~m}, 9 \mathrm{H}), 3.70-3.59(\mathrm{~m}, 3 \mathrm{H}), 3.37(\mathrm{br}, 2 \mathrm{H}), 3.16-3.12(\mathrm{~m}$, 4H), $2.45(\mathrm{~s}, 1 \mathrm{H}), 2.16(\mathrm{~s}, 1 \mathrm{H}), 1.48-1.39(\mathrm{~m}, 5 \mathrm{H}), 1.21-1.10(\mathrm{~m}, 6 \mathrm{H})$. TLC-MS (ESI): $\mathrm{C}_{31} \mathrm{H}_{46} \mathrm{BF}_{2} \mathrm{~N}_{3} \mathrm{O}_{12}[\mathrm{M}+\mathrm{Na}]^{+}$, calculated $m / z$ 724.30, found $m / z$ 724.5. 


\subsubsection{Synthesis of Coumarin-Mannobiose}

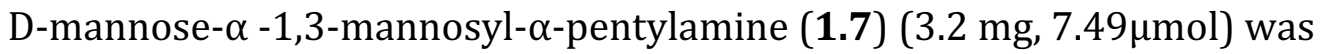
resuspended in $150 \mu \mathrm{L}$ of sodium borate ( $25 \mathrm{mM}$, pH 8.5). In a separate microcentrifuge tube, 7-hydroxycoumarin-3-carboxylic acid N-succinimidyl ester (1.9) (2.3 mg, $7.6 \mu \mathrm{mol})$ was dissolved in $152 \mu \mathrm{L}$ of dimethylformamide (DMF). The two components were mixed together in a single microcentrifuge tube and incubated at room temperature with constant rotating for 10-24h. The progress of the reaction was monitored on a plastic-backed TLC plate until full conversion of product formation was observed. $0.5 \mu \mathrm{L}$ of the reaction was spotted on a TLC plate and was developed using a 4:3:2:0.1 mix of EtOAc:MeOH: $\mathrm{H}_{2} \mathrm{O}: \mathrm{HOAc}$ solvent. Quantification of the developed TLC plate was done on a blue light tray $\left(\lambda_{\mathrm{ex}}=\right.$ $460 \mathrm{~nm}$ ), which was inserted into the EZDOC imaging system. Purification was done on a superdex peptide $10 / 300 \mathrm{GL}$ column attached to a ÄKTA pure chromatography system (GE Healthcare). Resulting reaction mixture was diluted with $10 \mathrm{mM}$ ammonium acetate $+20 \%$ acetonitrile $(\mathrm{ACN}) \mathrm{pH} 7.5$ buffer in a 1:1(v/v). Diluted reaction mixture was loaded onto column at $1 \mathrm{~mL} / \mathrm{min}$ flow rate. The product was eluted at $1 \mathrm{~mL} / \mathrm{min}$ with a isocratic flow of $10 \mathrm{mM}$ ammonium acetate $+20 \%$ ACN pH 7.5 buffer and $2 \mathrm{~mL}$ fractions were collected. The eluted product was then analyzed on a plastic-backed TLC plate to confirm the elimination of impurities. The purified coumarin-mannobiose fractions were then pooled and completely dried down using a freeze dryer and final product was stored at $-20^{\circ} \mathrm{C}$. From a $1 \mathrm{~mL}$ reaction $\sim 4.0 \mathrm{mg}$ of product was obtained. ${ }^{1} \mathrm{H}$ NMR $\left(400 \mathrm{MHz}, \mathrm{D}_{2} \mathrm{O}\right): \delta=8.55(\mathrm{~s}$, 1H), $7.59(\mathrm{~s}, 1 \mathrm{H}), 6.88(\mathrm{~s}, 1 \mathrm{H}), 6.73(\mathrm{~s}, 1 \mathrm{H}), 5.11(\mathrm{~s}, 1 \mathrm{H}), 4.85(\mathrm{~m}, 2 \mathrm{H}), 4.08-4.05(\mathrm{~m}$, 
2H), $3.90-3.83(\mathrm{~m}, 2 \mathrm{H}) 3.78-3.55(\mathrm{~m}, 9 \mathrm{H}), 3.38(\mathrm{t}, 2 \mathrm{H}), 2(\mathrm{~s}, 2 \mathrm{H}), 1.67-1.64(\mathrm{~m}$,

5H), $1.48-146(\mathrm{~m}, 6 \mathrm{H})$. TLC-MS (ESI): $\mathrm{C}_{27} \mathrm{H}_{37} \mathrm{NO}_{15}[\mathrm{M}+\mathrm{Na}]^{+}$, calculated $m / z$ 638.21, found $m / z 638.3$.

\subsubsection{Synthesis of BODIPY-PEG-amine}

A 20 mM azido-PEG3-amine stock solution was prepared by dissolving azido-PEG3amine (1.3 mg, $6 \mu \mathrm{mol})$ in $300 \mu \mathrm{L}$ of $25 \mathrm{mM}$ sodium borate $\mathrm{pH}$ 8.5. The CuAAC reaction protocol outlined in section 2.5.1 was used. The progress of the reaction was monitored on a plastic-backed TLC plate until full conversion of product formation was observed. $0.5 \mu \mathrm{L}$ of the reaction was spotted on a TLC plate and was developed using a 4:2:1:0.1 mix of EtOAc:MeOH: $\mathrm{H}_{2} \mathrm{O}: \mathrm{HOAc}$ solvent. The developed TLC plate was then analyzed on the EZDOC system using a blue light tray for band quantification. The reaction mixture was then purified using the method outlined in section 2.4. From a $1 \mathrm{~mL}$ reaction $\sim 2 \mathrm{mg}$ of product was obtained. NMR and MS analysis will be done prior to final submission of thesis. 


\subsubsection{Growth of Bacteria}

\subsubsection{Growth conditions for C. fimi and C. flavigina}

C. fimi wild-type strain (ATCC 484) was streaked onto a plate containing low sodium lennox broth agar media. Plate was incubated at $30^{\circ} \mathrm{C}$ for $48 \mathrm{~h}$. A colony from the plate was used to inoculate $25 \mathrm{~mL}$ of $2 \mathrm{x}$ YT media in a $125 \mathrm{~mL}$ baffled flask. The flask was then incubated at $30^{\circ} \mathrm{C}$ with shaking at $180 \mathrm{rpm}$ for $30 \mathrm{~h}$ or until cells have reached an optical density at $\lambda 600 \mathrm{~nm}\left(\mathrm{OD}_{600}\right)$ of 1 . Cells were then transferred to a microcentrifuge tube and centrifuged at 13,000 x-g for $10 \mathrm{~min}$. The supernatant was discarded and the cells were washed twice with 1X PBS. Washed pellet was resuspended with $1 \mathrm{X}$ PBS and cell mixture was diluted to a target $\mathrm{OD}_{600} \mathrm{C}$. flavigina wild-type (ATCC 482) was also grown using the same conditions as C. fimi.

\subsubsection{Growth conditions for FimH E.coli and FimH mutant E.coli}

FimH E.coli wild-type strain (NRG857c) was scrapped off a frozen glycerol stock (supplied by the McPhee Lab at Ryerson in the Department of Chemistry and Biology at Ryerson University) with a pipette tip and used to inoculate $3 \mathrm{~mL}$ of low sodium LB and it was incubated at $37^{\circ} \mathrm{C}$ with shaking at $200 \mathrm{rpm}$ for $24 \mathrm{hr}$. The next day a subculture was made with 1/50 dilution from the overnight into a $3 \mathrm{~mL}$ low sodium $\mathrm{LB}$ and incubated at $37^{\circ} \mathrm{C}$ with shaking at $200 \mathrm{rpm}$ for $\sim 2 \mathrm{~h}$ or until cells have reached an $\mathrm{OD}_{600}=1$. Cells were then transferred to a microcentrifuge tube and centrifuged at 13,000 x-g for $10 \mathrm{~min}$. The supernatant was discarded and the 
cells were washed twice with 1X PBS. Washed pellet was resuspended with 1X PBS. FimH mutant E.coli strain (NRG857c $\Delta$ FimH) (supplied by the McPhee Lab at Ryerson in the Department of Chemistry and Biology at Ryerson University) was also grown using the same conditions as FimH E.coli.

\subsubsection{Flow Cytometry Analysis}

All flow cytometry analyses were done on the BD Accuri C6 plus flow cytometer instrument (BD Biosciences). Pre-set flow rate and core size used were $14 \mu \mathrm{L} / \mathrm{min}$ and $10-\mu \mathrm{m}$ core, respectively. Laser excitation used was $488 \mathrm{~nm}$ and emission used was $530 \pm 15 \mathrm{~nm}$. Total events analyzed per sample was 50,000 - 100,000. All flow cytometry analyses were conducted in triplicates.

\subsubsection{Direct \& Inhibition Binding Preparation with PFA fixation}

After bacterial growth to target $\mathrm{OD}_{600}$, cells were washed with $1 \mathrm{x}$ PBS, centrifuged at $13,000 \mathrm{x}$-g for $10 \mathrm{~min}$ to remove media. For the direct binding analysis, $200 \mu \mathrm{L}$ cells at target $\mathrm{OD}_{600}$ were first incubated with probe $(25 \mu \mathrm{M}$ or $100 \mu \mathrm{M})$ for $1 \mathrm{~h}$ in a microcentrifuge tube in the dark at room temperature. Mixture was then washed $3 \mathrm{X}$ with $1 \mathrm{x}$ PBS by centrifugation to remove unbound probe and the supernatant was discarded. Bacterial cell pellet was fixed with PFA ( $2 \%$ or $4 \%$ ) for $20 \mathrm{~min}$ and then washed $3 \mathrm{X}$ with $1 \mathrm{X}$ PBS by centrifugation. Supernatant was discarded and cells were resuspended in $0.4 \mathrm{~mL} 1 \mathrm{x}$ PBS for flow cytometry analysis. 
For inhibition binding analysis, the protocol described for direct binding assay was used, however an additional step was introduced prior to PFA fixation. In this step, a competitive ligand $(1 \mathrm{mM})$ was incubated with the cells for $1 \mathrm{~h}$ in a microcentrifuge tube in the dark at room temperature.

\subsubsection{Direct \& Inhibition Binding Preparation with $\mathrm{NaN}_{3}$ treatment}

After bacterial growth to target $\mathrm{OD}_{600}$, cells were then washed with 1x PBS and centrifuged at 13,000 x-g for $10 \mathrm{~min}$ to remove media. For the direct binding analysis, bacterial cells were treated with $1 \mathrm{mM} \mathrm{NaN}_{3}$ for $\sim 15-20 \mathrm{~min}$. Cells were then washed 3 times with 1x PBS by centrifugation and supernatant was discarded. Cells were re-suspended in $1 \mathrm{x}$ PBS and the final $\mathrm{OD}_{600}$ was measured. Probe $(25 \mu \mathrm{M}$ or $0.1 \mathrm{mM}$ ) was then added to the $200 \mu \mathrm{L}$ cells at target $\mathrm{OD}_{600}$ and incubated for $1 \mathrm{~h}$ in a microcentrifuge tube in the dark at room temperature. Cells were then washed $3 \mathrm{X}$ with 1X PBS by centrifugation to remove unbound probe. Supernatant was discarded and cells were resuspended in $0.4 \mathrm{~mL} 1 \mathrm{x}$ PBS for flow cytometry analysis.

For Inhibition binding analysis, the protocol described for direct binding assay was used, however and additional step was incorporated after the step with the excess probe washed off. In this step, a competitive ligand (1mM) was incubated with the cells for $1 \mathrm{~h}$ in a microcentrifuge tube in the dark at room temperature. 


\subsubsection{Fluorescence Microscopy}

All fluorescence microscopy images were acquired from a deconvolution fluorescence microscope (Olympus). Three preparation methods were used:

Method 1: FimH E.coli and FimH mutant E.coli cells $\left(\mathrm{OD}_{600}=1\right)$ were incubated with $6 \mu \mathrm{M}$ or $25 \mu \mathrm{M}$ probe in a microcentrifuge tube for $1 \mathrm{~h}$ in the dark at room temperature. $20 \mu \mathrm{L}$ of mixture was transferred onto coverslips in a 6-well plate. The sample was then allowed to air dry onto the coverslip for $\sim 3 \mathrm{~h}$. Excess probe was washed off $3 \mathrm{X}$ with $1 \mathrm{x}$ PBS with $5 \mathrm{~min}$ of gentle shaking. Cells were then fixed with 2\% PFA for $20 \mathrm{~min}$ and then washed $3 \mathrm{X}$ with $1 \mathrm{x}$ PBS. Coverslips were then mounted on the glass slides with a drop of DAKO, which was then allowed to dry $10-24 \mathrm{~h}$ in the dark.

Method 2: C. fimi and C. flavigina cells $\left(\mathrm{OD}_{600} \approx 0.23\right)$ were incubated with 25 $\mu \mathrm{M}$ probe in microcentrifuge tube for $1 \mathrm{~h}$ in the dark at room temperature. Excess probe was washed off $3 \mathrm{X}$ with $1 \mathrm{x}$ PBS. Cells were fixed with 2\% PFA for 20min and washed $3 X$ with 1x PBS. Fixed cells were co-stained with DAPI for $15 \mathrm{~min}$ and washed excess off $3 \mathrm{X}$ with $1 \mathrm{x}$ PBS. Stained bacterial cells were then prepared for imaging using an agarose pad method outlined in a previously published procedure ${ }^{33}$ However, the coverslip/agarose pad set-up was optimized as follows, from bottom to top: glass slide, agarose pad, $2 \mu \mathrm{L}$ bacterial culture, and coverslip.

Method 3: C. fimi and C.flavigena cells $\left(\mathrm{OD}_{600} \approx 0.23\right)$ were first treated with 1 $\mathrm{mM} \mathrm{NaN}_{3}$ for 15-20 min. Cells were then washed $3 \mathrm{X}$ with $1 \mathrm{x}$ PBS by centrifugation and supernatant was discarded. Cells were then incubated with $0.1 \mathrm{mM}$ probe in a microcentrifuge tube for $1 \mathrm{~h}$ in the dark at room temperature. Excess probe was 
washed off $3 \mathrm{X}$ with $1 \mathrm{x}$ PBS. Cells were then co-stained with DAPI for 15min and washed excess off $3 \mathrm{X}$ with $1 \mathrm{x}$ PBS. Stained bacterial cells were then prepared for imaging using an agarose pad method outlined in a previously published procedure. ${ }^{33}$ However, the coverslip/agarose pad set-up was optimized as follows, from bottom to top: glass slide, agarose pad, $2 \mu \mathrm{L}$ bacterial culture, and coverslip. 


\subsection{Results}

\subsubsection{Development of Mannose-based Probes}

\subsubsection{Synthesis of BODIPY-PEG-Mannose Probes}

Both mannose-based chemical probes were synthesized using the CuAAC reaction outlined in Figure 5, which labelled the $\alpha$-D-mannopyranoside-PEG3-azide (1.3) with either the lumiprobe BODIPY-alkyne (1.1) or the planar BODIPY-alkyne (1.2) (Figure 8A). From the TLC results obtained, about 95\% of the BODIPY-alkyne (1.1) was successfully 'clicked' onto the $\alpha$-D-mannopyranoside-PEG3-azide within $2 \mathrm{~h}$ of incubation forming BODIPY-PEG-mannose (1.4) (Figure 8B). Whereas, only about $\sim 44.8 \%$ of the BODIPY-alkyne (1.2) was 'clicked' onto the $\alpha$-D-mannopyranosidePEG3-azide within $10 \mathrm{~h}$ of incubation generating a directly conjugated BODIPY-PEGmannose (1.5) (Figure 8C). No further product formation with the BODIPY (1.2) tag was observed even after 72 hours of incubation.

A)

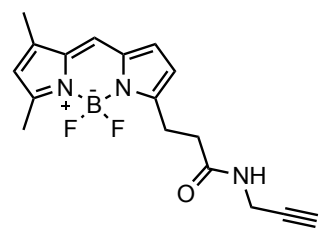

1.1 BODIPY-alkyne

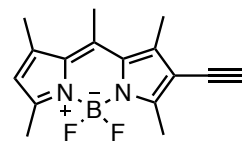

1.2 Planar BODIPY-alkyne

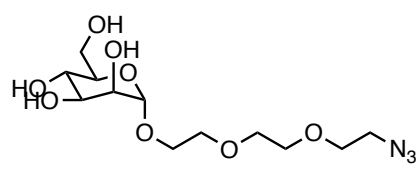

1.3

$\alpha$-D-mannopyranoside-PEG3-azide 
B)

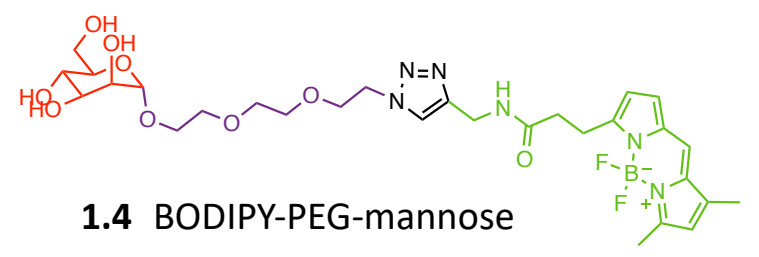

1.4 BODIPY-PEG-mannose

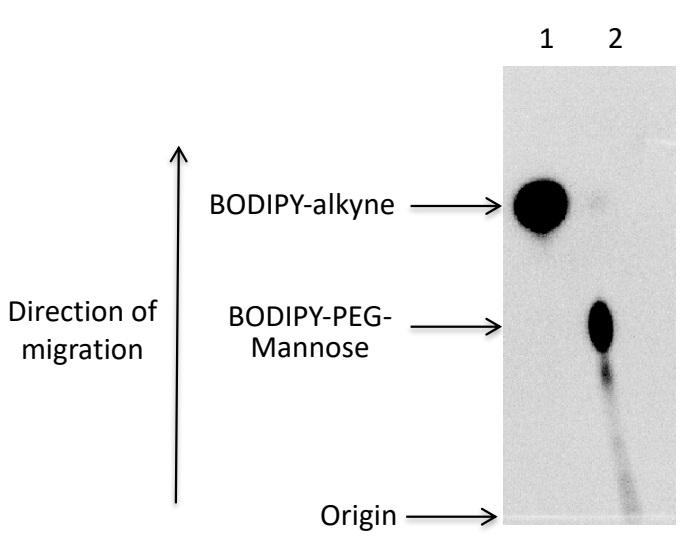

C)

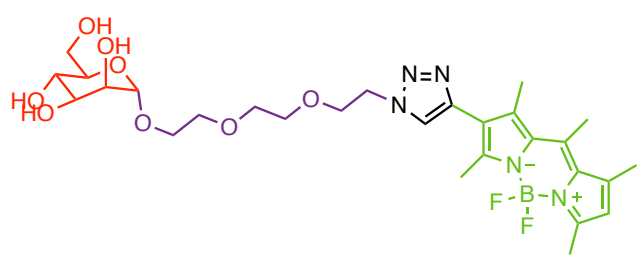

1.5 Planar-BODIPY-PEG-mannose

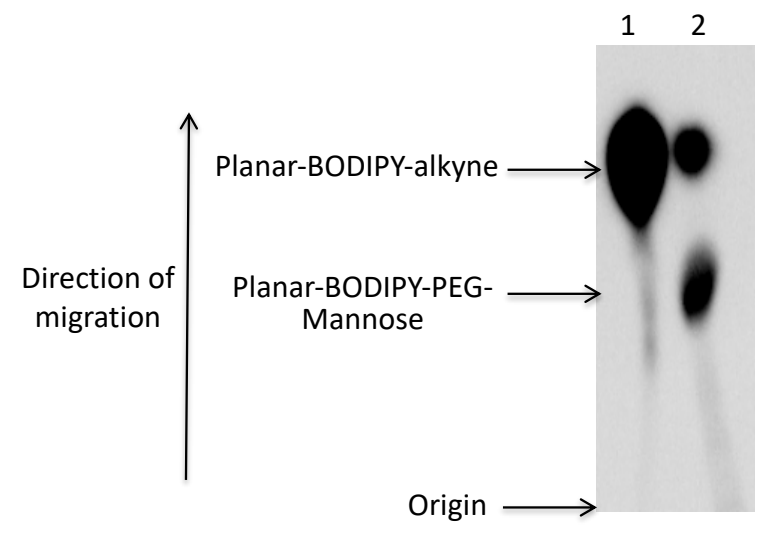

Figure 8: Synthesis of BODIPY-PEG-Mannose Probes. A) Chemical structures of the starting materials used in the CuAAc reaction. B) TLC analysis of BODIPY-PEGmannose (1.4) synthesis. Lane 1: starting material, BODIPY-alkyne; Lanes 2: CuAAC reaction mixture, time point: $2 h$. C) TLC analysis of Planar-BODIPY-PEG-mannose (1.5) synthesis. Lane 1: starting material, planar BODIPY-alkyne; Lanes 2: CuAAc reaction mixture, time point: $10 \mathrm{~h}$.

\subsubsection{Synthesis of BODIPY-Mannobiose probe}

The BODIPY-mannobiose (1.8) probe was synthesized using the succinimidyl ester reaction. In this reaction, the amine-reactive BODIPY dye was reacted with the Dmannose- $\alpha$-1,3-mannosyl- $\alpha$-pentylamine (1.7) under alkaline conditions 
generating a stable amide linked BODIPY labelled mannobiose product illustrated in Figure 9A. From the TLC results obtained, about $85 \%$ relative conversion was achieved within $3 \mathrm{~h}$ of incubation and labelled product was then successfully purified via preparative TLC (Figure 9B).

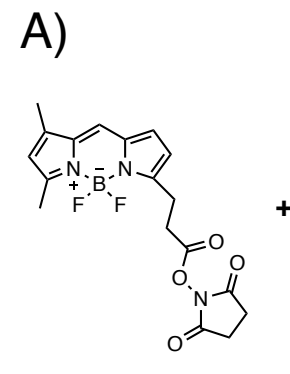

1.6 BODIPY-NHS

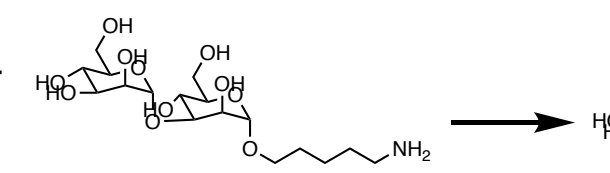

1.7

D-Mannose- $\alpha-1,3-$ Mannosyl- $\alpha$-pentylamine

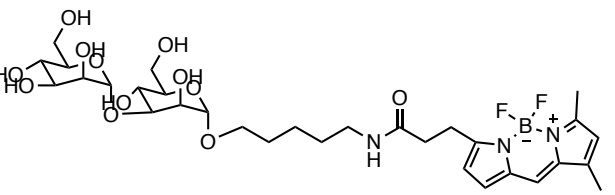

1.8 BODIPY-Mannobiose

B)

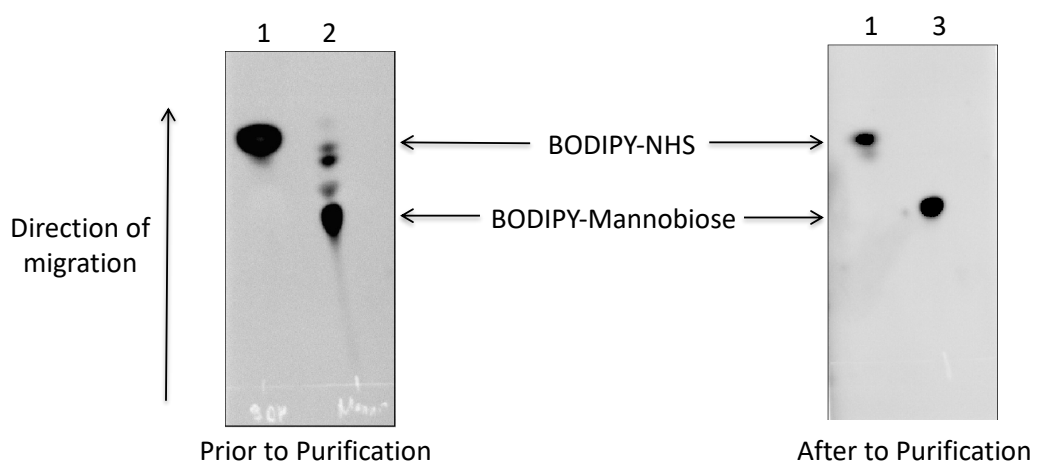

Figure 9: Synthesis of BODIPY-Mannobiose. A) Succinimidyl ester reaction scheme of BODIPY-mannobiose and B) TLC analysis of BODIPY-mannobiose synthesis. Lane 1: starting material, BODIPY-NHS; Lanes 2: reaction mixture, time point: 3 h; Lane 3: purified BODIPY-mannobiose fraction.

\subsubsection{Synthesis of Coumarin-Mannobiose}

The coumarin-mannobiose (1.10) probe was synthesized using the succinimidyl ester reaction as well, illustrated in Figure 10. From the TLC results obtained, about $75 \%$ relative conversion was achieved within $22 \mathrm{~h}$ of incubation as seen in Lane 2 of 
Figure 11B. The mixture was then successfully purified via size-exclusion chromatography, where four different fractions (Fractions a-b) were observed to elute from the column (Figure 11A). The coumarin-mannobiose probe successfully eluted first due to its larger size (fraction a), which was confirmed by TLC as shown in Lane 3 on Figure 11B.

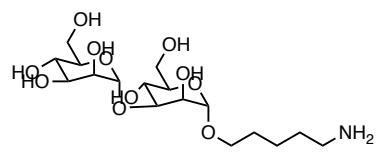

1.7

D-Mannose-a-1,3Mannosyl-a-pentylamine

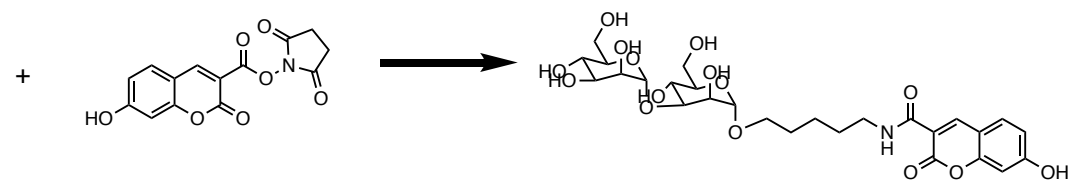

7-hydroxycoumarin-3carboxylic-NHS
1.10 Coumarin-mannobiose

Figure 10: Succinimidyl ester reaction scheme of coumarin-mannobiose 
A)

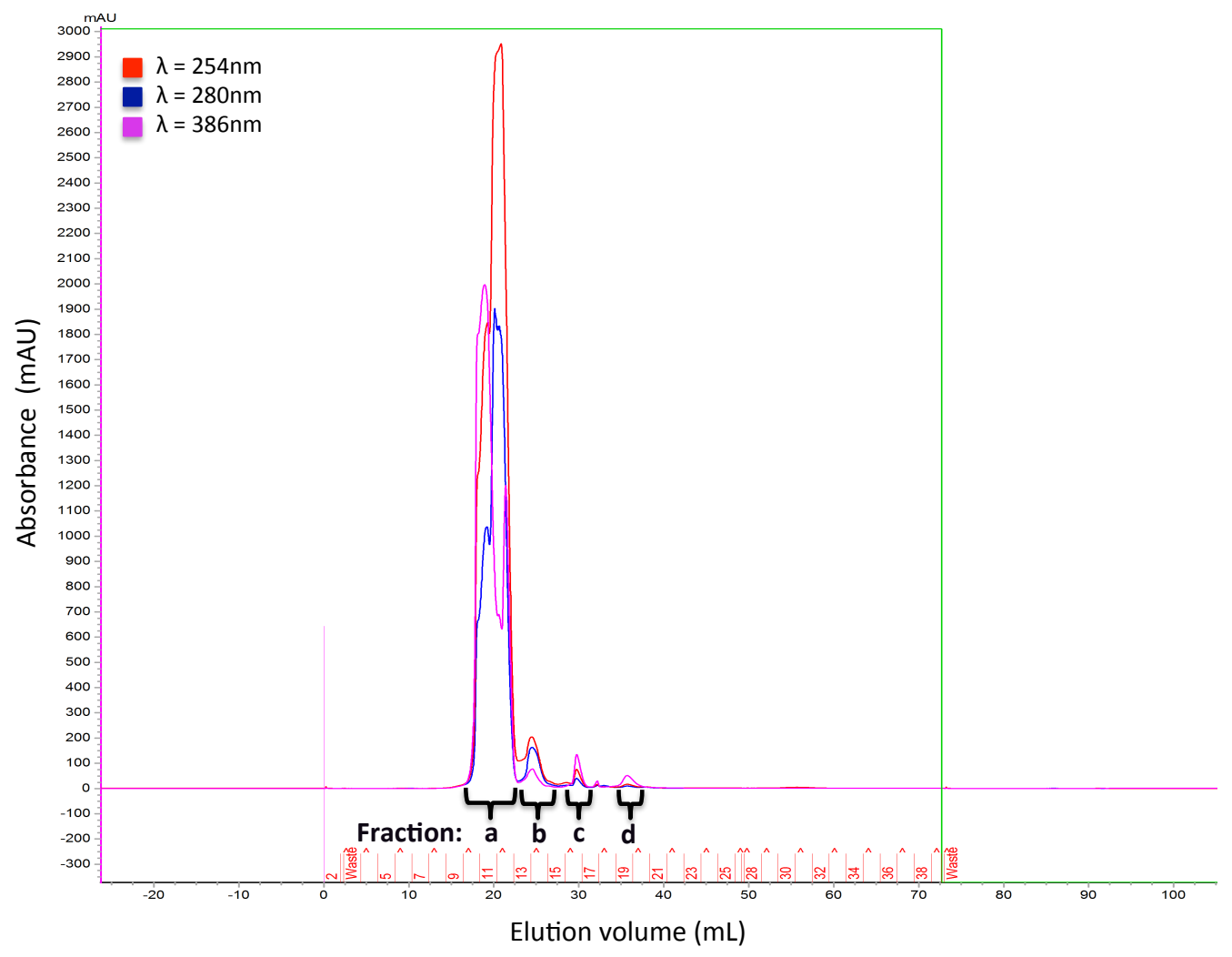

B)

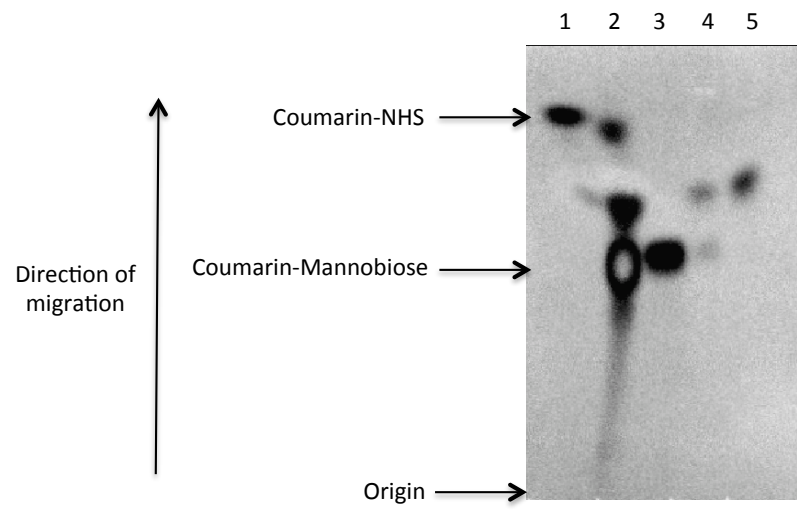

Figure 11: Synthesis of Coumarin-Mannobiose. A) Purification of coumarinmannobiose by size-exclusion chromatography and B) TLC analysis of coumarinmannobiose reaction mixture and fractions from purification. Lane 1: starting material, Coumarin-NHS; Lanes 2: reaction mixture, time point: $22 \mathrm{~h}$; Lanes 3-5: fractions from purification. Lane 3: fraction a (purified coumarin-mannobiose); Lane 4: fraction b; and Lane 5: fraction $c$. 


\subsubsection{Flow Cytometry Analysis}

\subsubsection{Direct binding and inhibition binding of mannose probes on FimH E.coli}

In order to investigate the selectivity of BODIPY-PEG-mannose probe for FimH lectins, a direct binding analysis was conducted. Alongside the control FimH E.coli cells (NRG857c), the direct binding analysis was also conducted on FimH mutant E.coli cells (NRG857c $\Delta$ FimH) under identical conditions. In the analysis, a BODIPYPEG-amine compound, missing the mannose recognition element, was also incorporated into the study to investigate whether there was non-specific binding occurring with the BODIPY-PEG component of the probe. From the histogram results shown in Figure 12A, its evident that the BODIPY-PEG-mannose probe is binding to the FimH E.coli cells due to the $\sim 8.3$ fold increase in fluorescence intensity compared to the unstained cells. In addition, a slight fluorescence intensity shift was also observed for the cells incubated with BODIPY-PEG-amine, suggesting that the BODIPY-PEG region of the probe was a bit sticky. The histogram obtained for the FimH mutant E.coli cells in Figure 12B, shows that the selectivity of the BODIPY-PEG-mannose probe is specifically for FimH lectins as similar shift were not observed with the FimH mutant E.coli cells. However, this histogram confirmed that the BODIPY-PEG component of the probe was in fact 'sticky' with both the BODIPYPEG-amine and BODIPY-PEG-mannose probe having the same fluorescence intensity shift in the FimH mutant E.coli cells. This 'stickiness' observed might be the hydrophobic or hydrogen bond interactions that might be occurring between the BODIPY-PEG component and cell surface components. 
A)

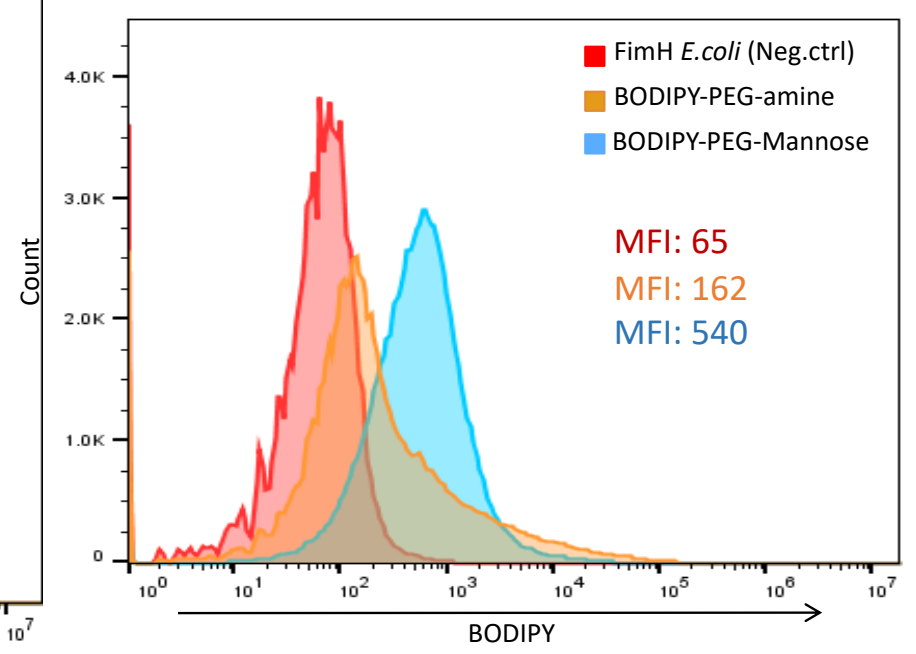

B)

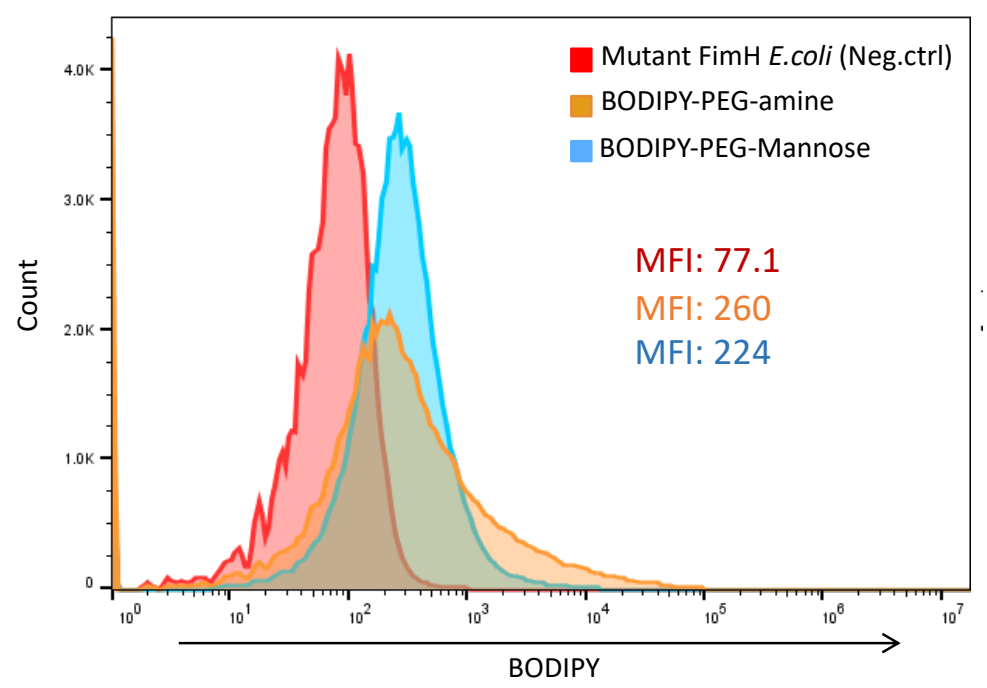

Figure 12: Investigating the binding specificity of BODIPY-PEG-Mannose probe for FimH lectins by flow cytometry. Direct binding analyses of probes binding to A) FimH E.coli cells $\left(O D_{600}=1\right)$ and B) Fim H mutant E.coli cells $\left(O D_{600}=1\right)$. Negative control, probe absent (red); cells incubated with 25 MM BODIPY-PEG-Mannose amine (orange); and cells incubated with $25 \mu$ M BODIPY-PEG-Mannose probe (blue). MFI: median fluorescence intensity. 
To further investigate the selectivity of the BODIPY-PEG-mannose probe for FimH lectins, an inhibition binding analysis was carried out, which introduced a competitive ligand. This analysis was done to investigate whether the binding of the probe to the FimH E.coli cells would be inhibited resulting in a decrease in fluorescence intensity. The competitive ligand selected for the analysis was the 4nitrophenyl $\alpha$-D-mannopyranoside, known for specifically binding to FimH lectins. From the histogram obtained, inhibition activity was observed with the competitive ligand. A decrease in fluorescence intensity was seen in comparison to the shift with just the BODIPY-PEG-mannose probe (Figure 13). Similar analysis was also conducted on FimH mutant E.coli cells, resulting in no inhibition activity, instead relatively similar fluorescence intensity shifts were observed for the BODIPY-PEGamine, BODIPY-PEG-mannose probe and competitive ligand due to the "stickiness" of the probes (Figure 14). 


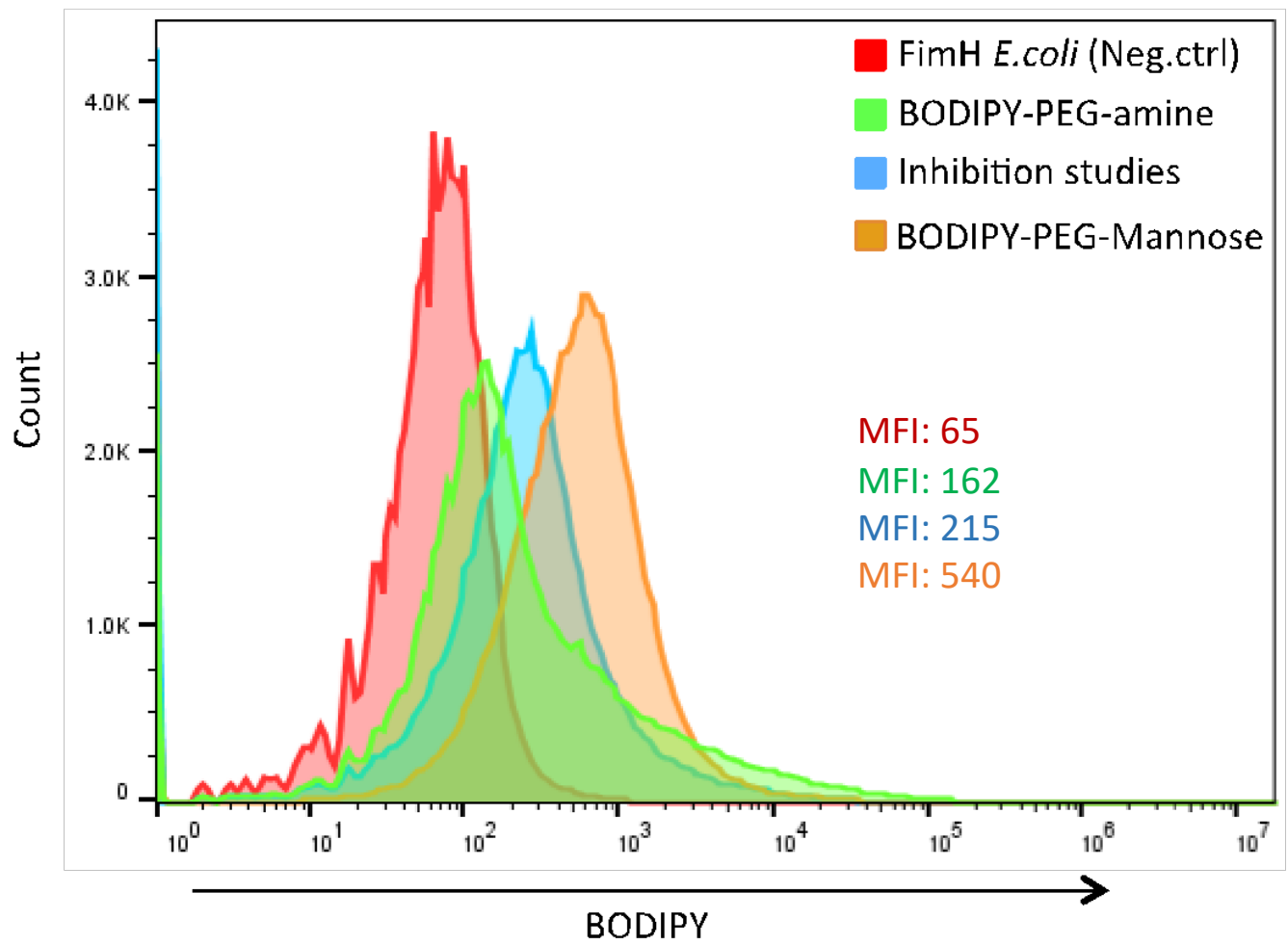

Figure 13: Inhibition binding studies to investigate the selectivity of BODIPY-PEGMannose probe binding to FimH E.coli cells by flow cytometry. Negative control, probe absent (red). Non-specific binding of $25 \mu \mathrm{M}$ control BODIPY-PEG-amine probe to FimH E.coli cells (green). Selective binding of $25 \mu$ M BODIPY-PEG-mannose probe to FimH E.coli cells (orange). Inhibition of this selective binding by the competitive ligand: 4nitrophenyl $\alpha$-D-mannopyranoside (blue). Analyses were all done with FimH E.coli cells at $O D_{600}=1$. For each sample the analysis of 100,000 events were done. MFI: median fluorescence intensity. 


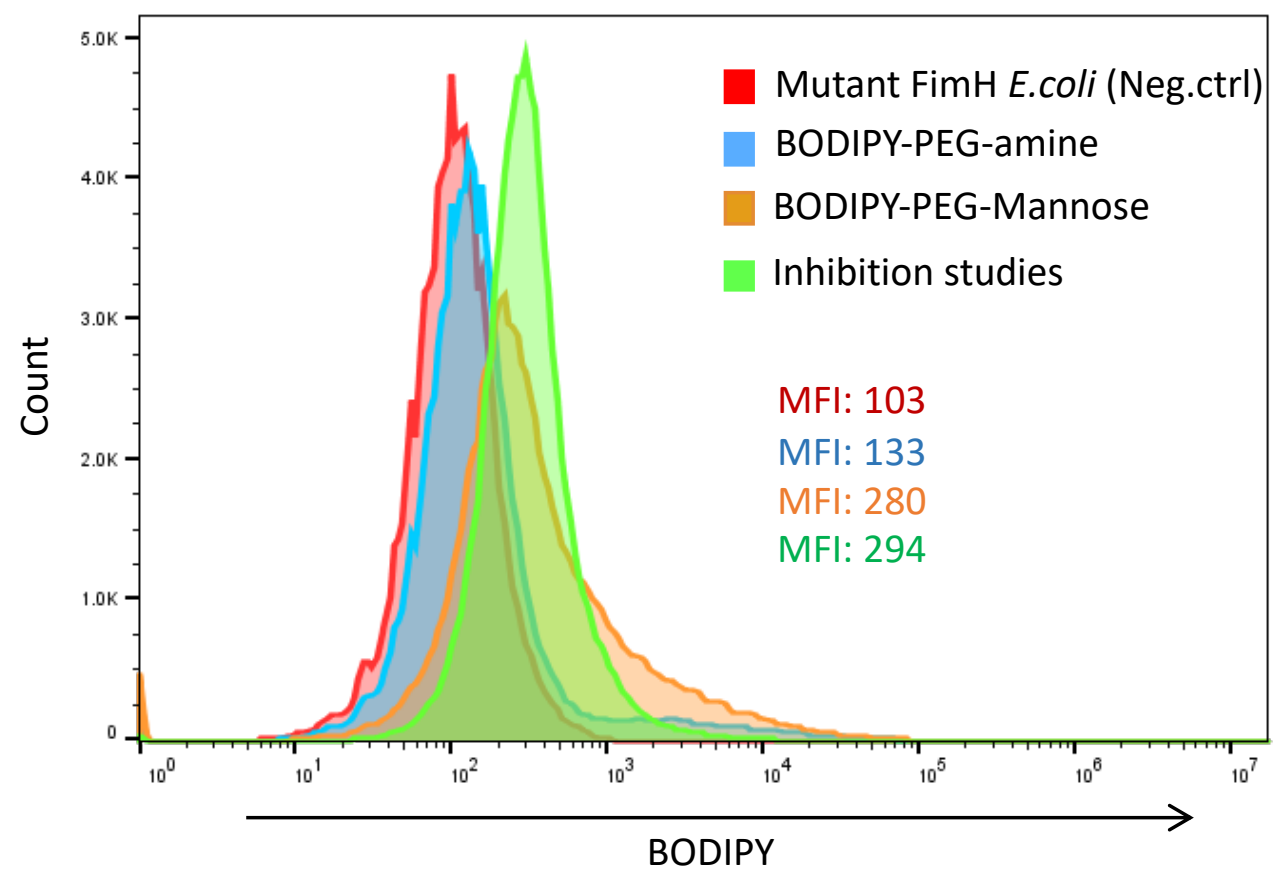

Figure 14: Inhibition binding studies to investigate the non-specific binding of BODIPY-PEG-Mannose probe to FimH mutant E.coli cells by flow cytometry. Negative control, probe absent (red). Non-specific binding of $25 \mu \mathrm{M}$ control BODIPY-PEG-amine probe to FimH mutant E.coli cells (blue). Non-specific binding of $25 \mu M$ BODIPY-PEGmannose probe to FimH mutant E.coli cells (orange). Inhibition studies with competitive ligand: 4-nitrophenyl $\alpha$-D-mannopyranoside (green). Analyses were all done with FimH mutant E.coli cells at $O D_{600}=1$. For each sample the analysis of 100,000 events were done. MFI: median fluorescence intensity.

A comparative study on the binding affinity of monosaccharide and disaccharide mannose-based probes were investigated on FimH E.coli cells. From the histogram, cells incubated with the BODIPY-PEG-mannose probe generated a 9.2-fold increase in fluorescent intensity compared to the unstained cells, whereas a $\sim 70$ fold increase was observed for the BODIPY-mannobiose probe (Figure 15). These results indicate that there is a higher binding affinity of disaccharide mannose for 
FimH lectins in comparison to monosaccharide mannose, which agrees with what has been reported in literature. ${ }^{78}$

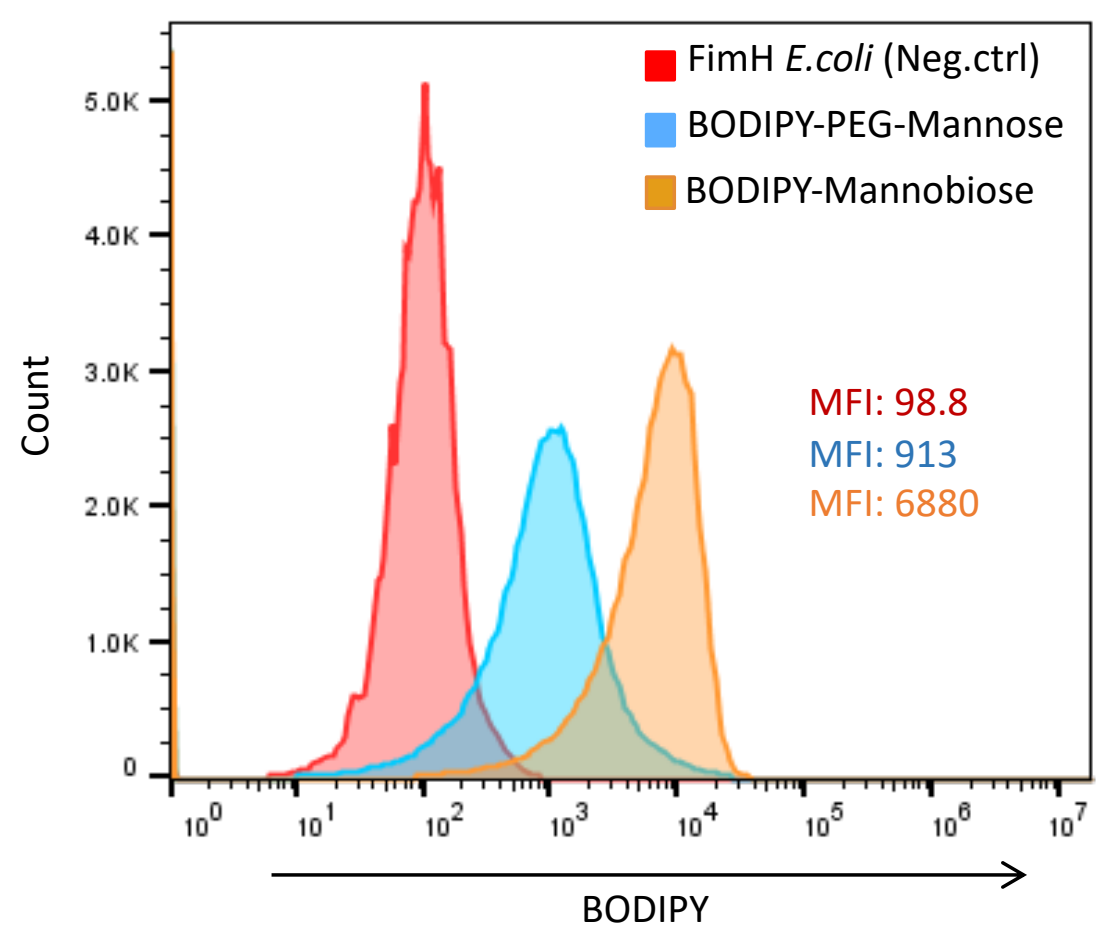

Figure 15: Binding comparison of BODIPY-PEG-Mannose and BODIPY-Mannobiose probes to FimH E.coli by flow cytometry. Negative control, probe absent (red). Binding of $0.1 \mathrm{mM}$ BODIPY-PEG-mannose probe to FimH E.coli cells (blue). Binding of $0.1 \mathrm{mM}$ BODIPY-mannobiose probe to FimH E.coli cells (orange). Analyses were all done with FimH E.coli cells at $O D_{600}=1$. For each sample the analysis of 100,000 events were done. MFI: median fluorescence intensity.

To further investigate the selectivity of the BODIPY-mannobiose probe for FimH lectins, an inhibition binding analysis was carried out, which introduced a competitive ligand. This analysis was done to investigate whether the binding of the probe to the FimH E.coli cells would be inhibited resulting in a decrease in 
fluorescence intensity. The competitive ligand selected for the analysis was a coumarin-mannobiose ligand, which consisted of the exact recognition element and linker as the BODIPY-mannobiose probe. The only difference was in their tag moiety; where for the coumarin-mannobiose competitive ligand consisted of a coumarin dye, while the BODIPY-mannobiose probe consisted of a BODIPY dye (Figure 16). Both coumarin and BODIPY dyes do not emit in the same wavelength region, thus the fluorescence intensity of the coumarin dye will not be picked up in the flow cytometry analysis. From the histogram obtained, inhibition activity was observed with the coumarin-mannobiose competitive ligand. A 4.74-fold decrease in fluorescence intensity was seen in comparison to the shift with just the BODIPYmannobiose probe (Figure 17). Similar analysis was also conducted on FimH mutant E.coli cells, resulting in no inhibition activity, instead the same fluorescence intensity shifts were observed for the BODIPY-PEG-amine, BODIPY-mannobiose probe and competitive ligand due to the "stickiness" of the probes (data not shown).

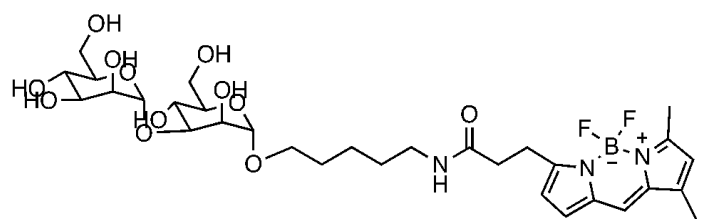

1.8 BODIPY-Mannobiose

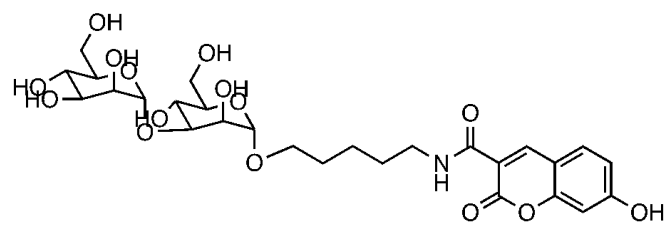

1.10 Coumarin-mannobiose

Figure 16: Chemical structure of BODIPY-Mannobiose and Coumarin-Mannobiose 


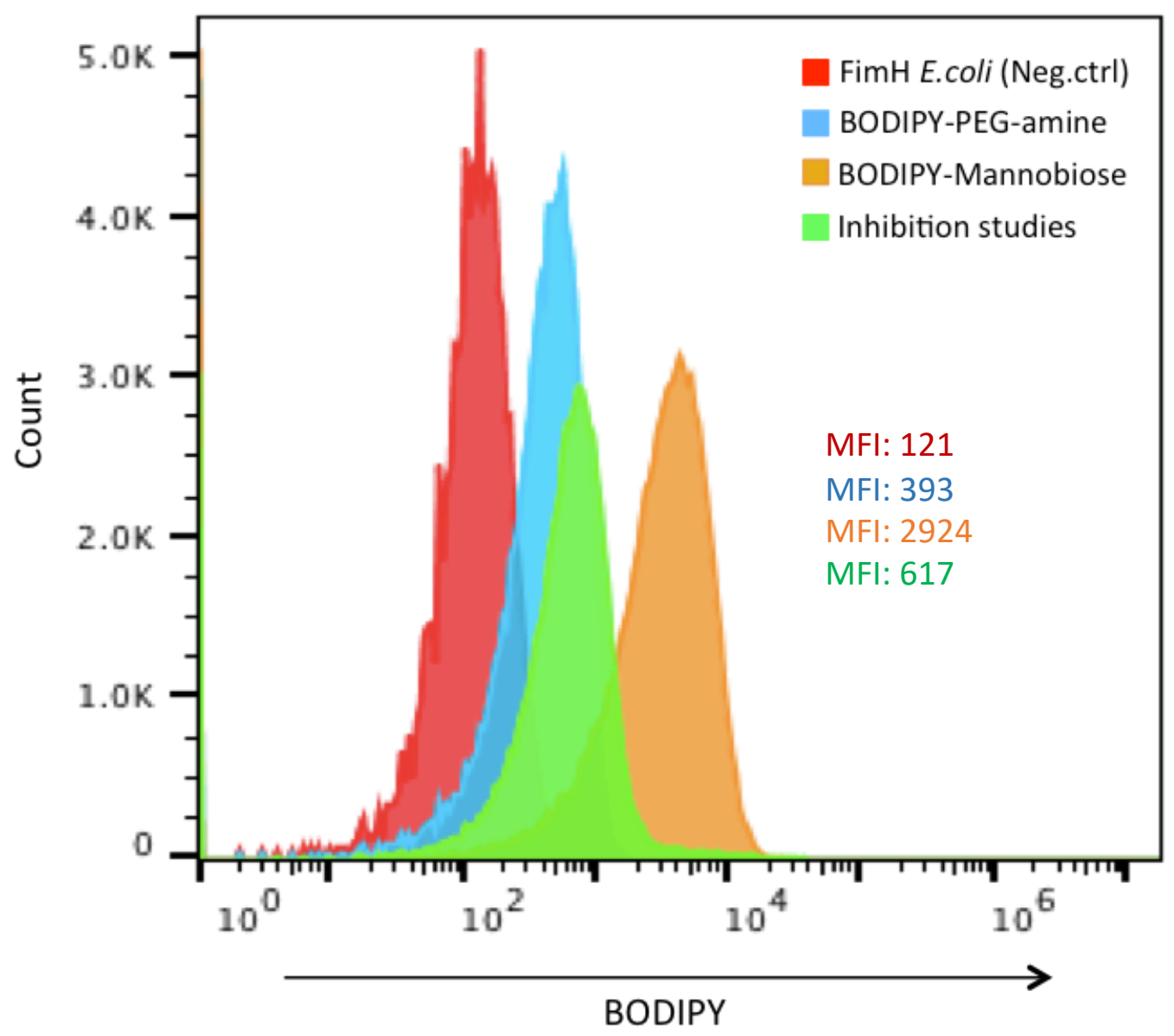

Figure 17: Inhibition binding studies to investigate the selectivity of BODIPY-

Mannobiose probe binding to FimH E.coli cells by flow cytometry. Negative control, probe absent (red). Non-specific binding of $0.1 \mu M$ control BODIPY-PEG-amine probe to FimH E.coli cells (blue). Selective binding of $0.1 \mu M$ BODIPY-PEG-mannose probe to FimH E.coli cells (orange). Inhibition of this selective binding by the competitive ligand: coumarin-mannobiose (green). Analyses were all done with FimH E.coli cells at $O D_{600}=1$. For each sample the analysis of 100,000 events were done. MFI: median fluorescence intensity. 


\subsubsection{Direct binding and inhibition binding of mannose probes on C. fimi}

Direct binding analyses were done on C.fimi cells at three different $\mathrm{OD}_{600} \approx 1.0,0.6$ and 0.3 to determine which $\mathrm{OD}_{600}$ was the most effective for conducting this experiment. The stickiness of the BODIPY-PEG component of the probe to C.fimi was also investigated with the BODIPY-PEG-amine. The histograms obtained for all three $\mathrm{OD}_{600}$ show a similar trend in fluorescence intensity shifts of the BODIPY-PEG-amine and BODIPY-PEG-mannose probe (Figure 18). The results show that the BODIPYPEG component of the probe is in fact sticky due to the $\sim 3$-fold fluorescence intensity increase that was observed with the BODIPY-PEG-amine in comparison to the unstained cells. In addition, larger fluorescence intensity shift was observed with the BODIPY-PEG-mannose probe suggesting that there was selectivity of the probe to C.fimi. From all three $\mathrm{OD}_{600}$ investigated the $\mathrm{OD}_{600} \approx 0.3$ (Figure 18 C) gave the best results due to the elimination of the shoulder peak that is observed with the C.fimi cells at $\mathrm{OD}_{600} \approx 1.0$ and 0.6 (Figure $18 \mathrm{~A}, \mathbf{B}$ ). 

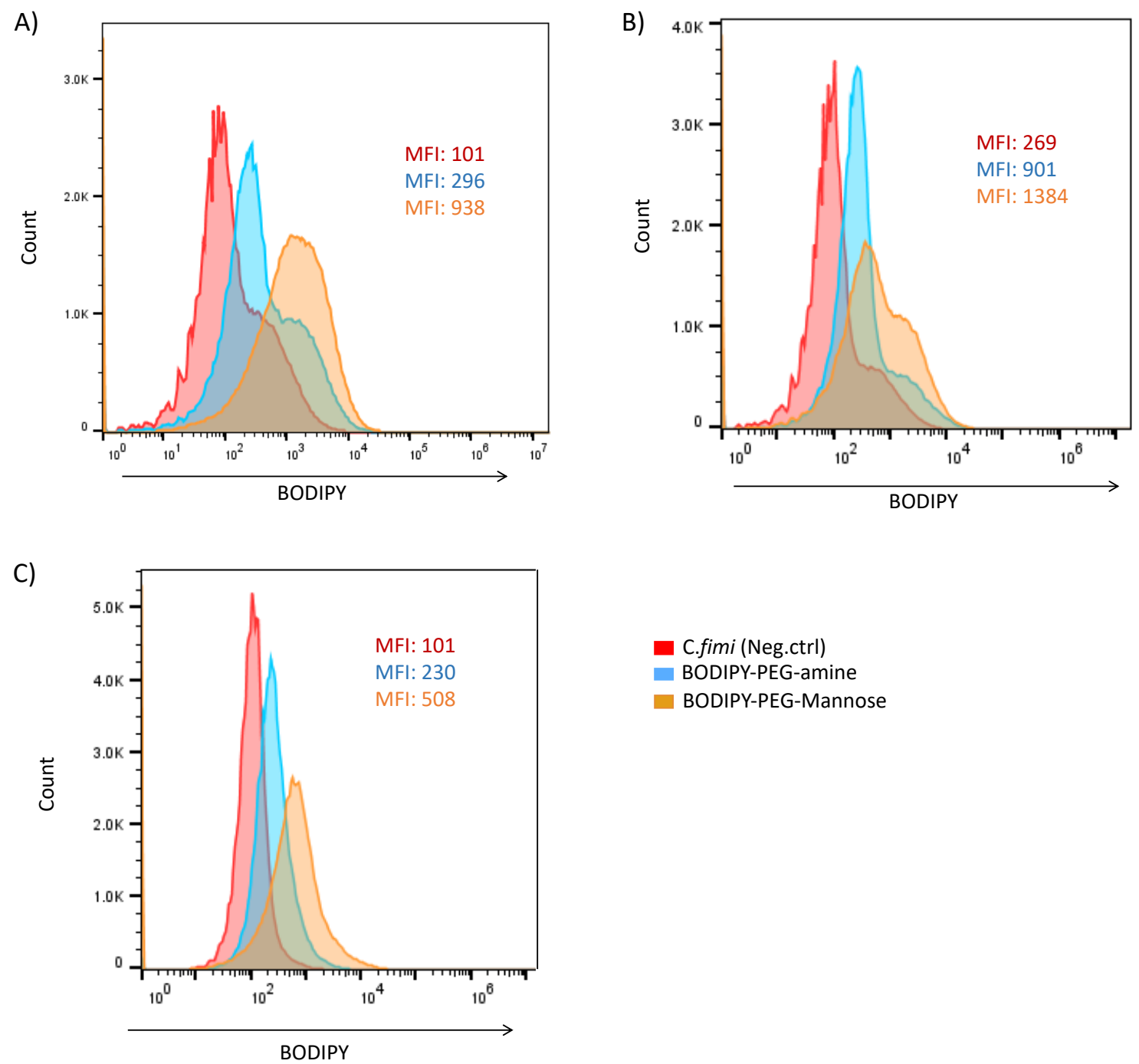

C.fimi (Neg.ctrl) BODIPY-PEG-amine BODIPY-PEG-Mannose

Figure 18: Binding performance of BODIPY-PEG-amine and BODIPY-PEG-Mannose probe to C.fimi cells by flow cytometry. The following $O D_{600}$ values were tested: $A$ ) $\left.O D_{600} \approx 1.0, B\right) O D_{600} \approx 0.6$ and C) $O D_{600} \approx 0.3$. Negative control, probe absent (red). Non-specific binding of $25 \mu \mathrm{M}$ control BODIPY-PEG-amine probe to C.fimi cells (blue). Direct binding studies of $25 \mu$ M BODIPY-PEG-mannose probe binding to C.fimi cells. For each sample the analysis of 100,000 events were done. MFI: median fluorescence intensity. 
To further investigate the selectivity of the BODIPY-PEG-mannose probe for C.fimi cells, an inhibition binding analysis was carried out with the competitive ligand, 4nitrophenyl $\alpha$-D-mannopyranoside. This analysis was done to investigate whether it will inhibit the probe-C.fimi interaction. However, from the histogram obtained, no inhibition was observed since the fluorescence intensity did not decrease by the addition of this ligand (Figure 19). Indicating that the 4-nitrophenyl $\alpha$-Dmannopyranoside was not an effective competitive ligand and therefore a ligand with stronger inhibition activity is needed.

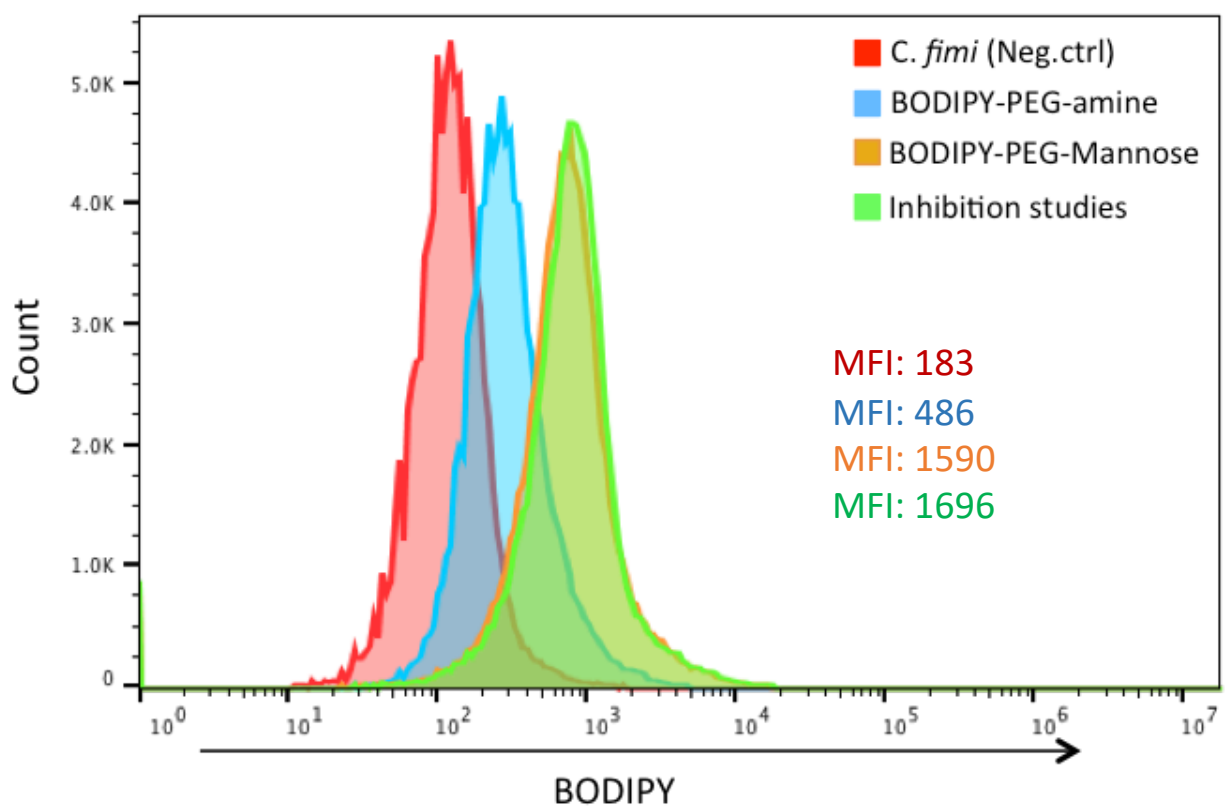

Figure 19: Investigating the selectivity of BODIPY-PEG-Mannose probe binding to C.fimi cells by flow cytometry. Negative control, probe absent (red). Non-specific binding of $0.1 \mathrm{mM}$ control BODIPY-PEG-amine probe to C.fimi cells (blue). Selective binding of $0.1 \mathrm{mM}$ BODIPY-PEG-mannose probe to C.fimi cells (orange). Inhibition of this selective binding by the competitive ligand: 4-nitrophenyl $\alpha$-D-mannopyranoside (green). For each sample the analysis of 100,000 events were done. MFI: median fluorescence intensity. 
A comparative study on the binding affinity of monosaccharide and disaccharide mannose-based probes were investigated on C.fimi cells. From the histogram, cells incubated with the BODIPY-PEG-mannose probe generated a $\sim 11.5$-fold increase in fluorescent intensity compared to the unstained cells, whereas a slightly larger fluorescence intensity shift was observed for the BODIPY-mannobiose probe (Figure 20).

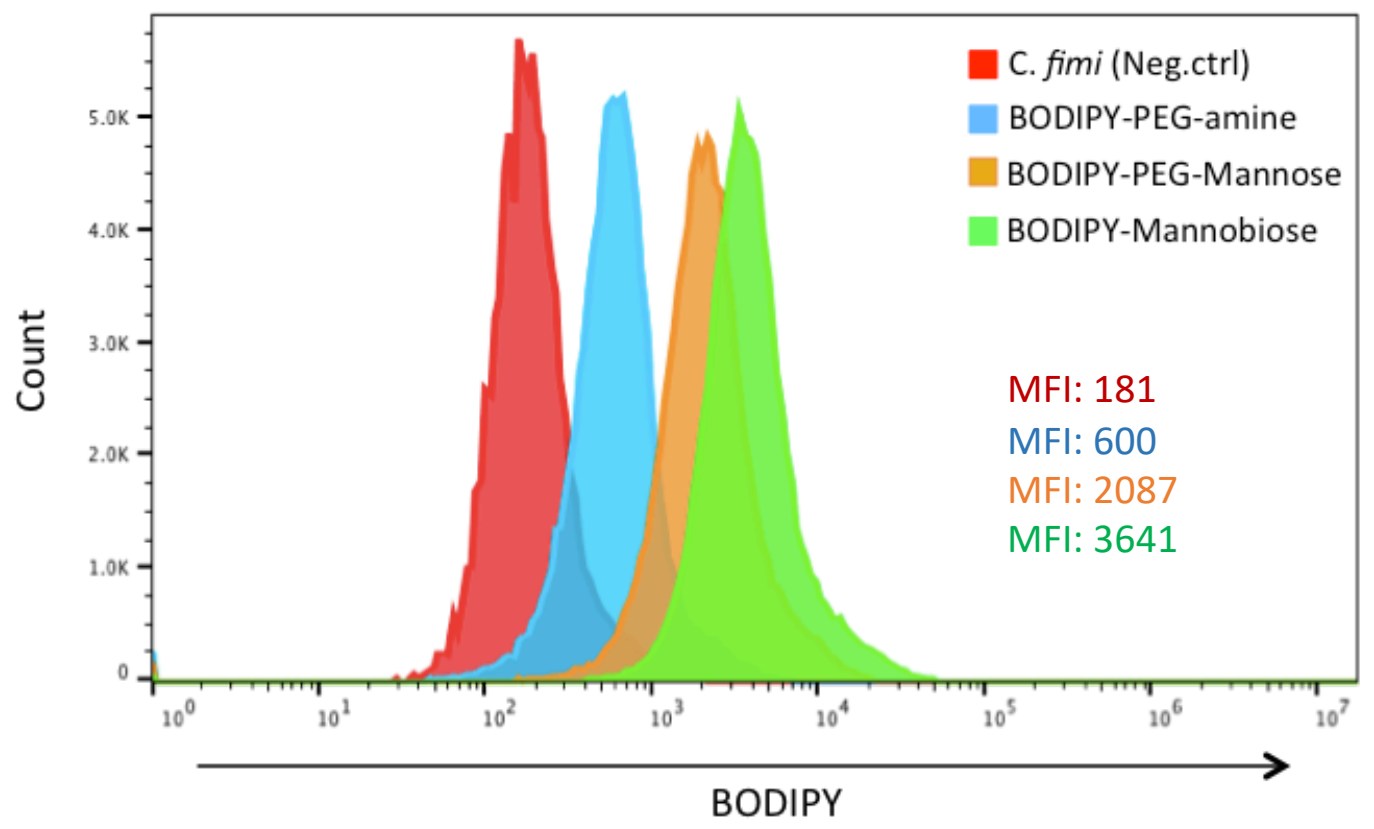

Figure 20: Binding comparison of BODIPY-PEG-Mannose and BODIPY-Mannobiose probes to C.fimi by flow cytometry. Negative control, probe absent (red). Non-specific binding of $0.1 \mathrm{mM}$ control BODIPY-PEG-amine probe to C.fimi cells (blue). Binding of 0.1 mM BODIPY-PEG-mannose probe to C.fimi cells (orange). Binding of $0.1 \mathrm{mM}$ BODIPY-mannobiose probe to C.fimi cells (green). Analyses were all done with cells at $O D_{600}=0.231$. For each sample the analysis of 100,000 events were done. MFI: median fluorescence intensity. 


\subsubsection{Direct binding and inhibition binding of mannose probes on C. flavigena}

Initially, direct binding analyses were done on C.flavigena cells at three different $\mathrm{OD}_{600} \approx 1.0,0.6$ and 0.3 to determine which $\mathrm{OD}_{600}$ was the most effective for conducting this experiment. It was determined that an $\mathrm{OD}_{600}$ close to 0.3 was optimal, which is similar to the results observed for C.fimi cells described above. To investigate the selectivity of the BODIPY-PEG-mannose probe for C.flavigena cells, both a direct and inhibition binding analysis were carried out. The competitive ligand selected for the analysis was the 4-nitrophenyl $\alpha$-Dmannopyranoside. From the histogram obtained, a fluorescence intensity shift was observed with the BODIPY-PEG-mannose probe from the direct binding analysis suggesting that there was selectivity of the probe to C.flavigina. For the inhibition studies, a slight inhibition activity can be seen with a slight decrease in fluorescence intensity compared to BODIPY-PEG-mannose (Figure 21). However, a more effective competitive ligand with stronger inhibition activity is needed. 


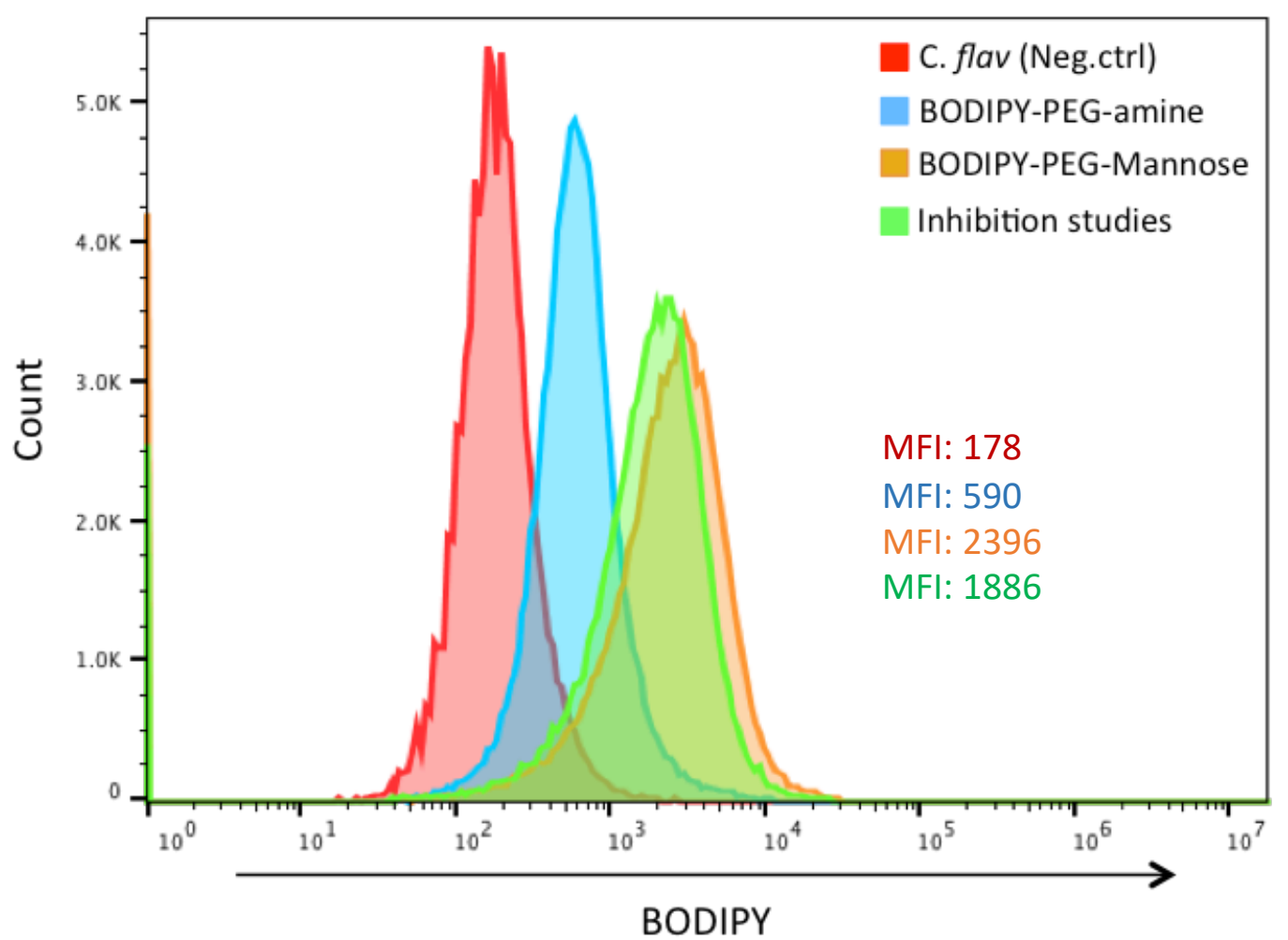

Figure 21: Investigating the selectivity of BODIPY-PEG-Mannose probe binding to C.flavigena cells by flow cytometry. Negative control, probe absent (red). Non-specific binding of $0.1 \mathrm{mM}$ control BODIPY-PEG-amine probe to C.flavigena cells (blue). Selective binding of $0.1 \mathrm{mM}$ BODIPY-PEG-mannose probe to C.flavigena cells (orange). Inhibition of this selective binding by the competitive ligand: 4-nitrophenyl $\alpha-D$ mannopyranoside (green). For each sample the analysis of 100,000 events were done. MFI: median fluorescence intensity.

A comparative study on the binding affinity of monosaccharide and disaccharide mannose-based probes were investigated on C.flavigena cells. From the histogram results, cells incubated with the BODIPY-PEG-mannose probe and BODIPYmannobiose probe generated a slight difference in fluorescent intensity compared 
to the unstained cells, with a 10-fold increase for BODIPY-PEG-mannose and a 13.5-fold increase for BODIPY-mannobiose. (Figure 22).

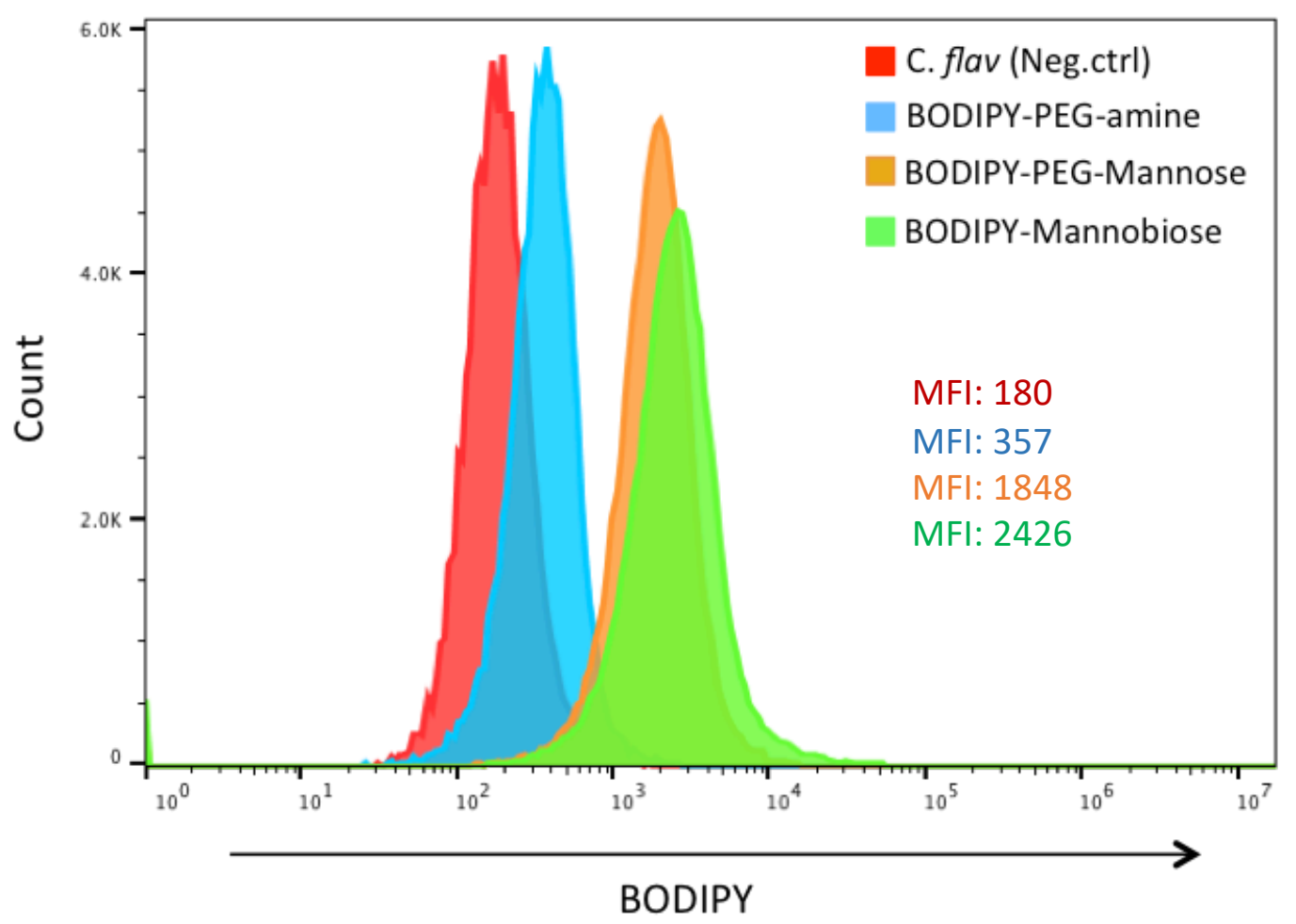

Figure 22: Binding comparison of BODIPY-PEG-Mannose and BODIPY-Mannobiose probes to C.flavigena by flow cytometry. Negative control, probe absent (red). Nonspecific binding of $0.1 \mathrm{mM}$ control BODIPY-PEG-amine probe to C.flavigena cells (blue). Binding of $0.1 \mathrm{mM}$ BODIPY-PEG-mannose probe to C.flavigena cells (orange). Binding of 0.1 mM BODIPY-mannobiose probe to C.flavigena cells (green). Analyses were all done with cells at $O D_{600}=0.234$. For each sample the analysis of 100,000 events were done. MFI: median fluorescence intensity.

\subsubsection{Fluorescence Microscopy}

Fluorescence microscopy was used in order to test the binding specificity of the mannose-based probes for mannose-binding lectins. The probes were initially 
tested on the control FimH E.coli cells (NRG857c), where BODIPY-mannose probes were incubated with cells at $\mathrm{OD}_{600}=1.00$ and fixed onto glass slides with $2 \% \mathrm{PFA}$ and co-stained cells with a blue-fluorescent DAPI nucleic acid stain. The images were generated in different channels: bright field channel, fluorescence channels (DAPI and BODIPY) and a merged channel, which merged the bright field and fluorescence channels into a single image. A $6 \times 10^{-3} \mathrm{mM}$ probe concentration was optimal for the fluorescence microscopy studies, which clearly showed specific binding of BDP-PEG-mannose probes for the FimH E.coli cells as illustrated in Figure 23. However, it was also observed that not all the cells were stained with the probe, which might be due to cells not having the exact distribution of FimH lectins on their surfaces.

1

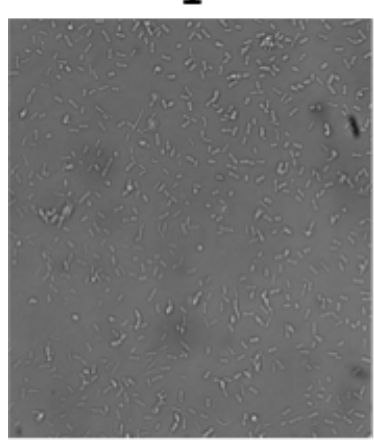

2

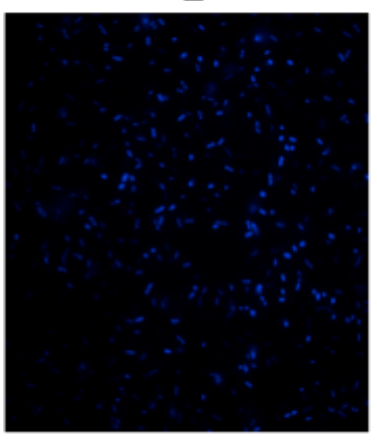

3

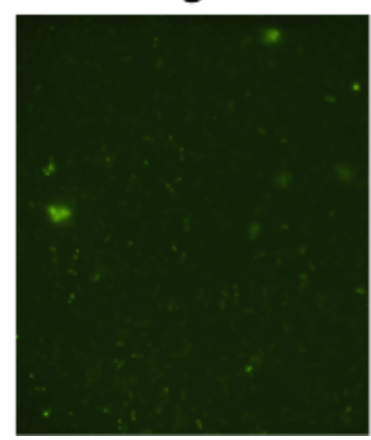

4

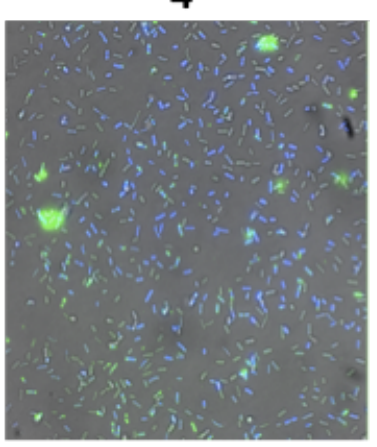

Figure 23: FimH E.coli Microscopy images for co-localization investigation with BODIPY-PEG-mannose probe. FimH E.coli (NRG857c) at $O D_{600}=1.00$ incubated with 6 $x 10^{-3} \mathrm{mM}$ of BODIPY-PEG-mannose probe. Channels: 1) Bright field, 2) DAPI, 3) BODIPY and 4) Merged. 
Fluorescence microscopy was also used to visualize whether putative mannosebinding lectins were present on C.fimi and C.flavigena cells. Cells at $\mathrm{OD}_{600} \approx 0.23$ were incubated with probes and prepared via an agarose pad method outlined in section 2.8. The agarose pad method was used as it effectively immobilized cells during imaging and minimized the overlapping of cells. Cells were also co-stained with a DAPI stain and instead of chemical fixation with PFA, cells were treated with $1 \mathrm{mM}$ of $\mathrm{NaN}_{3}$. A probe concentration of $25 \times 10^{-2} \mathrm{mM}$ was optimal for the fluorescence microscopy studies, which showed some binding of probes to cells as illustrates in Figure 24.

A)

1

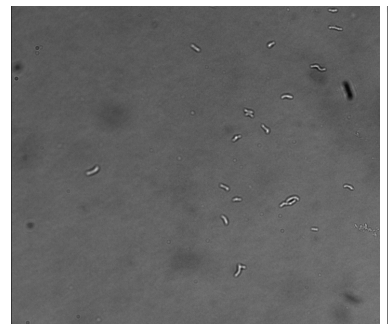

B)

1

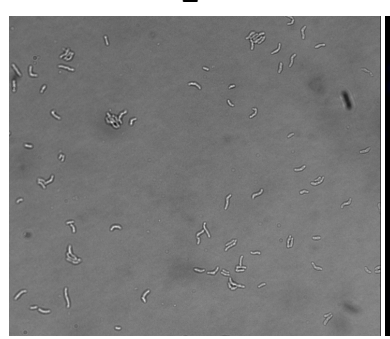

2
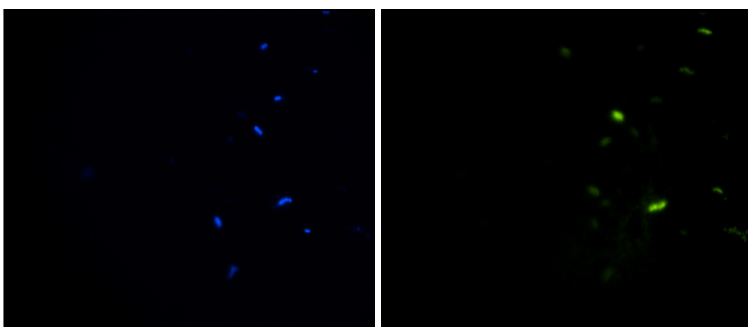

3

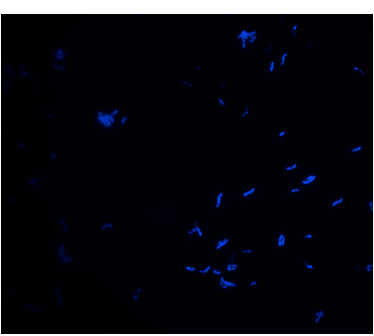

3

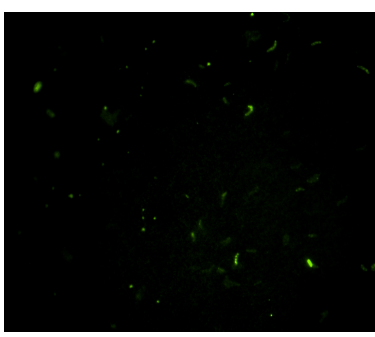

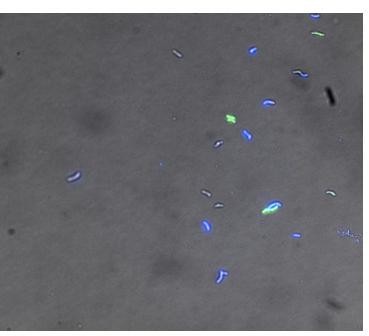

4

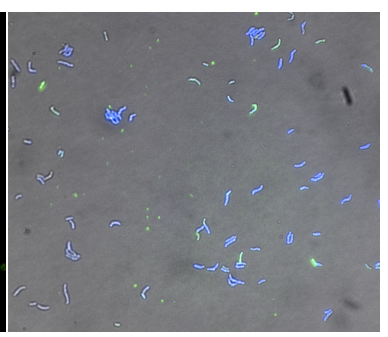

Figure 24: C.fimi Microscopy images for co-localization investigation. A) Cells incubated with $25 \times 10^{-2} \mathrm{mM}$ of BODIPY-PEG-mannose probe. B) Cells incubated with $25 \times 10^{-2} \mathrm{mM}$ of BODIPY-mannobiose probe. Studies were done with cells at $O D_{600}=$ 0.231. Channels: 1) Bright field, 2) DAPI, 3) BODIPY and 4) Merged. 


\subsection{Discussion}

\subsubsection{Development of the mannose-based probes}

For the development of mannose-based probes the selection of the probe composition (tag, linker, and recognition element) were critical for their design, as it will have an effect on their overall efficiency. Three mannose-based probes were developed; two of them consisted of a monosaccharide mannose recognition element and PEG linker, while the third probe consisted of a disaccharide mannose recognition element and a pentylamine linker. All three probes were comprised of a BODIPY dye as the tag moiety, functionalized with either an alkyne or NHS ester group which allowed for the conjugation of the dye to the probe to occur through the $\mathrm{CuAAc}$ or succinimidyl ester reactions, respectively. For the most part, the synthesis of the monosaccharide and disaccharide mannose probes did not pose any issues, with most of them being obtained at high product yields of $80 \%$ or more.

For the development of the monosaccharide mannose probes, two BODIPY derivatives were tested as the tag moiety. Comparing the two derivatives, issues were observed with the planar BODIPY-alkyne (1.2) derivative. The planar BODIPYalkyne (1.2) tag gave a much lower product yield of $44.8 \%$ compared to the BODIPY-alkyne (1.1) tag, which gave about a 95\% relative percent yield via CuAAc reaction. A reason for why labelling differences were observed is due to the steric and electronic differences between the two BODIPY derivatives (1.1 and 1.2). Particularly since one of the planar BODIPY (1.2) dye is directly conjugated to the PEG linker of the probe while the other is not. In addition, because the BODIPY- 
alkyne (1.1) consists of a propylamide-based spacer it might be enhancing the aqueous solubility during the CuAAc reaction. While the planar BODIPY-alkyne (1.2) was observed to experience solubility issues during the CuAAc reaction, requiring $\sim 30 \%$ excess of the solvent (DCM) in order to keep it in solution. Also the alkyne group might be unstable located at the C-2 position of the planar BODIPY (1.2) molecule, which might be favouring its degradation during the CuAAc reaction.

The linker components selected for the development of the probes have shown to offset the hydrophobic properties of the BODIPY dyes, and thus increasing its solubility in aqueous environments. As well as, minimizing the interactions between the recognition elements (monosaccharide and disaccharide mannose) and the BODIPY component so that there is no interference between their separate function, which was evident from the flow cytometry and fluorescence microscopy results obtained for the cells analyzed.

\subsubsection{Functionality of mannose-based probes on FimH E.coli}

In this research, the FimH E.coli strain NRG857c was used as control cells since their surfaces are decorated with the well-known mannose-binding FimH lectins. In addition to this strain, a second strain NRG857c $\Delta$ FimH was used where its FimH lectin was deleted from the genome. The use of these cells were essential as it allowed for the study of the selectivity of these mannose-based probes using flow cytometry and fluorescence microscopy techniques. Alongside testing the selectivity of the mannose-based probes for FimH lectins, it was also necessary to make sure 
that the tag moiety and the linker components of the probe did not contribute significantly to non-specific interactions of the probes with the cells. In order to investigate this, cells were incubated with a BODIPY-PEG-amine compound missing the mannose recognition element. Ideally, this compound should not interact with the FimH lectins. However a distinctive non-specific binding of this compound to both the FimH and FimH mutant E.coli cells were observed. This distinctive nonspecific binding was also observed with the FimH mutant E.coli cells incubated with the BODIPY-PEG-mannose probe, suggesting that the BODIPY-PEG component of the probe is in fact 'sticky'. This 'stickiness' observed is most likely might be occurring due to the hydrophobic interactions between the aromatic portion of the BODIPY tag and the cell surface components. However this non-specific binding observed seems to be minimal, as it was not seen to contribute to background labelling in fluorescence microscopy. Particularly, since no staining was detected in FimH E.coli cells incubated with the BODIPY-PEG-amine compound (data not shown).

Furthermore, the BODIPY-PEG-amine compound was also used to investigate the selectivity of the mannose based probes, along with inhibition-binding studies. It was evident that the mannose-based probes were specifically binding to the FimH lectins, due to the distinguishable fluorescence intensity shifts observed in the flow cytometry analyses. Additionally, binding of probes to cells was also detected by fluorescence microscopy as bright staining was seen from cells. An interesting point to note about the microscopy results obtained was that, not all the cells were stained with the probe, which might be due to the fact that not all the cells have the same distribution of FimH lectins. 
The BODIPY-mannobiose probe, consisting of the disaccharide $3 \alpha$-mannobiose as the recognition element, increased the binding affinity to FimH lectins in comparison to the BODIPY-PEG-mannose probe, consisting of a monosaccharide mannose as the recognition element. FimH E.coli cells incubated with the BODIPYPEG-mannose probe generated a 9.2-fold increase in fluorescent intensity compared to the unstained cells, whereas a $\sim 70$-fold increase was observed for the BODIPY-mannobiose probe, agreeing with what has been reported in literature. ${ }^{78}$ Furthermore, inhibition binding studies with the competitive ligands, 4-nitrophenyl $\alpha$-D-mannopyranoside and coumarin-mannobiose, confirmed the selectivity of the mannose-based probes for FimH lectins by successfully inhibiting the lectin-probe interactions observed, as predicted.

\subsubsection{Discovery of mannose-binding lectins on C. fimi and C. flavigena}

Due to the promising results obtained with these mannose-based probes on FimH E.coli, it gave us the confidence to move on to using these probes as analytical tools to detect the existence of mannose-binding lectins on microorganisms. Since C.fimi and C.flavigena have through genome sequencing recently been suggested to containing a putative mannose-binding lectin ${ }^{66}$, we used these probes to explore whether they are applicable to reveal the presence of mannose-binding lectins on their surfaces.

Exploratory work was performed in order to optimize the conditions for binding of the mannose-based probes to the surfaces of $C$.fimi and C.flavigena_cells. 
Unlike the FimH E.coli cells described above, it was a bit challenging to determine what optical density at $\lambda 600 \mathrm{~nm}\left(\mathrm{OD}_{600}\right)$ was optimal for flow cytometry analysis. From all the $\mathrm{OD}_{600}$ values investigated the $\mathrm{OD}_{600}$ between 0.23 to 0.3 gave the best results due to the elimination of the 'shoulder' peak observed with both the C.fimi and C.flavigena cells at $\mathrm{OD}_{600}$ values greater than 0.6 . This observed peak 'shouldering' might be due to the formation of cell clumps at higher concentrations thus generating the different cell populations seen in the histograms for both the unstained and stained cells.

Consequently, the direct binding and inhibition binding analyses were performed at the optimal $\mathrm{OD}_{600}$ values for both $C$.fimi and $C$.flavigena cells to search for these putative mannose-binding lectins on their surfaces. The direct binding results obtained suggested that the probes were somewhat binding to the cells due to the distinct fluorescence intensity shift observed on their flow cytometry histograms. Additionally, binding of probes to cells was also detected by fluorescence microscopy, as bright staining was seen for some cells. Comparing the monosaccharide and disaccharide probes, a significant difference in their binding affinities for the cells were not observed. Since its known that multivalency significantly enhances binding 29 , it was intriguing that a greater shift in fluorescence intensity was not obtained with the disaccharide probe. Assuming that mannose-binding lectins actually exist on the surfaces of these cells, it might be that these lectins prefer disaccharides comprising of specific glycosidic linkages. The BODIPY-mannobiose probe consists of a $3 \alpha$-mannobiose as the recognition element, which is composed of two mannose residues joined by a $\alpha-1,3$ glycosidic linkage. 
To corroborate the binding results obtained with the direct binding studies, an inhibition binding studies with the competitive ligand, 4-nitrophenyl $\alpha$-Dmannopyranoside was performed. The results obtained were inconclusive as the inhibition activities observed were slim to none. Indicating that the 4-nitrophenyl $\alpha$ D-mannopyranoside was not an effective competitive ligand, as it was not observed to compete with the probe, which was evident from the flow cytometry histograms where a decrease in fluorescence intensity was not observed. Thus, these studies could not validate whether the probes were actually binding to mannose-binding lectins. 


\subsection{Concluding Remarks}

The presence of mannose-binding lectins has been speculated to play important biological roles in cellular adhesion, cell differentiation, pathogen recognition and defense against pathogenic microorganisms. ${ }^{20,19}$ The search for the development of effective techniques to probe them has been an ongoing research interest. This chapter looked at the development of mannose-based chemical probes to explore whether mannose-binding lectins exist on the surfaces of microorganisms. A fast and efficient synthesis of BODIPY-mannose probes were presented and the binding affinities of monosaccharide and disaccharide mannose recognition elements were compared. The binding of these probes to the control FimH E.coli cells demonstrated, that these probes could reasonably detect mannose-binding lectins on cell surfaces. These probes have also allowed for the use of flow cytometry and fluorescence microscopy techniques to efficiently detect mannose-binding lectins. However, it should be mentioned that for the investigation of putative mannosebinding lectins on the surfaces of C.fimi and C.flavigena the results obtained were inconclusive. Therefore, additional steps are required to clarify these results.

\subsubsection{Future Work}

Future work on this project involves the clarification of the results obtained for C.fimi and C.flavigena in order to confirm whether putative mannose-binding lectins exist on their surfaces. To achieve this, certain protocols and techniques used in this project need to be optimized. For instance, different competitive ligands need to be 
investigated for the inhibition binding studies using flow cytometry technique.

Other techniques such as carbohydrate affinity chromatography can be employed, in order to support the results generated. Future work will also look into modifying the current mannose-based probes with different tag, linker, and mannose recognition elements, in order to enhance its efficiency for detecting mannosebinding lectins in various microorganisms. For instance, photoreactive linkers can be used as they could covalently link weakly bound lectins to the probe. ${ }^{18}$ Recognition elements comprised of multivalent mannose ligands will also be looked into, since multivalent interactions greatly enhance the binding affinity and selectivity. ${ }^{19}$. It would be interesting to explore the binding profiles of other mannobiose recognition elements with different glycosidic linkages than the one used in this thesis such as $2 \alpha$-mannobiose and $6 \alpha$-mannobiose, which are composed of two mannose residues joined by a $\alpha-1,2$ and a $\alpha-1,6$ glycosidic linkage, respectively. 


\section{Chapter 2: Development of Sialic Acid-based Fluorescent Probes for Enzyme Studies}

\subsection{Introduction}

\subsubsection{Overview of Sialoglycans}

Sialic acids, are a negatively charged nine-carbon monosaccharide family, typically found in the terminated ends of oligosaccharide chains in cell surface glycoproteins and glycolipids. ${ }^{34,35}$ The family of sialic acids currently comprise of more than 50 structurally different natural analogues (Figure 25). ${ }^{35}$ The largest structural variations occur at the carbon $5(\mathrm{C}-5)$ position of sialic acids, where the modifications at this position determines the core sialic acid forms which are $\mathrm{N}$ acetylneuraminic acid (Neu5Ac), N-glycolylneuraminic acid (NeuGc), and 2-keto-3deoxynononic acid (KDN). ${ }^{36}$ Neu5Ac is the most abundant and widely synthesized member of the family, with its structure comprising of a 6 carbon carboxylic acid ring with an acetamido group at the C-5 position and hydroxyl groups at C-4, C-7, C8, and C-9. ${ }^{37}$ Neu5Gc are common amongst most mammalian tissues but conspicuously absent on human cells, due the deletion of the hydroxylase (CMAH) gene, which is vital for its formation. ${ }^{37,38}$ Neu5Gc is structurally similar to Neu5Ac, except for the hydroxyl group on the N-5 acetyl moiety. KDN on the other hand constitutes of a hydroxyl group at the C-5 position. Although KDN is present amongst mammalian cells they are often expressed at lower levels in comparison to the other sialic acid members. ${ }^{39}$ Further structural diversity of these core sialic acid members can be generated by modification of their hydroxyl groups at C-4, C-7, C-8, 
and C-9 with 0-acetyl, O-methyl, O-sulfate, O-lactyl, or phosphate substituents. ${ }^{36,40 \text {, }}$ ${ }^{41}$ Besides their structural diversity, they are notably diverse in their glycosidic linkages, fundamental glycan chains and their exposed location. ${ }^{42}$ Given their remarkable diversity, it is not surprising that they are involved in several biological roles, including regulation of solubility, stability and half-lives of secreted proteins, act as cellular receptors for influenza viruses, and ligands during cell-cell interactions and cell-molecule recognition. ${ }^{41,42,43}$ For instance, non-sialylated glycoforms have been shown to exhibit drastically shorter in-vivo half-lives compared to their sialylated equivalents. ${ }^{44}$ Increasing the amount of sialic acid residues of therapeutic glycoprotein boosts in-vivo half-life. ${ }^{44}$

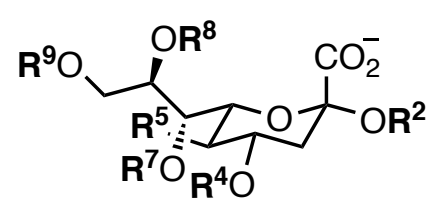

Figure 25: The general chemical structure of sialic acid. Various structural of modifications can occur at the $R$ positions.

\subsubsection{Types of Sialoglycans}

Sialylation is defined as the modification of proteins or lipids, in which sialic acid moieties are introduced onto the terminal end of an oligosaccharide chain in a glycoprotein. Two of the most commonly studied glycoforms in protein sialylation are N-linked glycans and 0- linked glycans. The presences of these glycans are 
significant in promoting biological activity in a variety of different glycoproteins. Nlinked protein glycosylation is a co-translational event that occurs in the endoplasmic reticulum during protein synthesis. ${ }^{45}$ In the endoplasmic reticulum, $\mathrm{N}$ glycosidic linkages are known to promote the proper folding of newly synthesized polypeptides. ${ }^{46}$ On the other hand, 0 -linked protein glycosylation occurs after the translational event in the Golgi apparatus. ${ }^{45}$ Before glycans reach the cell surface, they are further modified and developed in the Golgi apparatus, in order to produce mature glycoproteins. ${ }^{45}$

N-linked glycans are linked to the amide nitrogen of an asparagine (Asn) residue. ${ }^{40,45}$ In terms of structure, all N-linked glycans share a common core carbohydrate sequence; two $\mathrm{N}$-acetylglucosamine (GlcNAc) residues linked to a branched mannose (Man) trio $\left(\mathrm{Man}_{3}-\mathrm{GlcNAc}_{2}-\mathrm{N}-\mathrm{Asn}\right)$ illustrated in Figure 26. ${ }^{47}$ Several carbohydrate residues may also be linked onto the Man trio of the core, forming large and branched oligosaccharide chains. N-linked glycans can be classified as high-mannose, hybrid, or complex types. ${ }^{47}$ Typically, the hybrid and complex subgroups often contain sialic acid residues. ${ }^{47}$ 


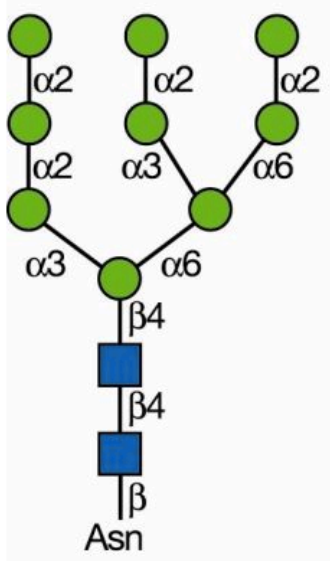

Oligomannose

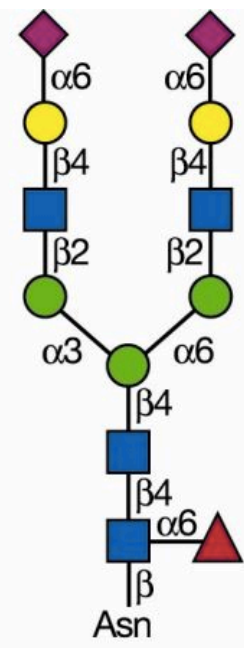

Complex

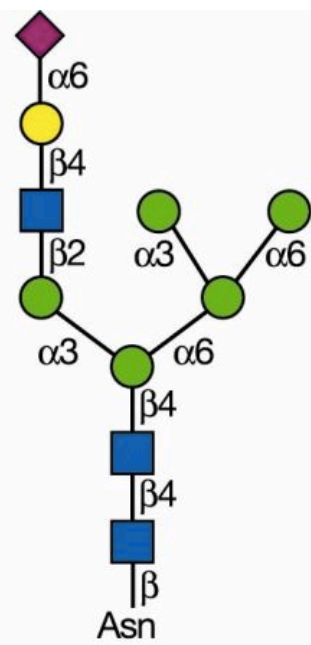

Hybrid

$\begin{array}{ll}\square \text { N-acetylglucosamine } & \bigcirc \text { Galactose } \\ \square \text { N-acetylgalactosamine } & \bigcirc \text { Mannose } \\ \triangle \text { Fucose } & \bigcirc \text { Neu5Ac }\end{array}$

Figure 26: Common core carbohydrate sequences of N-linked glycan structures. ${ }^{40}$

O-linked glycans are linked to the hydroxyl groups of serine or threonine residues (Ser/Thr). ${ }^{40,45}$ Unlike N-linked glycans, O-linked glycans do not have a preconstructed 'core' structure; instead a sequential addition of monosaccharide residues are added in a stepwise manner. ${ }^{47}$ The consecutive construction of the glycan chain only transpires after the monosaccharide $\mathrm{N}$-acetylgalactosamine GalNAc is linked to the Ser/Thr residues, catalyzed by GalNAc transferase. ${ }^{40,45}$ This GalNAc-O-Ser/Thr linkage is referred to as the Tn antigen. There are seven 0-glycan core subtypes that can all be terminated with sialic acid residues. ${ }^{40}$ The most common types are Core-1 0-glycan (Galß1-3GalNAc-O-Ser/Thr) also referred to as T antigen, Core-2 0-glycan (GlcNAc $\beta 1-6$ (Gal $\beta 1$-3)GalNAc-O-Ser/Thr), and Core-3 0glycan (GlcNAc $\beta 1$-3GalNAc-O-Ser/Thr) (Figure 27). ${ }^{40,48}$ The construction of the 
oligosaccharide chains in 0-glycans can consist of a variability of monosaccharides, branches, linkages and isomers, which typically are not too complex compared to Nglycans. ${ }^{49}$

\subsubsection{Sialyltransferases}

Sialylation of glycoproteins are catalyzed by regio-selective sialyltransferases. They are disulfide-containing, type II transmembrane glycoproteins involved in the final steps of sialic acid pathway. They catalyze the transfer of an activated sialic acid donor onto terminal galactose (Gal), GalNAc or sialic acid residues of glycoproteins through specific glycosidic linkages. ${ }^{50,51}$ The activated sialic acid donor used is a cytidine 5'-monophosphate (CMP)-Neu5Ac. In regards to their regio-selectivity and acceptor specificity, all eukaryotic sialyltransferases belong to the glycosyltransferase family 29 according to Carbohydrate-Active enZYmes (CAZy) database. ${ }^{52}$ These sialyltransferases are categorized in four subfamilies: ST3 $\beta$ galactoside $\alpha$-2,3-sialyltransferases (ST3Gal), ST6 $\beta$-galactoside $\alpha$-2,6sialyltransferase (ST6Gal), $\alpha$-N-acetylgalactosaminide $\alpha$-2,6-sialyltransferase (ST6GalNAc) and $\alpha$-N-acetylneuraminate $\alpha$-2,8-sialyltransferase (ST8Sia). ${ }^{52,53}$ They have been named according to their monosaccharide acceptor and their glycosidic linkages. ${ }^{52,53}$ These enzymes catalyze the formation of different glycosidic linkages, $\alpha-2,3$ or $\alpha-2,6$ on terminal Gal residues in N- or 0 - linked glycans; $\alpha-2,6$ on terminal GalNAc residues in 0-linked glycans and $\alpha-2,8$ on terminal Neu5Ac residues in N- or O-linked glycans. ${ }^{54}$ 


\subsubsection{ST3Gal1 vs. ST6Gal1}

ST3Gal1 and ST6Gal1 are among the few sialyltransferases that have their X-ray crystal structures elucidated. There structures have been shown to contain the glycosyltransferases GT-A (variant 2) fold. ${ }^{55,56}$ For both enzymes, the 7 core $\beta$ strands occupy similar regions, containing the conserved sialylmotifs. ${ }^{55}$ However they distinguish between their last $\beta$-strand, where in ST6Gal1 a $\alpha$-helical linker is present, which allows for the antiparallel orientation at the end of the $\beta$-sheet while in the ST3Gal1, the last $\beta$-strand is in a parallel arrangement. ${ }^{55}$, 56 In addition, ST6Gal1 catalytic domain comprises of a large N-terminal extension, which is absent in ST3Gal1, suggesting that it may be involved in substrate binding. ${ }^{55}$ Both ST3Gal1 and ST6Gal1, catalyze the transfer of Neu5Ac through the formation of $\alpha-2,3-$ and $\alpha$ 2, 6-glycosidic linkages, respectively. The ST3Gal1 enzyme recognizes the terminal Gal residue on 0-linked glycans (T antigen) forming a sialyl-T antigen (Figure 27). Whereas, the ST6Gal1 enzyme recognizes the terminal Gal residue on N-linked glycans (Figure 26). 


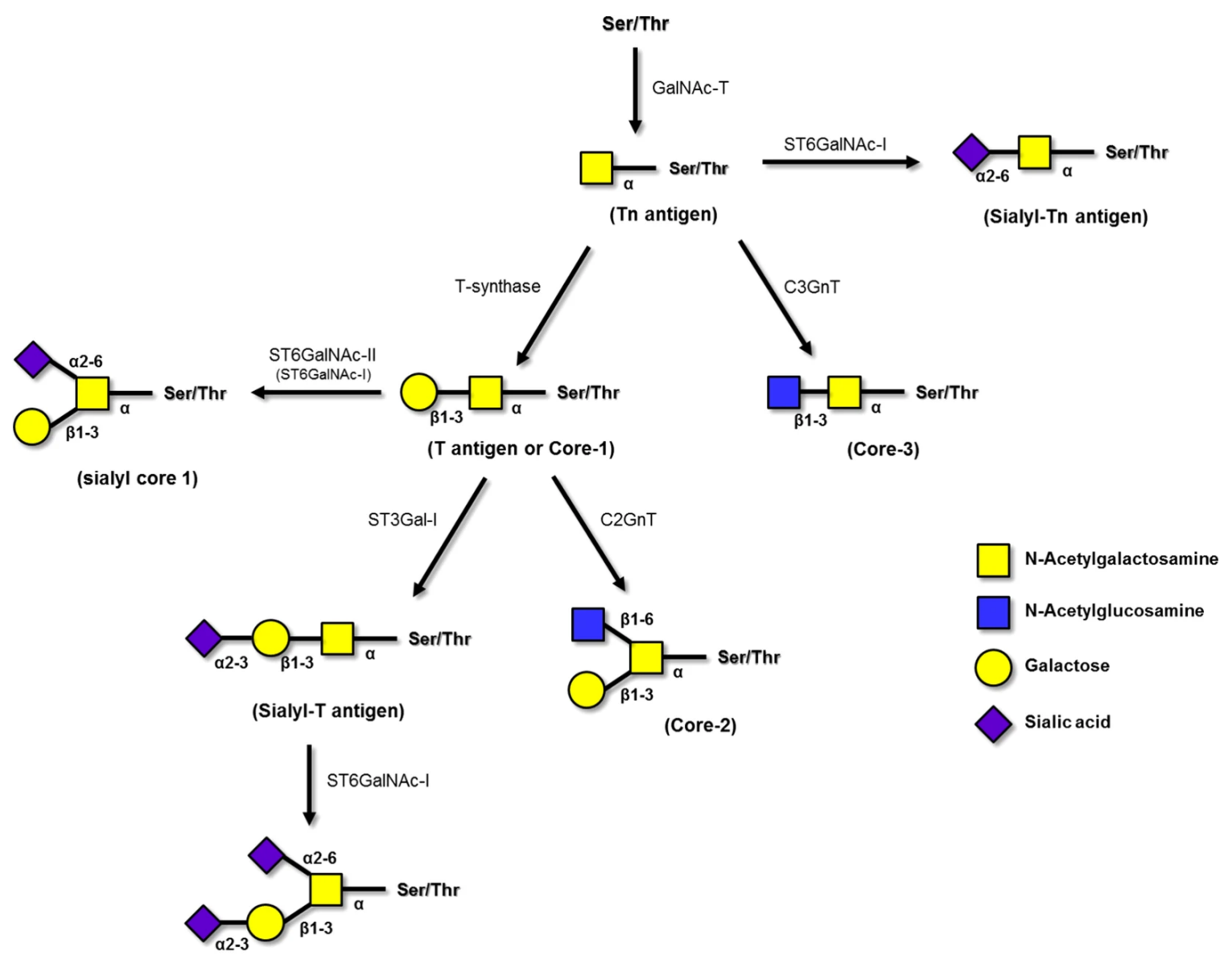

(di-sialyl core 1)

Figure 27: Biosynthesis of O-glycan chains. Illustration of Core-1 (Tn antigen), Core-2 (T antigen) and Core 3 subtype formations. Modifications of Th antigen and T antigens by ST6GalNAc1-2 and ST3Gal1 generating sialyl-Tn antigen and sialyl-T antigens. ${ }^{48}$

\subsubsection{Probing Sialylation}

Since sialylation is typically the final step of glycan modifications, the level of sialylation reflects the maturation of glycosylation. Manipulation and control of sialylation levels have been an important research interest, particularly in the development of therapeutic proteins. Therefore, developing simple methods for 
probing sialylation will be beneficial for monitoring glycosylation as well as understanding its biological effects on protein function.

Traditionally, the uses of antibodies and lectins have been relied on for glycan detections. ${ }^{57}$ However, these methods come with a number of drawbacks. Although, antibodies with high specificity for distinct glycan epitopes exist, it is challenging to develop high-affinity antibodies for glycans in general. ${ }^{57}$ It is also difficult to develop antibodies that can distinguish between N-linked and O-linked glycans. ${ }^{57}$ Similarly, lectins typically have low affinities for certain glycan targets and often lack specificity. ${ }^{57}$ Instead, chemo-enzymatic labelling of glycans have shown to be more effective. This method utilizes glycosyltransferases for their inherent substrate specificity to transfer modified sugars onto target acceptor glycans. These modified sugars can be functionalized with a tag moiety for means of detection.

To date, sialic acids with a few modifications have been shown to be tolerated by sialyltransferases and therefore have been used as tags to detect glycosylation. ${ }^{54,68}$ However, only a few studies have been reported on the sialylation kinetics of sialyltransferases with exogenous glycoproteins and how they tolerate modified sialic acids. ${ }^{54}$ 


\subsubsection{Project objectives}

The overall goal of this project is to develop efficient BODIPY-sialic acid probes for enzyme studies. The Wakarchuk lab has been characterizing the enzymes that transfer sialic acids to various carbohydrate acceptors. Therefore, it is critical for the development of probes that allow easy enzymatic detection of specific glycoconjugates. Amongst these enzymes the sialyltransferases ST3Gal1 and ST6Gal1 will be investigated in this study. This project has been broken down into two aims:

1) Development and characterization of BODIPY-sialic acid probes.

2) Applying these BODIPY-sialic acid probes to study the ST3Gal1 and ST6Gal1. Since sialic acids with modifications at the C-5 and C-9 positions have been used as chemical tags to detect glycosylation, a BODIPY dye has been selected as a fluorescent label. Sialic aids used for this research will be functionalized with an azido group at the C-5 and C-9 position (Figure 28) allowing for the labelling with BODIPY-alkyne via CuAAc reaction. This study aims to determine the efficiency of human ST3Gal1 and ST6Gal1 enzymes to catalyze the transfer of C-5 and C-9 modified sialic acid donors onto different $\mathrm{O}$-linked and $\mathrm{N}$-linked glycan containing acceptor proteins, respectively. 


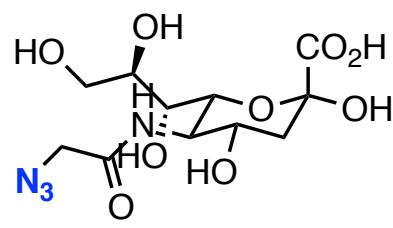

2.1 SiaNAz

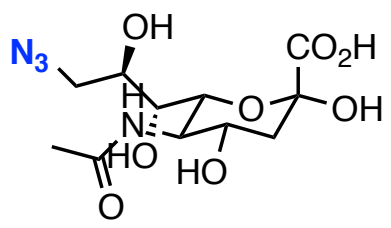

2.2 9-azido-Neu5Ac

Figure 28: Chemical structures of C-5 and C-9 azido modified sialic acids.

\subsubsection{Acceptor Proteins for Modification}

The following three asialoglycoproteins were used as acceptor proteins for the sialylation studies of ST3Gal1 and ST6Gal1. Two of the asialoglycoproteins used consist of N-linked glycans, while the third consists of an O-linked glycan.

a) Interferon alpha-2b (IFN $\alpha-2 b)$ : is a glycoengineered protein that contains a single core-1 (T antigen) 0-glycosylation site. This glycoengineered protein is a part of an ongoing project in the Wakarchuk lab, where we have been examining its synthesis. It would be interesting to see whether the BODIPYsialic acid probes can be used to detect the presence of Gal- $\beta 1,3-G a l N A c \alpha$.

b) Plant expressed human butyrylcholinesterase (BuChE): is another target protein part of another ongoing project in the Wakarchuk lab, where they have been looking at the in-vitro $\mathrm{N}$-glycan modifications of these plant derived BuChE in order to mimic human BuChE N-glycan chains.

c) Asialo-alpha 1 anti-trypsin (Asialo- $\alpha 1 \mathrm{AT}$ ): is a serine protein inhibitor comprising of N-glycosidically linked oligosaccharide chains. ${ }^{59}$ It is another target protein studied in the Wakarchuk lab, where in vitro protein 
modifications using sialyltransferases are being looked at. Therefore, easy ways to monitor and quantify the sialylation of $\mathrm{N}$-glycans are needed.

\subsection{Experimental}

\subsubsection{Materials}

9-aminoacridine, cytidine 5'-triphosphate (CTP), p-anisaldehyde was purchased from Sigma-Aldrich. Sialic acid aldolase was obtained from Carbosynth. Sodium pyruvate was obtained from Medstore University of Toronto (U of T). ManNAz compound was obtained from the Nitz Lab in the Department of Chemistry at the U of T. The 9-azido-Neu5Ac (2.2) was purchased from Sussex Research. 5 mL HiTrap Capto Q anion exchange column was purchased from GE Healthcare.

\subsubsection{Analysis of compounds by MALDI-TOF mass spectroscopy}

$1 \mu \mathrm{L}$ of a $15 \mathrm{mg} / \mathrm{mL}$ solution of 9-aminoacridine (9-AA) in isopropanol/acetonitrile (60:40) was transferred to the sample plate and dried. $1 \mu \mathrm{L}$ of the product solution (10 pmol/ $\mu \mathrm{L}$ made up in water) was then spotted on top of the matrix spot and dried. Spectra was acquired on a Autoflex Speed MALDI-TOF instrument employing a Nd:YAG laser (355 nm). Linear negative mode using accelerating voltage of $3047 \mathrm{~V}$ was used to obtain the spectra. Calibration performed using red phosphorous clusters. 


\subsubsection{Synthesis and Purification of BODIPY-Sialic acid Probes}

\subsubsection{Synthesis and Purification of SiaNAz}

A 30 mM ManNAz stock solution was prepared by dissolving ManNAz (10.4mg, $39.7 \mu \mathrm{mol})$ in a $50 \mathrm{mM}$ Tris $\mathrm{pH} 7.5$ buffer $(1.3 \mathrm{~mL})$. A $100 \mathrm{mM}$ sodium pyruvate stock solution was prepared by dissolving sodium pyruvate $(110 \mathrm{mg}, 1 \mathrm{mmol})$ in a $50 \mathrm{mM}$ Tris pH 7.5 buffer $(10 \mathrm{~mL})$. The siaNAz was then synthesized via an aldol condensation reaction according to a previously published procedure. ${ }^{79}$ In a 1.75 mL microcentrifuge tube, $333.3 \mu \mathrm{L}$ of $30 \mathrm{mM}$ ManNaz was combined with $500 \mu \mathrm{L}$ of $100 \mathrm{mM}$ sodium pyruvate, sialic acid aldolase $(3 \mathrm{mg}$ ) and $166.7 \mu \mathrm{L}$ of water. The 1 $\mathrm{mL}$ reaction mixture was incubated at room temperature for about 72 hours, rotating in the dark. Progress of reaction was monitored on an aluminum-backed TLC plate until no increased product formation was observed. $0.5 \mu \mathrm{L}$ of reaction was spotted on a TLC plate and was developed using a 4:2:1:0.1 mix of EtOAc:MeOH: $\mathrm{H}_{2} \mathrm{O}: \mathrm{HOAc}$ solvent. The developed TLC plate was then stained with a panisaldehyde stain and charred forming brown spots to indicate the presence of ManNAz $\left(\mathrm{R}_{\mathrm{f}} \approx 0.63\right)$ and siaNAz $(\mathrm{Rf} \approx 0.24)$ and a yellow spot to indicate the presence of sodium pyruvate $(\mathrm{Rf} \approx 0.46)$. The siaNAz was then purified using a mini macro-prep DEAE column. siaNAz was loaded onto the DEAE column and prewashed with water and eluted with $0.5 \mathrm{M}$ ammonium acetate (pH 7.5) buffer. The eluted fractions were then analyzed using an aluminum-backed TLC plate as described above to confirm target product was obtained. The siaNAz product was then further purified using a freeze drier to remove the ammonium acetate by re- 
suspending the sample in water and freezing drying subsequently. This step was repeated twice. From a $1 \mathrm{~mL}$ reaction $\sim 8 \mathrm{mg}$ of product was obtained. TLC-MS (ESI): $\left(\mathrm{C}_{11} \mathrm{H}_{18} \mathrm{~N}_{4} \mathrm{O}_{9}\right)[\mathrm{M}-\mathrm{H}]$; , calculated $m / z$ 349.10, found $m / z$ 349.1.

\subsubsection{Synthesis of activated azido-sialic acid-CMP substrates}

An enzymatic reaction was conducted using Neisseria meningitides CMP- $\beta$-sialic acid synthetase (NSY-05) to transfer cytidine 5'-monophosphate (CMP) onto the azidosialic acid substrates from the donor cytidine $5^{\prime}$-triphosphate (CTP). The assays were conducted in a final volume of $1 \mathrm{~mL}$, the following components were mixed in a $1.75 \mathrm{~mL}$ microcentrifuge tube: $5 \mathrm{mM} \mathrm{CTP}$ and $5 \mathrm{mM}$ azido-sialic acid substrate in $100 \mathrm{mM}$ Tris pH 8.5, $20 \mathrm{mM} \mathrm{MgCl}$, $100 \mathrm{mM}$ Tris pH 8.5, $0.2 \mathrm{mM}$ Dithiothreitol (DTT), $0.36 \mathrm{mg} / \mathrm{mL}$ NSY-05 and water to make up the final $1 \mathrm{~mL}$ volume. Reaction mixture was then incubated at $37^{\circ} \mathrm{C}$ for $\sim 3 \mathrm{~h}$, and progress of the reaction was monitored on an aluminum-backed TLC plate. $0.5 \mu \mathrm{L}$ of the reaction was spotted on a TLC plate and was developed using a 4:3:2:1 mix of EtOAc:MeOH: $\mathrm{H}_{2} \mathrm{O}: \mathrm{NH}_{4} \mathrm{OH}$. The developed TLC plate was then stained with p-anisaldehyde and charred.

\subsubsection{Purification of azido-sialic acid-CMP substrates}

Azido modified sialic acid-CMP substrates were purified on a $5 \mathrm{ml}$ HiTrap Capto Q anion exchange column attached to a ÄKTA pure chromatography system. $1 \mathrm{~mL}$ of reaction mixture was diluted with $10 \mathrm{mM}$ ammonium bicarbonate in a 1:5 (v/v). 
Diluted reaction mixture was loaded onto the column at $2 \mathrm{~mL} / \mathrm{min}$ flow rate and run with a $0-25 \%$ gradient flow of $0.5 \mathrm{M}$ ammonium bicarbonate buffer. The product was eluted out from the column at $\sim 20-25 \%$ of $0.5 \mathrm{M}$ ammonium bicarbonate while the excess CTP eluted from the column at $100 \%$ of $0.5 \mathrm{M}$ ammonium bicarbonate. The eluted fractions containing the azido modified sialic acid-CMP were pooled and immediately freeze dried. Dried down product were stored in $-80^{\circ} \mathrm{C}$ until further use.

\subsubsection{BODIPY-labelling of Neu5Ac-CMP substrates}

A $20 \mathrm{mM}$ azido modified sialic acid-CMP stock solutions were prepared by dissolving 9-azido-Neu5Ac-CMP (2.56 mg, $4 \mu \mathrm{mol})$ or siaNAz-CMP (2.62 mg, $4 \mu \mathrm{mol})$ in $200 \mu \mathrm{L}$ of $100 \mathrm{mM}$ Tris $\mathrm{pH}$ 8.5. The CuAAC reaction protocol outlined in section 2.5.1 was used. The progress of the reaction was monitored on a plastic-backed TLC plate until full conversion of product formation was observed. $0.5 \mu \mathrm{L}$ of the reaction was spotted on a TLC plate and was developed using a 4:2:1:0.1 mix of EtOAc:MeOH: $\mathrm{H}_{2} \mathrm{O}: \mathrm{HOAc}$ solvent. The developed TLC plate was then analyzed on the EZDOC system using a blue light tray for band quantification. The reaction mixture was then purified using either the preparative-TLC method outlined in section 2.4 or the superdex peptide 10/300 GL column outline in section 2.5.4. MALDI-TOF-MS: BODIPY-9-Neu5Ac-CMP $\left(\mathrm{C}_{37} \mathrm{H}_{48} \mathrm{BF}_{2} \mathrm{~N}_{10} \mathrm{O}_{16} \mathrm{P}\right)[\mathrm{M}-\mathrm{H}]^{-}$, calculated $\mathrm{m} / z$ 967.30, found $m / z$ 966.894. BODIPY-5-Neu5Ac-CMP $\left(\mathrm{C}_{37} \mathrm{H}_{48} \mathrm{BF}_{2} \mathrm{~N}_{10} \mathrm{O}_{17} \mathrm{P}\right)$ [M-H]; calculated $m / z$ 
983.29, found $m / z$ 983.273. Due to hydrolysis, ${ }^{1} \mathrm{H}$ NMR analysis could not be obtained.

\subsubsection{Human ST3Gal1 Modification of Interferon $\alpha-2 b$}

Reactions were performed in $40 \mu \mathrm{L}$ at $30^{\circ} \mathrm{C}$ and $4 \mu \mathrm{L}$ samples were taken at various time points. $1 \mathrm{mg} / \mathrm{mL}(32 \mu \mathrm{M})$ interferon alpha- $2 \mathrm{~b}(\mathrm{IFN} \alpha-2 \mathrm{~b})$ obtained from Wakarchuk lab) was modified using 50 mM HEPES pH 7.2, 0.05 mM BODIPYNeu5Ac-CMP donor, $0.1 \mathrm{mg} / \mathrm{mL}$ human ST3Gal1 (HUST-60, an MBP-fusion to ST3Gal 1). Reactions were stopped with 1\% SDS solution before running on 15\% SDS-PAGE. Samples at each time point were analyzed by SDS-PAGE and visualized by fluorescence (blue light tray) and Coomassie brilliant blue (CBB) staining on the EZDOC imaging system for band quantitation.

\subsubsection{Human ST6Gal1 Modification of Asialo-A1AT and BuChE}

Reactions were all performed in $40 \mu \mathrm{L}$ at $30^{\circ} \mathrm{C}$ and $4 \mu \mathrm{L}$ samples were taken at various time points. Asialo-alpha 1 anti-trypsin (asialo- $\alpha 1 \mathrm{AT}$ ) (obtained from the desialylation of commercial A1AT [prolastin c] from Grifols) and plant expressed human butyrylcholinesterase (BuChE) (obtained from PlantForm Corporation) were modified by incubating the proteins with human ST6Gal1 (HUST-119, MBP-fusion to ST6Gal1 obtained from Hirak Saxena in the Wakarchuk lab) using the following reaction conditions: $2 \mathrm{mg} / \mathrm{mL}$ asialo-A1AT (38 $\mu \mathrm{M})$ or BuChE $(30 \mu \mathrm{M}), 50 \mathrm{mM}$ HEPES pH 7.2, $10 \mathrm{mM} \mathrm{MgCl}_{2}, 0.01 \%$ Triton, $0.1 \mathrm{mM}$ BODIPY-Neu5Ac-CMP and 0.1 
$\mathrm{mg} / \mathrm{mL}$ HUST-119. Reactions were stopped with $1 \%$ SDS solution before running on 12\% SDS-PAGE. Samples at each time point were analyzed by SDS-PAGE and visualized by fluorescence (blue light tray) and Coomassie brilliant blue (CBB) staining on the EZDOC imaging system for band quantitation. 


\subsection{Results}

\subsubsection{Development of BODIPY-Neu5Ac probes}

\subsubsection{Synthesis of SiaNAz}

The siaNAz compound was successfully synthesized using the aldol condensation reaction from the starting materials ManNAz, sodium pyruvate and sialic acid aldolase (Figure 29A). It was evident that the addition of an excess amount of sodium pyruvate in the reaction increased the product yield. In this synthesis, a ManNAz to sodium pyruvate assay concentration ratio of 1:5 was used. In order to verify the production and the removal of the siaNAz material $(\mathrm{Rf} \approx 0.24)$, it was analyzed on a TLC plate along with the starting materials ManNAz $(\mathrm{Rf} \approx 0.63)$ and sodium pyruvate $(\mathrm{Rf} \approx 0.46)$ as shown below in Figure 29B. From TLC analyses no further product formation was observed past a $72 \mathrm{~h}$ time point. 
A)<smiles>CC(=O)C(=O)O[Na]</smiles>

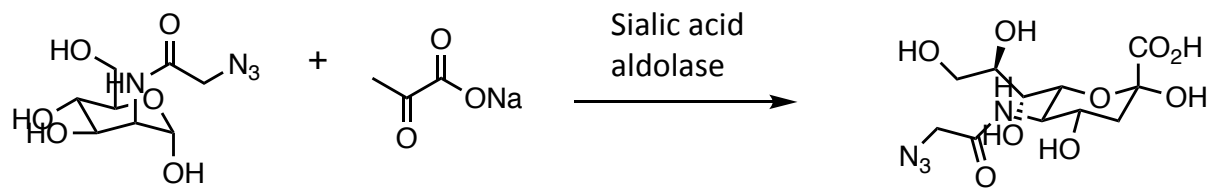
ManNAz sodium pyruvate

2.1 siaNAz

B)

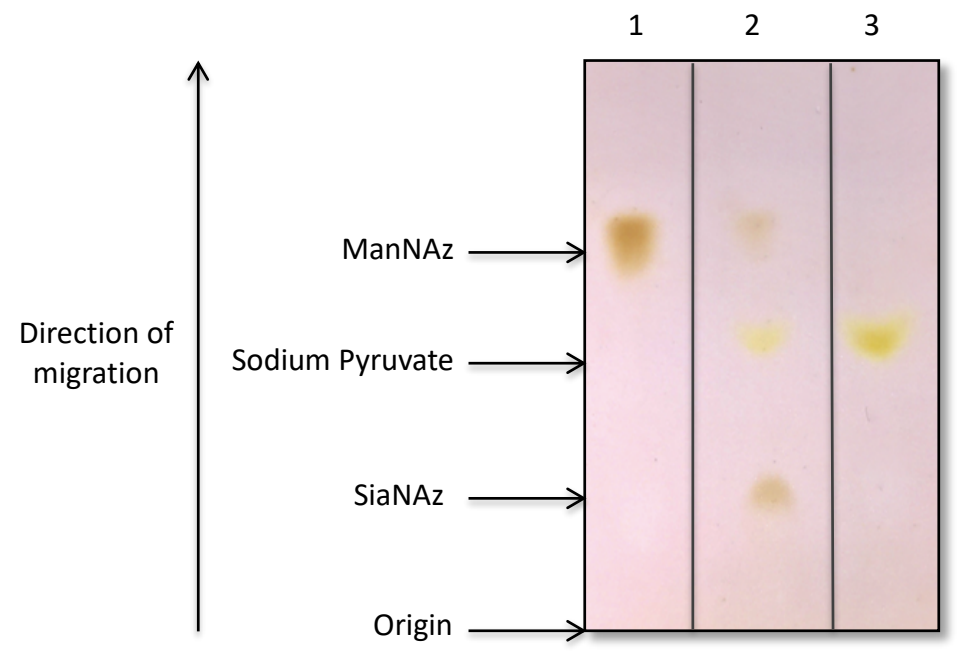

Figure 29: Synthesis of SiaNAz. A) Aldol condensation reaction scheme of siaNAz and B) TLC analysis of the siaNAz synthesis. Lane 1: starting material, ManNAz; Lanes 2: aldol condensation reaction, time point: 72h; Lane 3: starting material, sodium pyruvate. 


\subsubsection{Activation of azido-Neu5Ac-CMP substrates}

The activation of siaNAz- and 9-azido-Neu5Ac substrates were enzymatically synthesized by a bacterial Neisseria CMP- $\beta$-sialic acid synthetase (NSY-05) which transfers a cytidine 5'-triphosphate (CMP) onto the azido-Neu5Ac acceptor substrates from the donor cytidine $5^{\prime}$-triphosphate (CTP) in the presence of the metal ion, $\mathrm{Mg}^{2+}$ (Figure 30A). From the TLC results shown in Figure 30B, conversion to activated azido-Neu5Ac substrates were successfully achieved within $2 \mathrm{~h}$ of incubation. Due to the higher polarity of the CMP conjugated azido-Neu5Ac substrates it migrated much slower up the TLC plate in comparison to their inactivated counterparts. In addition, BODIPY-labelled Neu5Ac substrates can also be successfully activated under similar conditions within $3 \mathrm{~h}$ of incubation (Figure 31). 
A)

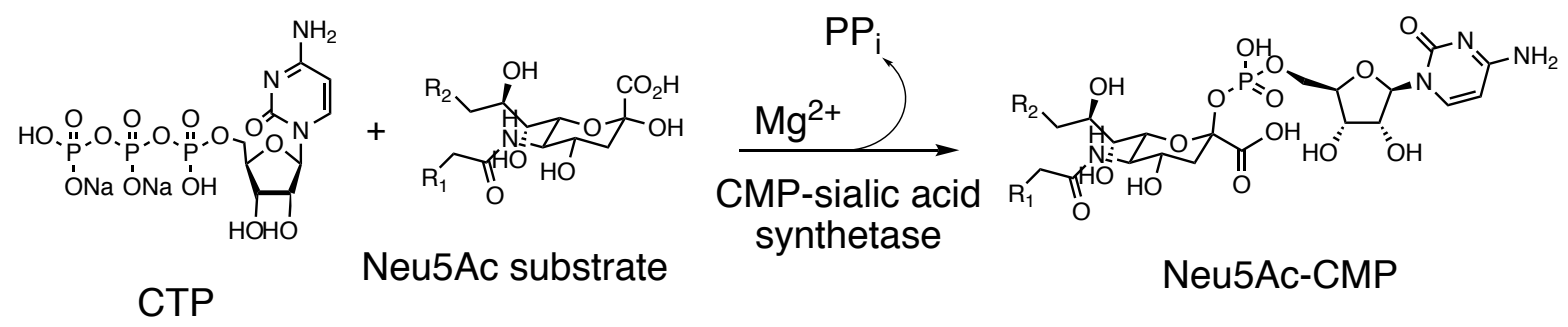

B)

I)

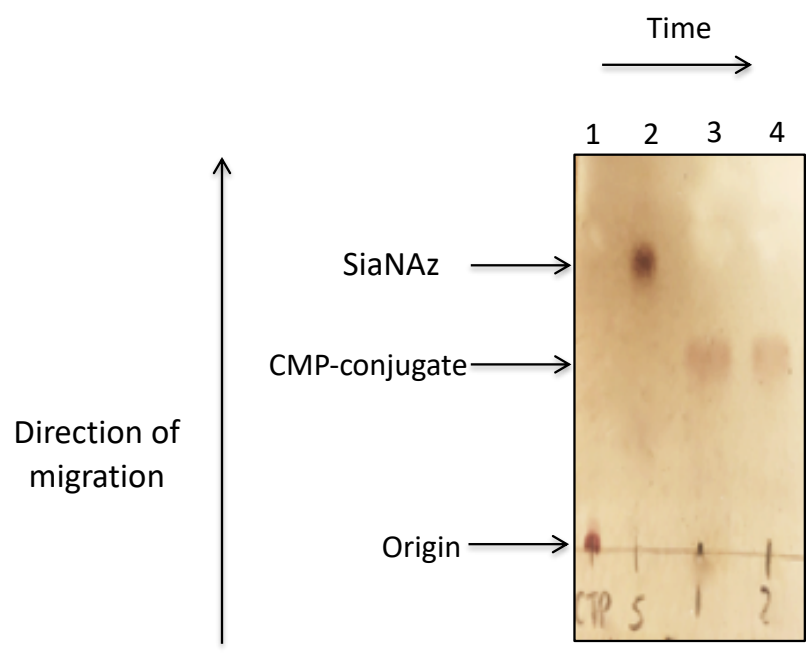

II)

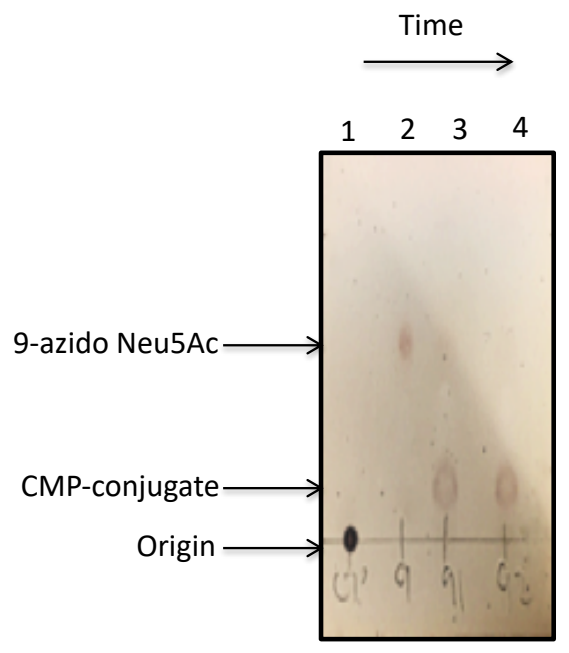

Figure 30: Activation of azido-Neu5Ac substrates via CMP- $\beta$-sialic acid synthetase (NSY-05) A) Reaction scheme of the azido-Neu5Ac-CMP substrates. C-5 modified Neu5Ac $\left(R_{1}=N_{3}, R_{2}=O H\right)$ and C-9 modified Neu5Ac $\left(R_{1}=H, R_{2}=N_{3}\right]$. B) TLC analysis of CMP-conjugate synthesis. I) Synthesis of siaNAz-CMP and; II) Synthesis of 9-azidoNeu5Ac-CMP. Lane 1: starting material, CTP; Lane 2: Negative control, NSY-05 absent; Lanes 3-4, NSY-05 present, time points: $2 \mathrm{~h}$ and $3 \mathrm{~h}$. 
A)

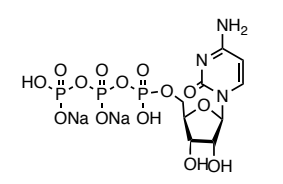

CTP

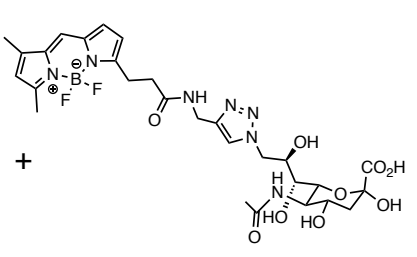

BODIPY-9-Neu5Ac

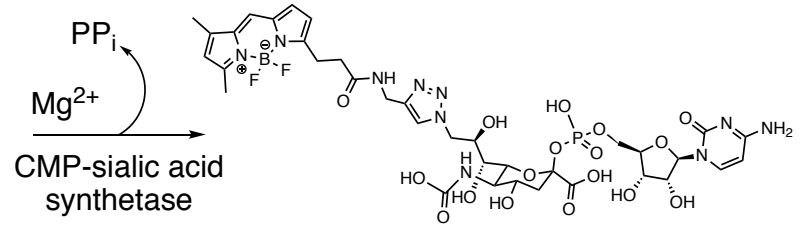

2.3 BODIPY-9-Neu5Ac-CMP

B)

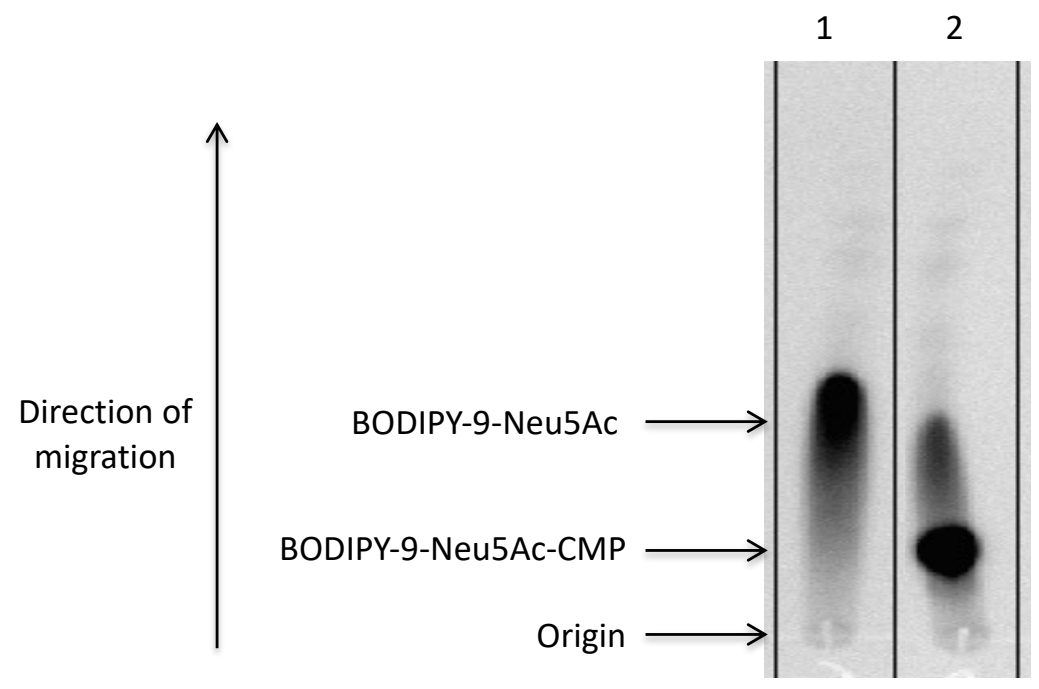

Figure 31: Activation of BODIPY-9-Neu5Ac via CMP- $\beta$-sialic acid synthetase (NSY-05). A) Reaction scheme of the BODIPY-9-Neu5Ac-CMP substrates. B) TLC analysis of BODIPY-9-Neu5Ac-CMP synthesis. Lane 1: Negative control, NSY-05 absent. Lanes 2: NSY-05 present, time point: $3 \mathrm{~h}$.

\subsubsection{BODIPY-labelled Neu5Ac-CMP derivatives}

Labelling of activated-Neu5Ac-CMP derivatives was done using the CuAAc reaction outlined in Figure 5. In this reaction the BODIPY-alkyne (I) was 'clicked' onto the azido-Neu5Ac-CMP substrates generating a stable covalently linked BODIPYlabelled Neu5Ac-CMP substrates. Within $1 \mathrm{~h}$ of incubating about 90 and $95 \%$ 
relative conversion to BODIPY-5-Neu5Ac-CMP and BODIPY 9-Neu5Ac-CMP occurred, respectively (Figure 32). The labelling can also successfully be done prior to the activation of azido sialic acid (data not shown).

A)

I)

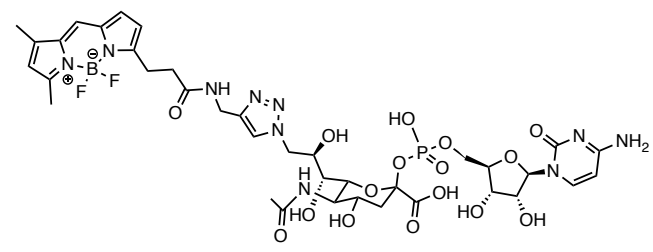

2.3 BODIPY-9-Neu5Ac-CMP

II)

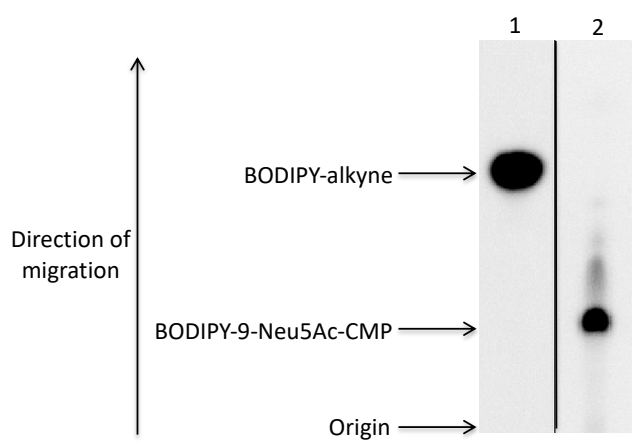

B)

I)

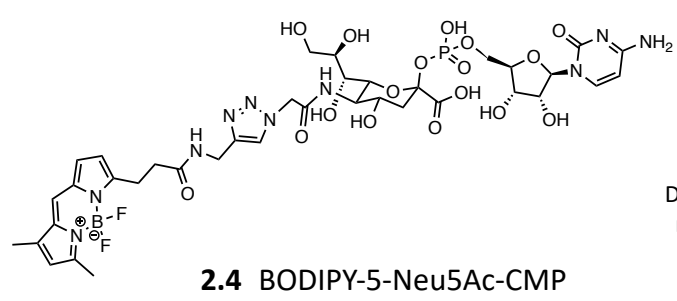

II)

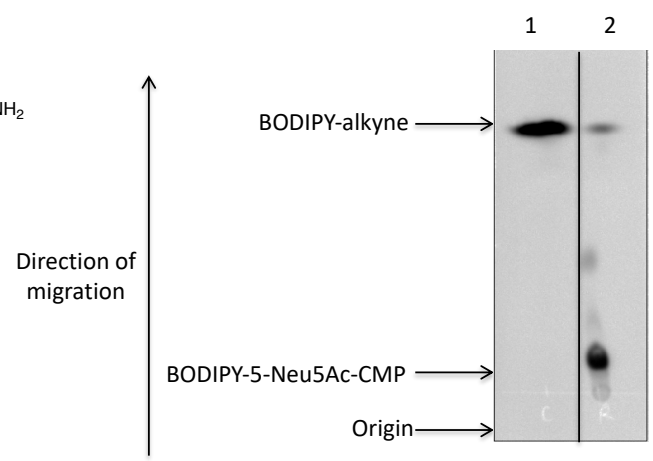

Figure 32: Synthesis of BODIPY-labelled Neu5Ac-CMP derivatives using CuAAc reaction. A) BODIPY-9-Neu5Ac-CMP: I) chemical structure and; II) TLC analysis of BODIPY-9-Neu5Ac-CMP synthesis, Lane 1: starting material, BODIPY-alkyne; and Lane 2: CuAAc reaction mixture, time point 1hr. B) BODIPY-5-Neu5Ac-CMP: I) chemical structure and; II) TLC analysis of BODIPY-5-Neu5Ac-CMP synthesis, Lane 1: starting material, BODIPY-alkyne; and Lane 2: CuAAc reaction, time point $1 \mathrm{hr}$. 


\subsubsection{Functional testing of BODIPY-Neu5Ac probes on proteins}

\subsubsection{Sialylation of IFN $\alpha$-2b using Human ST3Gal1}

The BODIPY-Neu5Ac-CMP probes were used to investigate the human ST3Gal1 and to compare its efficiency to transfer both probes onto $1 \mathrm{mg} / \mathrm{mL}$ of the protein acceptor interferon alpha-2b (IFN $\alpha-2 b)$, which contained a single T antigen modification. From the SDS-PAGE gels and the densitometric data obtained, was able to successfully transfer both the C-9 and C-5 modified Neu5Ac substrates onto IFN $\alpha$-2b (Figure 33). Within 15 minutes of incubation, transfer of both the BODIPY9- and BODIPY-5-Neu5Ac-CMP were observed, which increased with time, clearly illustrated in the fluorescently imaged gels. However, BODIPY-9-Neu5Ac-CMP substrate was seen to be transferred $\sim 7$ folds more than the BODIPY-5-Neu5AcCMP substrate, which was evident from the thicker and brighter protein bands seen on the fluorescence image. 
A)

I)

II)
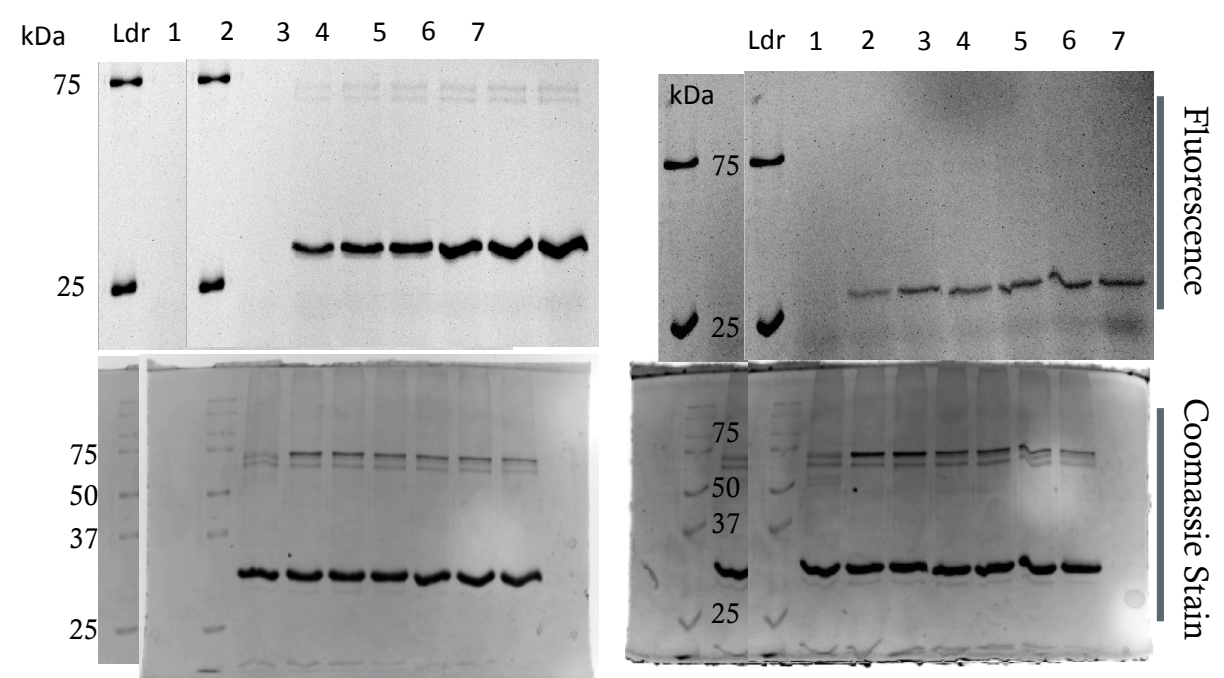

B)

\begin{tabular}{|c|c|c|c|c|c|c|c|}
\hline \multicolumn{2}{|c|}{ Probe: } & \multicolumn{3}{c|}{ BODIPY-9-Neu5Ac-CMP } & \multicolumn{3}{c|}{ BODIPY-5-Neu5Ac-CMP } \\
\hline & $\begin{array}{c}\text { Time } \\
\text { Lane }\end{array}$ & CBB & Fluorescence & Ratio & CBB & Fluorescence & Ratio \\
\hline 1 & - & - & - & - & - & - & - \\
\hline 2 & 15 & 1569414 & 473040 & 0.3 & 1326340 & 8064 & 0.04 \\
\hline 3 & 30 & 1533425 & 666525 & 0.43 & 1365082 & 100926 & 0.07 \\
\hline 4 & 60 & 1587357 & 848610 & 0.53 & 1392895 & 115182 & 0.08 \\
\hline 5 & 120 & 1678540 & 1114200 & 0.66 & 1542475 & 136323 & 0.08 \\
\hline 6 & 180 & 1872200 & 1264112 & 0.67 & 1540200 & 138432 & 0.09 \\
\hline 7 & $0 / N$ & 1912608 & 1560800 & 0.81 & 1430190 & 128775 & 0.09 \\
\hline
\end{tabular}

Figure 33: Comparison of BODIPY-Neu5Ac-CMP probes in the modification of IFN $\alpha-2 b$ using human ST3Gal1. A) SDS-PAGE comparison of the probes (0.05mM): I) BODIPY-9Neu5Ac-CMP and ii) BODIPY-5-Neu5Ac-CMP. B) Densitometric analysis: a quantitative study to determine which probe is efficiently transferred onto $1 \mathrm{mg} / \mathrm{mL}$ protein using $0.1 \mathrm{mg} / \mathrm{mL}$ ST3Gal1. Coomassie blue staining (CBB). Lane 1: Negative control;

ST3Gal1 absent. Lanes 2-7: ST3Gal1 present, time points 15, 30, 60, 120, 180 min and overnight. 


\subsubsection{Sialylation of BuChe and AS- $\alpha 1 A T$ Human ST6Gal1}

The BODIPY-Neu5Ac-CMP probes were used to study the human ST6Gal1 and to compare its efficiency to transfer both probes onto two different N-glycan containing protein acceptors; asialo-alpha 1 anti-trypsin (asialo- $\alpha 1 \mathrm{AT}$ ) and plant expressed human butyrylcholinesterase (BuChE). Human ST6Gal1 was investigated for its ability to modify $2 \mathrm{mg} / \mathrm{mL}$ of target proteins.

For the analysis on BuChE, the SDS-PAGE gels and the densitometric data obtained showed that ST6Gal1 was able to successfully transfer both the BODIPY-5and BODIPY-9-Neu5Ac-CMP substrates at about the same rate (Figure 34). Within 15 minutes of incubation, transfer of both the BODIPY-9- and BODIPY-5-Neu5AcCMP were observed, which increased with time, illustrated in the fluorescently imaged gels.

Whereas for asialo-A1AT, the results obtained showed that ST6Gal1 transfers both the BODIPY-5- and BODIPY-9-Neu5Ac-CMP substrates at completely different rates (Figure 35). The ST6Gal1 was observed to transfer BODIPY-5-Neu5Ac-CMP much more efficiently compared to BODIPY-9-Neu5Ac-CMP, where only faint bands can be detected on the fluorescently imaged gel. 
A)

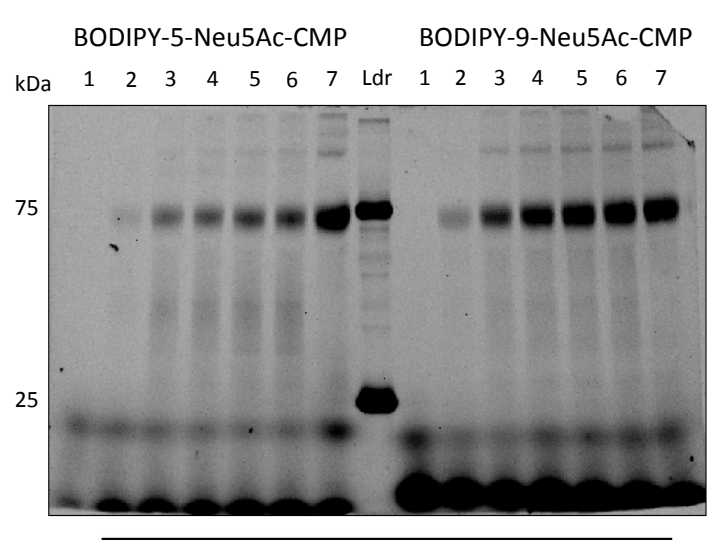

Fluorescence
BODIPY-5-Neu5Ac-CMP BODIPY-9-Neu5Ac-CMP

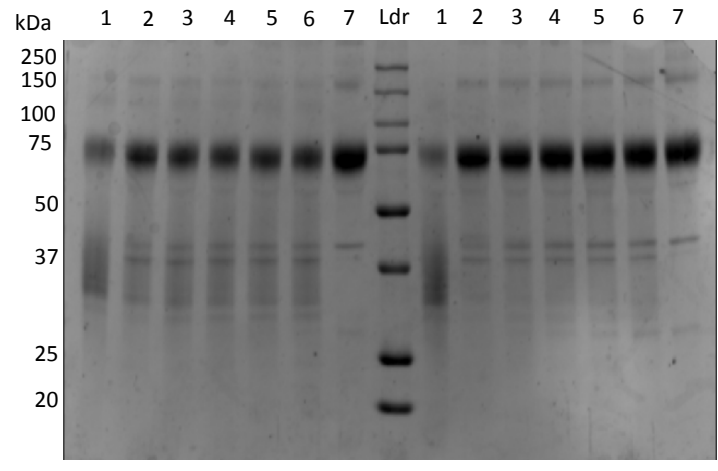

Coomassie Staining

B)

\begin{tabular}{|c|c|c|c|c|c|c|c|}
\hline \multicolumn{2}{|c|}{ Probe: } & \multicolumn{3}{c|}{ BODIPY-5-Neu5Ac-CMP } & \multicolumn{3}{c|}{ BODIPY-9-Neu5Ac-CMP } \\
\hline Lane & $\begin{array}{c}\text { Time } \\
\text { (min) }\end{array}$ & CBB & Fluorescence & Ratio & CBB & Fluorescence & Ratio \\
\hline 1 & - & - & - & - & - & - & - \\
\hline 2 & 0 & 1256066 & 41940 & 0.03 & 1499841 & 94410 & 0.06 \\
\hline 3 & 30 & 1060262 & 146025 & 0.14 & 1390914 & 276570 & 0.20 \\
\hline 4 & 60 & 927472 & 175860 & 0.19 & 1606955 & 393000 & 0.24 \\
\hline 5 & 120 & 926262 & 223920 & 0.24 & 1660120 & 477450 & 0.29 \\
\hline 6 & 240 & 901600 & 244485 & 0.27 & 1679279 & 494595 & 0.29 \\
\hline 7 & $0 / N$ & 1687560 & 615105 & 0.36 & 1955835 & 560612 & 0.29 \\
\hline
\end{tabular}

Figure 34: Comparison of BODIPY-Neu5Ac-CMP probes in the modification of BuChE using human ST6Gal1. A) SDS-PAGE comparison of the probes (0.1 mM) BODIPY-9Neu5Ac-CMP and BODIPY-5-Neu5Ac-CMP. B) Densitometric analysis: a quantitative study to determine which probe is efficiently transferred onto $2 \mathrm{mg} / \mathrm{mL}$ protein using $0.1 \mathrm{mg} / \mathrm{mL}$ ST6Gal1. Coomassie blue staining (CBB). Lane 1: Negative control; ST6Gal1 absent. Lanes 2-7: ST6Gal1 present, time points 0, 30, 60, 120, 240 min and overnight. 
A)

BODIPY-9-Neu5Ac-CMP BODIPY-5-Neu5Ac-CMP

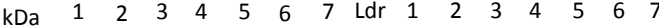

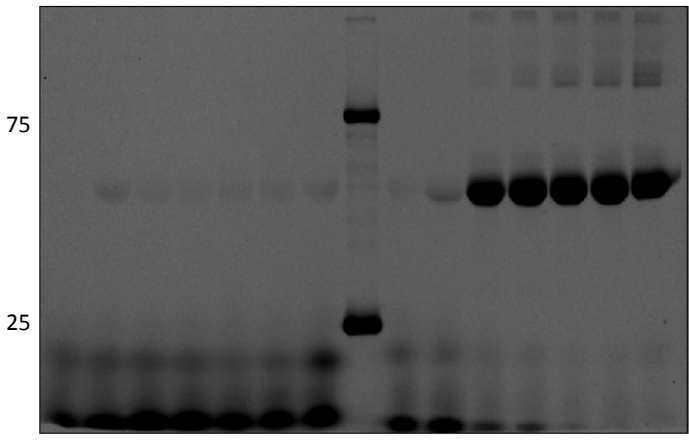

Fluorescence

BODIPY-9-Neu5Ac-CMP BODIPY-5-Neu5Ac-CMP

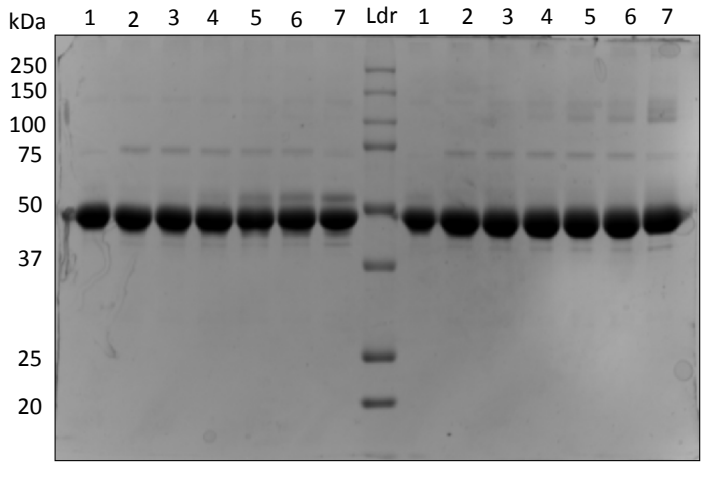

Coomassie Staining

B)

\begin{tabular}{|c|c|c|c|c|c|c|c|}
\hline \multicolumn{2}{|c|}{ Probe: } & \multicolumn{3}{c|}{ BODIPY-9-Neu5Ac-CMP } & \multicolumn{3}{c|}{ BODIPY-5-Neu5Ac-CMP } \\
\hline Lane & $\begin{array}{c}\text { Time } \\
\text { (min) }\end{array}$ & CBB & Fluorescence & Ratio & CBB & Fluorescence & Ratio \\
\hline 1 & - & - & - & - & - & - & - \\
\hline 2 & 0 & 3799368 & 13024 & 0.00 & 3636620 & 245646 & 0.07 \\
\hline 3 & 30 & 3111601 & 12362.5 & 0.00 & 3443371 & 1617162 & 0.47 \\
\hline 4 & 60 & 3328523 & 12128 & 0.00 & 3098535 & 1718182 & 0.55 \\
\hline 5 & 120 & 3065923 & 10331.5 & 0.00 & 3395575 & 1940178 & 0.57 \\
\hline 6 & 240 & 3342744 & 21544 & 0.01 & 3570709 & 2046551 & 0.57 \\
\hline 7 & $0 / N$ & 4523463 & 21019 & 0.00 & 3988411 & 2469144 & 0.62 \\
\hline
\end{tabular}

Figure 35: Comparison of BODIPY-Neu5Ac-CMP probes in the modification of AS$\alpha 1 A T$ using human ST6Gal1. A) SDS-PAGE comparison of the probes (0.1mM) BODIPY9-Neu5Ac-CMP and BODIPY-5-Neu5Ac-CMP. B) Densitometric analysis: a quantitative study to determine which probe is efficiently transferred onto $2 \mathrm{mg} / \mathrm{mL}$ protein using $0.1 \mathrm{mg} / \mathrm{mL}$ ST6Gal1. Coomassie blue staining (CBB). Lane 1: Negative control; ST6Gal1 absent. Lanes 2-7: ST6Gal1 present, time points 0, 30, 60, 120, 240 min and overnight. 


\subsection{Discussion}

To evaluate the transfer efficiencies of the human sialyltransferases, two sialic acid probes with modifications on the C-5 and C-9 positions were successfully developed. The siaNAz substrate was initially synthesized via an aldol condensation reaction and successfully purified by anion-exchange chromatography, which was later confirmed by ESI-MS analysis. Activated sialic acid derivatives were then enzymatically synthesized by a bacterial Neisseria CMP- $\beta$-sialic acid synthetase (NSY-05). The activation can occur before or after the labelling with BODIPY, as both routes generated relatively high yields of $90 \%$ or greater. An issue to take into consideration was that activated sialic acids (CMP-sialic acid) are in fact prone to chemical hydrolysis especially if kept in solution for too long, eventually leading to a 1:1 mixture of free CMP and deactivated sialic acids ${ }^{61}$. A reason for this is due to the additional electron-withdrawing effect of their anomeric carboxylate group ${ }^{61}$. To prevent this issue, activated sialic acids were freeze dried and the unused fractions were stored at $-80^{\circ} \mathrm{C}$ until further use. Subsequently, the azido modifications at the C-5 and C-9 positions allowed for the quick and efficient labelling with the BODIPYalkyne (I) through the CuAAc reaction, which was later confirmed by MALDI-TOFMS. Each of the BODIPY-5 and BODIPY-9-Neu5Ac-CMP substrates were directly used with both the ST3Gal1 and ST6Gal1.

An in vitro sialyltransferase assay was developed based on asialoglycoproteins as acceptor substrates for testing the efficiencies of human ST3Gal1 and ST6Gal1 for transferring the donor substrates BODIPY-5 and BODIPY- 
9-Neu5Ac-CMP. The ST3Gal1 studies were performed on 0-linked glycoproteins, whereas ST6Gal1 studies were performed on N-linked glycoproteins. From the results described above, ST3Gal1 efficiently recognized and sialylated the terminal Gal residue on the single $\mathrm{T}$ antigen found in interferon alpha-2b (IFN $\alpha-2 b)$. Both the sialic acid donor substrates were seen to be transferred by ST3Gal1, however BODIPY-9-Neu5Ac-CMP substrate was transferred $\sim 7$ folds more than the BODIPY5-Neu5Ac-CMP substrate. Suggesting that there is better recognition of the C-9 modified sialic acid by the IFN $\alpha$-2b glycoprotein.

For ST6Gal1 studies, different transfer profiles were obtained for the two Nglycan containing acceptor proteins, butyrylcholinesterase (BuChE) and asialoalpha 1 anti-trypsin (asialo- $\alpha 1 \mathrm{AT}$ ). From the results described above, ST6Gal1 was observed to effectively modify BuChE with both the C-5 and C-9 modified sialic acid donors. Both donor substrates were seen to be transferred onto BuChE at a relatively similar rate, suggesting that this glycoprotein does not discriminate between the two modifications. Whereas for asialo-A1AT, ST6Gal1 was only able to efficiently transfer the BODIPY-5-Neu5Ac-CMP substrate. Really low transfer was observed with the BODIPY-9-Neu5Ac-CMP substrate visualized by SDS-PAGE, signifying that there is better recognition of the C-5 modified sialic acid by the asialo-A1AT glycoprotein. 


\subsection{Concluding Remarks}

Given that sialic acids are typically found on the terminal ends of glycoconjugates, it is not surprising that they are essential in several biological roles. Therefore, developing simple methods for probing sialylation is an important research interest, as it allows for the manipulation and control of sialylation levels, as well as elucidating the wide-ranging biological functions of sialic acids. This chapter involved the successful development of BODIPY-sialic acid probes with azido modifications on the C-5 and C-9 positions of sialic acid, which allowed for the quick and efficient labelling with BODIPY dyes. It was found that these BODIPY-sialic acid derivatives can be transferred onto asialoglycoproteins with human ST3Gal1 and ST6Gal1 sialyltransferases. Differences in transfer efficiencies were observed indicating the specificity of the sialyltransferases and asialoglycoproteins for modifications of sialic acids at either the C-5 or C-9 positions. This study outlined the application of these BODIPY-sialic acid probes as a rapid analytical tool for the detection of T-antigen (Gal- $\beta 1,3$-GalNAc $\alpha$ ) 0-glycans on glycoproteins. As well as using them as an assay for monitoring in vitro protein sialylation on $\mathrm{N}$-glycans.

\subsubsection{Future Work}

Future studies with these BODIPY-sialic acid probes could involve surveying the transfer efficiencies of other mono-sialyltransferases and polysialyltransferases not just from mammalian cell lines, but also bacterial derivatives as well. In addition, using these as rapid analytical tools in proteomics studies could also be examined 
owing to their specificity and selectivity. For instance, the deficiencies of sialic acids in endothelial cells can be investigated, by using these probes to identify and isolate the subset of glycoproteins that are missing the sialic acid residues that specific sialyltransferases add. 


\section{Appendices}

List of Appendix Figures

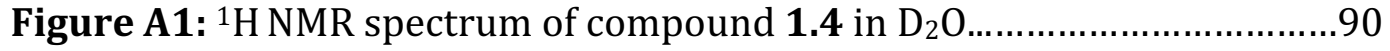

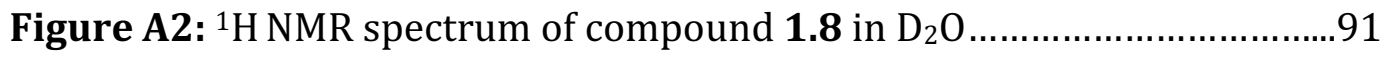

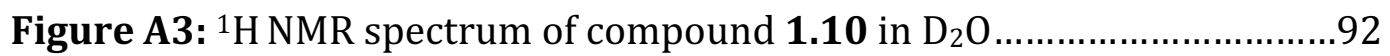




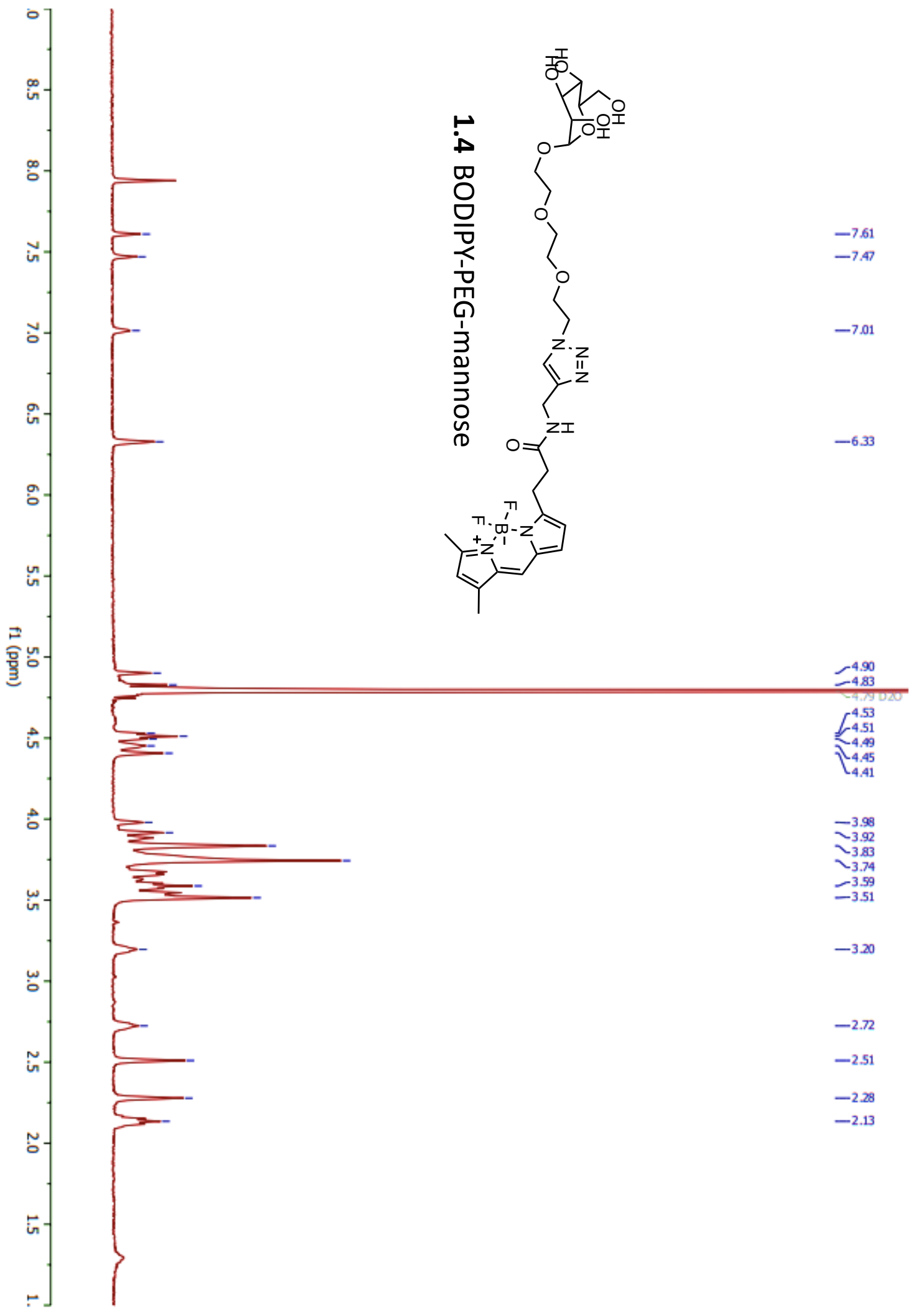

Figure A1: ${ }^{1} \mathrm{H}$ NMR spectrum of compound 1.4 in $\mathrm{D}_{2} \mathrm{O}$ 


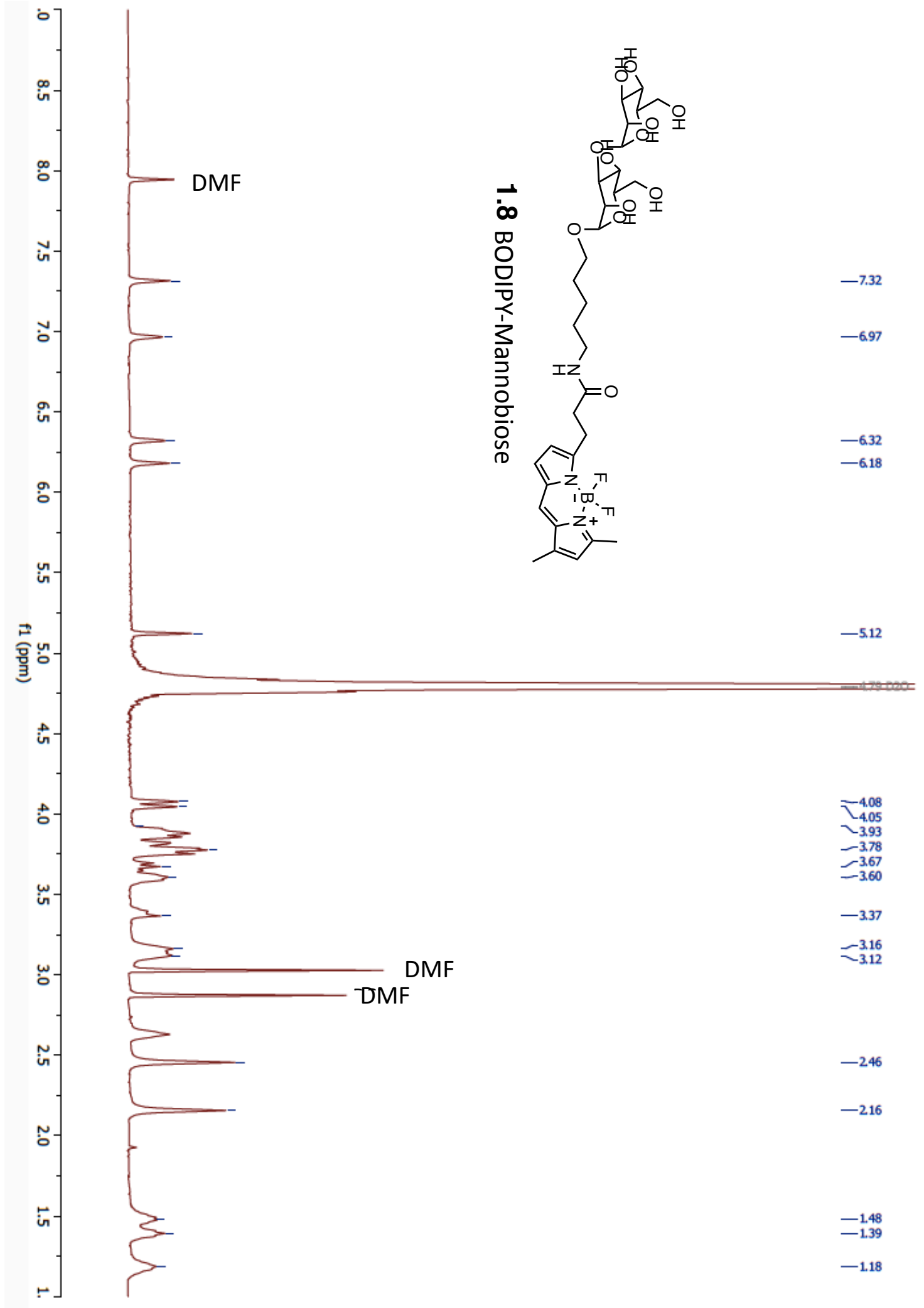

Figure A2: ${ }^{1} \mathrm{H}$ NMR spectrum of compound 1.8 in $\mathrm{D}_{2} \mathrm{O}$ 


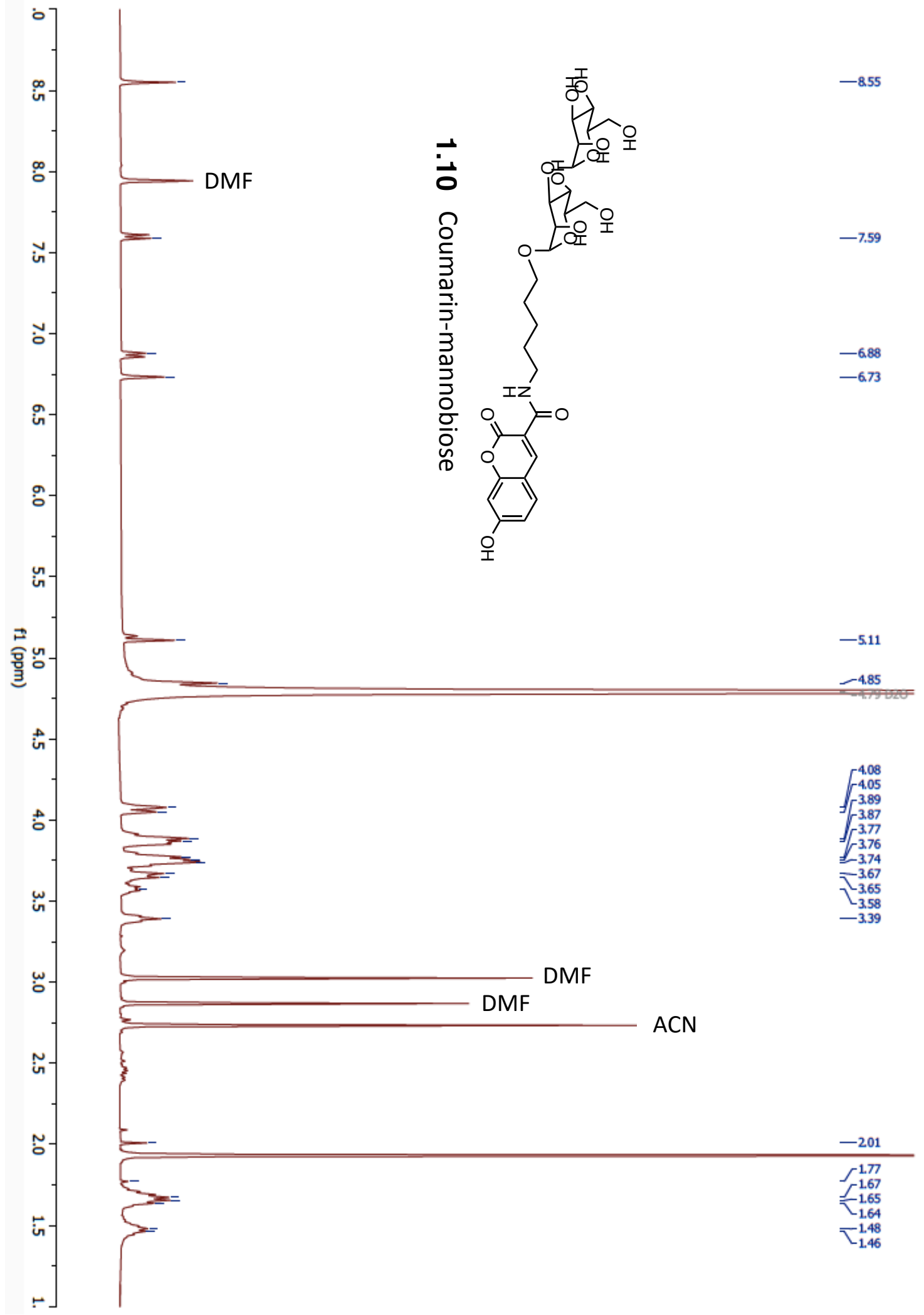

Figure A3: ${ }^{1} \mathrm{H}$ NMR spectrum of compound 1.10 in $\mathrm{D}_{2} \mathrm{O}$ 


\section{References}

1. François, K. O., \& Balzarini, J. (2012). Potential of carbohydrate-binding agents as therapeutics against enveloped viruses. Medicinal Research Reviews, 32(2), 349-387.

2. Varki A, Cummings RD, Esko JD, et al., editors. Essentials of Glycobiology. 3rd edition. Cold Spring Harbor (NY): Cold Spring Harbor Laboratory Press; 20152017. Available from: https://www.ncbi.nlm.nih.gov/books/NBK310274/

3. Sansone, F., \& Casnati, A. (2013). Multivalent glycocalixarenes for recognition of biological macromolecules: Glycocalyx mimics capable of multitasking. Chemical Society Reviews, 42(11), 4623-4639.

4. Gupta, G.S. (2012). Animal Lectins: Form, Function and Clinical Applications. Springer Vienna.

5. Jayawardena, H. S. N., Wang, X., \& Yan, M. (2013). Classification of lectins by pattern recognition using glyconanoparticles. Analytical Chemistry, 85(21), 10277.

6. Zhang, X., \& Yadavalli, V. K. (2009). Functionalized self-assembled monolayers for measuring single molecule lectin carbohydrate interactions. Analytica Chimica Acta, 649(1), 1-7.

7. Jayapalan, J. J., Lee, C., \& Hashim, O. H. (2017). Lectins: An effective tool for screening of potential cancer biomarkers. Peerj, 5, e3784.

8. Kim, D. H., Patnaik, B. B., Seo, G. W., Kang, S. M., Lee, Y. S., Lee, B. L., \& Han, Y. S. (2013). Identification and expression analysis of a novel R-type lectin from the coleopteran beetle, tenebrio molitor. Journal of Invertebrate Pathology, 114(3), 226-229.

9. Liu, Z., Li, X., Ding, X., \& Yang, Y. (2010). In silico and experimental studies of concanavalin A: Insights into its antiproliferative activity and apoptotic mechanism. Applied Biochemistry and Biotechnology, 162(1), 134.

10. Asthana, S., Bhutia, S. K., Sahoo, H., \& Jha, S. (2017). Chaotropes trigger conformational rearrangements differently in concanavalin A. Journal of Chemical Sciences, 129(8), 1267-1276.

11. Kussrow, A., Kaltgrad, E., Wolfenden, M. L., Cloninger, M. J., Finn, M. G., \& Bornhop, D. J. (2009). Measurement of monovalent and polyvalent carbohydrate-lectin binding by back-scattering interferometry. Analytical Chemistry, 81(12), 4889-4897. 
12. Shang, K., Song, S., Cheng, Y., Guo, L., Pei, Y., Lv, X., Pei, Z. (2018). Fabrication of carbohydrate chips based on polydopamine for real-time determination of Carbohydrate ${ }^{-}$Lectin interactions by QCM biosensor. Polymers, 10(11), 1275.

13. Rüdiger, H., \& Gabius, H. (2001). Plant lectins: Occurrence, biochemistry, functions and applications. Glycoconjugate Journal, 18(8), 589-613.

14. Alessandra, C., Marjolein, K., \& G, F. C. (2005). How C-type lectins detect pathogens. Cellular Microbiology, 7(4), 481-488.

15. De Messias-Reason, I., \& Boldt, A. B. W. (2009). Mannose-binding lectin in the innate immune system Nova Science.

16. Galanina, O., Feofanov, A., Tuzikov, A. B., Rapoport, E., Crocker, P. R., Grichine, A., Bovin, N. V. (2001). Fluorescent carbohydrate probes for cell lectins. Spectrochimica Acta Part A: Molecular and Biomolecular Spectroscopy, 57(11), 2285-2296.

17. Müller, C., Despras, G., \& Lindhorst, T. K. (2016). Organizing multivalency in carbohydrate recognition. Chemical Society Reviews, 45(11), 3275-332.

18. Lee, M., Park, S., \& Shin, I. (2006). Protein microarrays to study carbohydraterecognition events. Bioorganic \& Medicinal Chemistry Letters, 16(19), 51325135.

19. Disney, M. D., Zheng, J., Swager, T. M., \& Seeberger, P. H. (2004). Detection of bacteria with carbohydrate-functionalized fluorescent polymers. Journal of the American Chemical Society, 126(41), 13343-13346.

20. Wessig, P., Behrends, N., Kumke, M. U., Eisold, U., Meiling, T., \& Hille, C. (2016). Two-photon FRET pairs based on coumarin and DBD dyes. RSC Adv, 6(40), 33510-33513.

21. Fei, X., \& Gu, Y. (2009). Progress in modifications and applications of fluorescent dye probe. Progress in Natural Science, 19(1), 1-7.

22. Ulrich, G., Ziessel, R., \& Harriman, A. (2008). The chemistry of fluorescent bodipy dyes: Versatility unsurpassed. Angewandte Chemie (International Ed. in English), 47(7), 1184-1201.

23. Schmitt, A., Hinkeldey, B., Wild, M., \& Jung, G. (2009). Synthesis of the core compound of the BODIPY dye class: 4,4'-difluoro-4-bora-(3a,4a)-diaza-sindacene. Journal of Fluorescence, 19(4), 755-758. 
24. M. Ravikanth, M., Vellanki, L., \& Sharma, R. (2016). Functionalized borondipyrromethenes and their applications. Reports in Organic Chemistry, 6, 1.

25. Loudet, A., \& Burgess, K. (2007). BODIPY dyes and their derivatives: Syntheses and spectroscopic properties. Chemical Reviews, 107(11), 48914932.

26. Garland, M., Yim, J. J., \& Bogyo, M. (2016). A bright future for precision medicine: Advances in fluorescent chemical probe design and their clinical application. Cell Chemical Biology, 23(1), 122-136.

27. Mansour, F. R., Zhou, L., \& Danielson, N. D. (2015). Applications of poly(ethylene)glycol (PEG) in separation science. Chromatographia, 78(23), 1427-1442.

28. Bader, D., Klier, D. T., Hettrich, C., Bier, F. F., \& Wessig, P. (2016). Detecting carbohydrate-lectin interactions using a fluorescent probe based on DBD dyes. Anal. Methods, 8(6), 1235-1238.

29. Banerjee, S. S., Aher, N., Patil, R., \& Khandare, J. (2012). Poly (ethylene glycol)Prodrug Conjugates: Concept, Design, and Applications. Journal of Drug Delivery, 2012, 103973.

30. Lauc, G., Lee, R. T., Dumiae, J., \& Lee, Y. C. (2000). Photoaffinity glycoprobes-a new tool for the identification of lectins. Glycobiology, 10(4), 357-364.

31. Sauer, M. M., Jakob, R. P., Eras, J., Baday, S., Eriș, D. (2016). Catch-bond mechanism of the bacterial adhesin FimH. Nature Communications, 7, 10738.

32. Bojarová, P., \& Křen, V. (2016). Sugared biomaterial binding lectins: Achievements and perspectives. Biomaterials Science, 4(8), 1142-1160.

33. Biotium (n.d.) Tech Tip: Imaging Bacteria Using Agarose Pads. Retrieved from https://biotium.com/tech-tips/tech-tip-imaging-bacteria-using-agarosepads/

34. Schnaar, R. L. (2015). Glycans and glycan-binding proteins in immune regulation: A concise introduction to glycobiology for the allergist. The Journal of Allergy and Clinical Immunology, 135(3), 609-615.

35. Yu, H., Yu, H., Karpel, R., \& Chen, X. (2004). Chemoenzymatic synthesis of CMP-sialic acid derivatives by a one-pot two-enzyme system: Comparison of substrate flexibility of three microbial CMP-sialic acid synthetases. Bioorganic \& Medicinal Chemistry, 12(24), 6427-6435. 
36. Angata, T., \& Varki, A. (2002). Chemical diversity in the sialic acids and related $\alpha$-keto acids: An evolutionary perspective. Chemical Reviews, 102(2), 439470

37. Haines-Menges, B. L., Whitaker, W. B., Lubin, J. B., \& Boyd, E. F. (2015). Host sialic acids: A delicacy for the pathogen with discerning taste. Microbiology Spectrum, 3(4)

38. Dhar, C., Sasmal, A., \& Varki, A. (2019). From "Serum sickness" to "Xenosialitis": Past, present, and future significance of the non-human sialic acid Neu5Gc. Frontiers in Immunology, 10

39. Ji, S., Wang, F., Chen, Y., Yang, C., Zhang, P., Zhang, X., Wang, B. (2017). Developmental changes in the level of free and conjugated sialic acids, Neu5Ac, Neu5Gc and KDN in different organs of pig: A LC-MS/MS quantitative analyses. Glycoconjugate Journal, 34(1), 21-30.

40. Varki, A., Cummings, R. D., Esko, J. D., Freeze, H. H., Stanley, P., Bertozzi, C. R., Hart, G. W., Etzler, M. E. (2009). Essentials of Glycobiology. Cold Spring Harbor Laboratory Press.

41. Zhang, Q., Wang, Y., Zheng, Q., \& Li, J. (2019). Analysis of O-acetylated sialic acids in dried blood spots. Analytical Chemistry, 91(4), 2744-2751.

42. Varki, A., \& Gagneux, P. (2012). Multifarious roles of sialic acids in immunity. Annals of the New York Academy of Sciences, 1253(1), 16-36.

43. Almagro-Moreno, S., \& Boyd, E. F. (2009). Insights into the evolution of sialic acid catabolism among bacteria. BMC Evolutionary Biology, 9(1), 118-118.

44. Ngantung, F. A., Miller, P. G., Brushett, F. R., Tang, G. L., \& Wang, D. I. C. (2006). RNA interference of sialidase improves glycoprotein sialic acid content consistency.Biotechnology and Bioengineering, 95(1), 106-119.

45. Schnaar, R. L. (2015). Glycans and glycan-binding proteins in immune regulation: A concise introduction to glycobiology for the allergist. The Journal of Allergy and Clinical Immunology, 135(3), 609-615.

46. Helenius, A., \& Aebi, M. (2001). Intracellular functions of N-linked glycans. Science,291(5512), 2364-2369.

47. Preston, R. J. S., Rawley, O., Gleeson, E. M., \& O'Donnell, J. S. (2013). Elucidating the role of carbohydrate determinants in regulating hemostasis: Insights and opportunities. Blood, 121(19), 3801-3810. 
48. Loureiro, L. R., Sousa, D. P., Ferreira, D., Chai, W., Lima, L., Pereira, C., Videira, P. A. (2018). Novel monoclonal antibody L2A5 specifically targeting sialyl-tn and short glycans terminated by alpha-2-6 sialic acids. Scientific Reports, 8(1), 12196-16.

49. Yang, S., Höti, N., Yang, W., Liu, Y., Chen, L., Li, S., \& Zhang, H. (2017). Simultaneous analyses of N-linked and O-linked glycans of ovarian cancer cells using solid-phase chemoenzymatic method. Clinical Proteomics, 14(1), 3-3.

50. Yu, C., \& Withers, S. G. (2015). Recent developments in enzymatic synthesis of modified sialic acid derivatives. Advanced Synthesis \& Catalysis, 357(8), 1633-1654.

51. Cheng, J., Yu, H., Lau, K., Huang, S., Chokhawala, H. A., Li, Y., Chen, X. (2008). Multifunctionality of campylobacter jejuni sialyltransferase CstII: Characterization of GD3 GT3 oligosaccharide synthase, GD3 oligosaccharide sialidase, and trans-sialidase activities. Glycobiology, 18(9), 686-697.

52. Guérardel, Y., Chang, L., Fujita, A., Coddeville, B., Maes, E., Sato, C., Kitajima, K. (2012). Sialome analysis of the cephalochordate branchiostoma belcheri, a key organism for vertebrate evolution. Glycobiology, 22(4), 479-491.

53. Noel, M., Gilormini, P., Cogez, V., Yamakawa, N., Vicogne, D., Lion, C., HarduinLepers, A. (2017). Probing the CMP-Sialic acid donor specificity of two human $\beta$-d-Galactoside sialyltransferases (ST3Gal I and ST6Gal I) selectively acting on O- and N-Glycosylproteins.Chembiochem, 18(13), 1251-1259.

54. Teppa, R. E., Petit, D., Plechakova, O., Cogez, V., \& Harduin-Lepers, A. (2016). Phylogenetic-Derived Insights into the Evolution of Sialylation in Eukaryotes: Comprehensive Analysis of Vertebrate $\beta$-Galactoside $\alpha 2,3 / 6$ Sialyltransferases (ST3Gal and ST6Gal). International journal of molecular sciences, 17(8), 1286.

55. Bhide, G. P., \& Colley, K. J. (2017). Sialylation of N-glycans: Mechanism, cellular compartmentalization and function. Histochemistry and Cell Biology, 147(2), 149-174.

56. Meng, L., Forouhar, F., Thieker, D., Gao, Z., Ramiah, A., Moniz, H., Moremen, K. W. (2013). Enzymatic basis for N-glycan sialylation: Structure of rat $\alpha 2,6-$ sialyltransferase (ST6GAL1) reveals conserved and unique features for glycan sialylation. The Journal of Biological Chemistry, 288(48), 34680-34698.

57. Lopez Aguilar, A., Meng, L., Hou, X., Li, W., Moremen, K. W., \& Wu, P. (2018). Sialyltransferase-based chemoenzymatic histology for the detection of $\mathrm{N}$ - and O-glycans.Bioconjugate Chemistry, 29(4), 1231-1239. 
58. McCarthy, C., Saldova, R., Wormald, M. R., Rudd, P. M., McElvaney, N. G., \& Reeves, E. P. (2014). The role and importance of glycosylation of acute phase proteins with focus on alpha-1 antitrypsin in acute and chronic inflammatory conditions. Journal of Proteome Research, 13(7), 3131-3143.

59. Lindhout, T., Iqbal, U., Willis, L. M., Reid, A. N., Li, J., Liu, X., ... Wong, C. (2011). Site-specific enzymatic polysialylation of therapeutic proteins using bacterial enzymes.Proceedings of the National Academy of Sciences of the United States of America, 108(18), 7397-7402.

60. Gilormini, P., Lion, C., Noel, M., Krzewinski-Recchi, M., Harduin-Lepers, A., Guérardel, Y., \& Biot, C. (2016). Improved workflow for the efficient preparation of ready to use CMP-activated sialic acids. Glycobiology, 26(11), 1151-1156.

61. Wawrzinek, R., Ziomkowska, J., Heuveling, J., Mertens, M., Herrmann, A., Schneider, E., \& Wessig, P. (2013). DBD dyes as fluorescence lifetime probes to study conformational changes in proteins.Chemistry - A European Journal, 19(51), 17349-17357.

62. Howlett, R., Anttonen, K., Read, N., \& Smith, M. C. M. (2018). Disruption of the GDP-mannose synthesis pathway in streptomyces coelicolor results in antibiotic hyper-susceptible phenotypes. Microbiology (Reading, England), 164(4), 614-624.

63. González-Zamorano, M., Mendoza-Hernández, G., Xolalpa, W., Parada, C., Vallecillo, A. J., Bigi, F., \& Espitia, C. (2009). Mycobacterium tuberculosis glycoproteomics based on ConA-lectin affinity capture of mannosylated proteins. Journal of Proteome Research, 8(2), 721-733.

64. Trubitsyn, I. V., Belousova, E. V., Tutukina, M. N., Merkel, A. Y., Dubinina, G. A., \& Grabovich, M. Y. (2014). Expansion of ability of denitrification within the filamentous colorless sulfur bacteria of the genus thiothrix. FEMS Microbiology Letters, 350, 72-80.

65. Patra, D., Mishra, P., Surolia, A., \& Vijayan, M. (2014). Structure, interactions and evolutionary implications of a domain-swapped lectin dimer from mycobacterium smegmatis.Glycobiology, 24(10), 956-965.

66. Schembri, M. A., Sokurenko, E. V., \& Klemm, P. (2000). Functional flexibility of the FimH adhesin: Insights from a random mutant library. Infection and Immunity, 68(5), 2638-2646. 
67. Lopez Aguilar, A., Briard, J. G., Yang, L., Ovryn, B., Macauley, M. S., \& Wu, P. (2017). Tools for Studying Glycans: Recent Advances in Chemoenzymatic Glycan Labeling. ACS chemical biology, 12(3), 611-621.

68. Barny, A., Zhenjun, D., Oleg, G., Aleshkov, S., Hingorani, R., Edinger, M., Lee, R., Link, J., \& Dubrovsky, T. (2009). 3-Carboxy-6-chloro-7-hydroxycoumarin: A highly fluorescent, water-soluble violet-excitable dye for cell analysis. Analytical Biochemistry, 386(2), 262-269.

69. Xie, L., Chen, Y., Wu, W., Guo, H., Zhao, J., \& Yu, X. (2012). Fluorescent coumarin derivatives with large stokes shift, dual emission and solid state luminescent properties: An experimental and theoretical study. Dyes and Pigments, 92(3), 1361.

70. Jones, G., \& Jimenez, J. A. C. (2001). Azole-linked coumarin dyes as fluorescence probes of domain-forming polymers. Journal of Photochemistry \& Photobiology, B: Biology, 65(1), 5-12.

71. Ernst, L. A., Gupta, R. K., Mujumdar, R. B., \& Waggoner, A. S. (1989). Cyanine dye labeling reagents for sulfhydryl groups. Cytometry, 10(1), 3-10.

72. Atwood, J.L., Gokel, G.W., \& Barbour, L. (2017). Comprehensive Supramolecular Chemistry II. Elsevier Science.

73. Kvach, M. V., Ustinov, A. V., Stepanova, I. A., Malakhov, A. D., Skorobogatyi, M. V., Shmanai, V. V., \& Korshun, V. A. (2008). A convenient synthesis of cyanine dyes: Reagents for the labeling of biomolecules. European Journal of Organic Chemistry, 2008(12), 2107-2117.

74. Ueno, T., Urano, Y., Setsukinai, K., Takakusa, H., Kojima, H., Kikuchi, K., Nagano, T. (2004). Rational principles for modulating fluorescence properties of fluorescein. Journal of the American Chemical Society, 126(43), 14079-14085.

75. Sun, W., Gee, K. R., Klaubert, D. H., \& Haugland, R. P. (1997). Synthesis of fluorinated fluoresceins. The Journal of Organic Chemistry, 62(19), 64696475.

76. Sjöback, R., Nygren, J., \& Kubista, M. (1995). Absorption and fluorescence properties of fluorescein. Spectrochimica Acta Part A: Molecular and Biomolecular Spectroscopy, 51(6), L7-L21.

77. Gök, E., \& Olgaz, S. (2004). Binding of fluorescein isothiocyanate to insulin: A fluorimetric labeling study. Journal of Fluorescence, 14(2), 203-206.

78. Mydock-McGrane, L. K., Hannan, T. J., \& Janetka, J. W. (2017). Rational design strategies for FimH antagonists: New drugs on the horizon for urinary tract 
infection and Crohn's disease. Expert Opinion on Drug Discovery, 12(7), 711731.

79. Sparks, M. A., Williams, K. W., Lukacs, C., Schrell, A., Priebe, G., Spaltenstein, A., \& Whitesides, G. M. (1993). Synthesis of potential inhibitors of hemagglutination by influenza virus: Chemoenzymic preparation of $\mathrm{N}-5$ analogs of $\mathrm{N}$-acetylneuraminic acid. Tetrahedron, 49(1), 1-12. 